\title{
MINIMUM ENERGY BROADCAST IN DUTY CYCLED WIRELESS SENSOR NETWORKS
}

\author{
Mosarrat JAHAN
}

A THESIS

IN

The Department

$\mathrm{OF}$

Computer Science And Software Engineering

Presented in Partial Fulfillment of the Requirements

For the Degree of Master of Computer Science

Concordia University

Montréal, Québec, Canada

APRIL 2012

(C) Mosarrat Jahan, 2012 


\title{
Concordia University
}

\author{
School of Graduate Studies
}

This is to certify that the thesis prepared

By: $\quad$ Mosarrat Jahan

Entitled: $\quad$ Minimum Energy Broadcast in Duty Cycled Wireless Sensor Networks and submitted in partial fulfillment of the requirements for the degree of

\section{Master of Computer Science}

complies with the regulations of this University and meets the accepted standards with respect to originality and quality.

Signed by the final examining commitee:

Chair

Dr. R. Witte

Examiner

Dr. J. Opatrny

Examiner

Dr. T. Fevens

Supervisor

Dr. L. Narayanan

Approved by

Chair of Department or Graduate Program Director

20

Robin A.L. Drew, Ph.D.,ing., Dean

Faculty of Engineering and Computer Science 


\title{
Abstract \\ Minimum Energy Broadcast in Duty Cycled Wireless Sensor Networks
}

\author{
Mosarrat Jahan
}

We study the problem of finding a minimum energy broadcast tree in duty cycled wireless sensor networks. In such networks, every node has a wakeup schedule and is awake and ready to receive packets or transmit in certain time slots during the schedule and asleep during the rest of the schedule. We assume that a forwarding node needs to stay awake to forward a packet to the next hop neighbor until the neighbor is awake. The minimum energy broadcast tree minimizes the number of additional time units that nodes have to stay awake in order to accomplish broadcast. We show that finding the minimum energy broadcast tree is NP-hard. We give two algorithms for finding energy-efficient broadcast trees in such networks. We performed extensive simulations to study the performance of these algorithms and compare them with previously proposed algorithms. Our results show that our algorithms exhibit the best performance in terms of average number of additional time units a node needs to be awake, as well as in terms of the smallest number of highly loaded nodes, while being competitive with previous algorithms in terms of the total number of transmissions and delay. 


\section{Acknowledgments}

First and foremost I would like to express my gratefulness to Almighty Allah to make this thesis possible.

I would like to express my sincere gratitude to my supervisor Dr. Lata Narayanan. Her invaluable guidance and encouragement made my thesis work a wonderful learning experience. It is a great opportunity for me to interact with her that enrich my growth as a student as well as a researcher. Thanks to Dr. Lata for her insightful comments and continuous support for the whole duration of my research work. Her endless valuable suggestions and comments gradually matured my research work.

I wish to thank my colleagues and friends for their continuous encouragement. Special thanks to my friend Shaily Kabir and Rajneesh Kumar and my elder sister Upama Kabir. They always motivate me to keep faith in myself.

Finally I would like to express my deepest gratitude to my beloved parents, A. F. M Shahjahan and Shireen Akter, for their unconditional love, continuous support and having faith in me. Without their encouragement, it is not possible for me to finish the degree. 


\section{Contents}

List of Figures viii

List of Tables $\quad$ xi

1 Introduction 1

1.1 Broadcast Operation in Duty Cycled WSN . . . . . . . . . . . 4

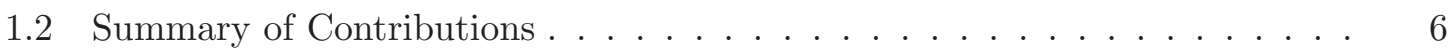

1.3 Outline of Thesis . . . . . . . . . . . . . . . . 7

2 Related Work $\quad 8$

2.1 Broadcasting in $\mathrm{WSN} \ldots \ldots \ldots \ldots \ldots$

2.1.1 Neighbor-Knowledge based Broadcasting . . . . . . . . . . . 9

2.1 .2 Adaptive Broadcasting . . . . . . . . . . . . . . . . . . 10

2.1.3 Probability-Based Broadcasting . . . . . . . . . . . . . 12

2.1.4 Energy Efficient Broadcasting . . . . . . . . . . . . . 13

2.1.5 Multipoint Relay based Broadcasting . . . . . . . . . . . . 17

2.1.6 Connected Dominating Set based Broadcasting . . . . . . . . . . . . 20

2.1.7 RNG and LMST based Broadcasting . . . . . . . . . . . . . 21

2.2 Broadcasting in Duty Cycled WSN . . . . . . . . . . . . . 25

2.2 .1 Centralized Algorithms _. . . . . . . . . . . 25

2.2 .2 Distributed Algorithms _. . . . . . . . . . . . . . . . 31

2.2.3 Differences with Our Work . . . . . . . . . . . . . 35 
3 Algorithms and NP-Completeness $\quad 37$

3.1 Definitions and Preliminaries f . . . . . . . . . . . . . 37

$3.2 \quad$ NP-Completeness of MEBT Problem . . . . . . . . . . . . . . . 39

3.3 Algorithms . . . . . . . . . . . . . . . . . . . . . . 42

3.3.1 Spanning Tree with Incremental Cost (STIC) Algorithm . . . . . . . 43

3.3.2 MST_Edmonds Algorithm . . . . . . . . . . . . . . . 53

3.3.3 The Sweep Operation . . . . . . . . . . . . . . . . . 56

4 Experimental Results $\quad 62$

4.1 Performance Comparison of all Algorithms without Sweep Operation . . . . 63

4.1.1 Average Number of Additional Active Time Units per Node . . . . . 63

4.1 .2 Energy Distribution . . . . . . . . . . . . 66

4.1.3 Number of Node Transmissions . . . . . . . . . . . . . . . . . . 69

4.1.4 Maximum Delay of Broadcast Operation . . . . . . . . . . . 72

4.1.5 Average Delay of Broadcast Operation . . . . . . . . . . . . . 74

4.2 Performance Analysis of Various Versions of STIC Algorithm . . . . . . 77

4.2.1 Average Number of Additional Active Time Units per Node . . . . . 77

4.2 .2 Energy Distribution . . . . . . . . . . . . . 78

4.2 .3 Number of Node Transmissions . . . . . . . . . . . . . . . . 78

4.2.4 Maximum Delay of Broadcast Operation . . . . . . . . . . . 79

4.2.5 Average Delay of Broadcast Operation . . . . . . . . . . . . . 80

4.3 Performance Analysis of Various Versions of MST_Edmonds Algorithm . . . 80

4.3.1 Average Number of Additional Active Time Units per Node . . . . . 81

4.3.2 Energy Distribution . . . . . . . . . . . . . . 81

4.3.3 Number of Node Transmissions . . . . . . . . . . . . . . . . . . . 82

4.3.4 Maximum Delay of Broadcast Operation . . . . . . . . . . . 82

4.3.5 Average Delay of Broadcast Operation . . . . . . . . . . . . . 84

4.4 Summary of Effect of Sweep Operation . . . . . . . . . . . . . 86

4.5 Performance Comparison of all Algorithms with Sweep Operations . . . . . 86 
4.5.1 Average Number of Additional Active Time Units per Node . . . . 87

4.5.2 Distribution of Energy Usage . . . . . . . . . . . . . . . . . 88

4.5.3 Number of Node Transmissions . . . . . . . . . . . . . . . . . . . . . 90

4.5.4 Maximum Delay of Broadcast Operation . . . . . . . . . . . . . . . 91

4.5.5 Average Delay of Broadcast Operation . . . . . . . . . . . . . . . . 92

4.6 Impact of Node Density . . . . . . . . . . . . . . . . . . . 96

4.7 Impact of Number of Nodes . . . . . . . . . . . . . . . . . . . . . 98

4.8 Impact of Schedule Length _ . . . . . . . . . . . . . . . . . . 101

5 Conclusions and Future Work 104

$\begin{array}{ll}\text { Bibliography } & 105\end{array}$ 


\section{List of Figures}

1 Abstract shapes of $C(n)$ and $A(n) \ldots \ldots \ldots \ldots$

2 The edge $(u, v)$ not in $E$ because of $w \ldots \ldots \ldots \ldots \ldots \ldots$

3 Four cases of connecting a $\operatorname{Cov}_{i}(v)$ to the existing $T_{\text {bcast }}$ through $v \ldots \ldots$

$4 \quad G$ for variables $x_{1}, x_{2}, x_{3}$ and $C_{1}=x_{1} \bigvee x_{2} \bigvee \bar{x}_{3}, C_{2}=\bar{x}_{1} \bigvee x_{2} \bigvee x_{3} \ldots \ldots$

$5 \quad$ Broadcast Tree of $G$ with $W=3 \ldots \ldots \ldots \ldots$. . . . . . . . . 41

6 Example of a duty cycled WSN $G$ used to illustrate algorithms . . . . . . 43

$7 \quad$ Construction of $T$ with STIC algorithm with $\operatorname{cost}(T)=15 \ldots \ldots$. . . . 47

8 Construction of $T$ with STIC algorithm with $\operatorname{cost}(T)=15 \ldots \ldots \ldots$

9 Construction of $T$ with STIC algorithm with $\operatorname{cost}(T)=15 \ldots \ldots$. . . . 49

10 Construction of $T$ with STIC algorithm with $\operatorname{cost}(T)=15 \ldots \ldots$. . . 50

11 Construction of $T$ with STIC algorithm with $\operatorname{cost}(T)=15 \ldots \ldots$. . . . 51

12 Construction of $T$ with STIC algorithm with $\operatorname{cost}(T)=15 \ldots \ldots \ldots$

13 Construction of $T$ with MST_Edmonds algorithm with $\operatorname{cost}(T)=11 \ldots$. . . 57

14 Construction of $T$ with MST_Edmonds algorithm with $\operatorname{cost}(T)=11 \ldots 58$

$15 S$ as well as broadcast tree $T$ obtained after extraction phase . . . . . . 59

16 Sweep Operation on $T \ldots \ldots \ldots \ldots \ldots \ldots$. . . . . . . . . . . . . . . . .

17 Average no. of additional active time units per node at node density 8 for all values of sch_len and $N=400 \ldots \ldots \ldots \ldots$. . . . . . . . 63

18 Average no. of additional active time units per node for all values of sch_len




19 Distribution of Energy Usage at node density $=12$ when $N=400$ and sch_len $=$

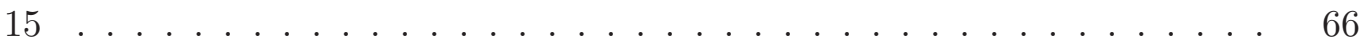

20 Total number of node transmissions at node density 12 for sch_len $=20$. . 70

21 Total number of node transmissions for sch_len $=20 \ldots \ldots \ldots$. . . . 71

22 Maximum Delay of the Network at node density 12 for sch_len $=20 \ldots 72$

23 Maximum Delay of the Network at node density 10 and 8 for sch_len $=20$. 73

24 Average Delay of the Broadcast Opeation at node density 12 for sch_len $=2075$

25 Average Delay of the Network at node density 10 and 8 for sch_len $=20 \ldots 76$

26 Average no. of additional active time units per node for various versions of STIC algorithm at node density $=12$ and $N=400 \ldots \ldots \ldots 77$

27 Distribution of Energy Usage for STIC and STIC_inc algorithms at node

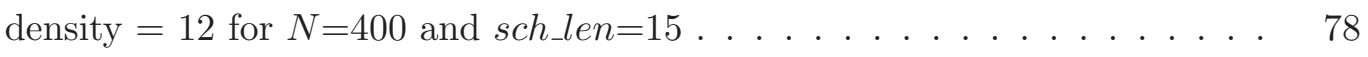

28 Number of node transmissions for STIC and STIC_inc at node density $=12$

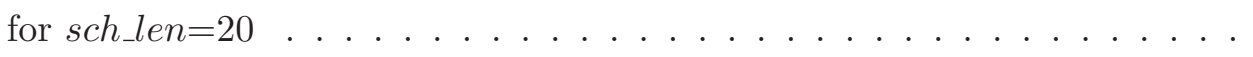

29 Maximum Delay of the Network for STIC and STIC_bfs at node density 12 for $s c h \_l e n=20 \ldots \ldots \ldots \ldots \ldots \ldots \ldots \ldots$

30 Average Delay of the Network for STIC and STIC_bfs at node density 12 for sch_len $=20 \ldots \ldots \ldots \ldots \ldots \ldots \ldots \ldots \ldots \ldots \ldots \ldots \ldots \ldots \ldots \ldots \ldots$

31 Average no. of additional active time units per node for various versions of MST_Edmonds algorithm at node density $=12$ and $N=400 \ldots \ldots$. . .

32 Distribution of Energy Usage for MST_Edmonds and MST_Edmonds_bfs algorithms at node density $=12$ for $N=400$ and sch_len=15 . . . . . . . .

33 Number of node transmissions for MST_Edmonds and MST_Edmonds_bfs at node density $=12$ for $s c h \_l e n=20 \ldots \ldots \ldots \ldots$

34 Maximum Delay of the Network for MST_Edmonds and MST_Edmonds_bfs at node density 12 for $s c h \_l e n=20 \ldots \ldots \ldots \ldots$

35 Average Delay of the Network for MST_Edmonds and MST_Edmonds_bfs at node density 12 for sch_len $=20 \ldots \ldots \ldots \ldots \ldots \ldots$

36 Maximum Delay of the Network at node density 12 for sch_len $=20 \ldots 92$ 
37 Maximum Delay of the Network for sch_len $=20 \ldots \ldots$. . . . . 93

38 Average Delay of the Network at node density 12 for $s c h \_l e n=20 \ldots . . .94$

39 Average Delay of the Network for sch_len $=20 \ldots \ldots \ldots$

40 Average no. of additional active time units per node for various values of $N$ at node density 12 when sch_len=20 . . . . . . . . . . . . . . 97

41 Percentage of nodes with active time units 14 for various values of $N$ at node density 12 and sch_len=15 . . . . . . . . . . . . . . . . 99

42 Average No. of Node Transmissions for various values of $N$ at node density 12 and $s c h \_l e n=20 \ldots \ldots \ldots \ldots \ldots \ldots$

43 Total Active Time Units per Node for STIC_inc in a time period of 60 at

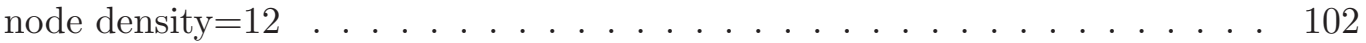

44 Total Active Time Units per Node for MST_Edmonds_bfs in a time period of 60 at node density $=12 \ldots \ldots \ldots \ldots$ 


\section{List of Tables}

1 Average Additional Active Time Units per Node with respect to MST_Edmonds for $N=400$ and $s c h \_l e n=20 \ldots \ldots \ldots \ldots \ldots \ldots$

2 Distribution of Energy Usage at node density 8 when $N=400$ and sch_len=15 67

3 Distribution of Energy Usage at node density 10 when $N=400$ and sch_len $=1568$

4 Distribution of Energy Usage at node density 12 when $N=400$ and sch_len=15 68

$5 \quad$ Percentage of Nodes with active time units 14 with respect to MST_Edmonds when $N=400$ and $s c h \_l e n=15 \ldots \ldots \ldots$. . . . . . . . . . 69

6 Percentage of Nodes with active time units 7 with respect to MST_Edmonds when $N=400$ and $s c h \_l e n=15 \ldots \ldots \ldots \ldots \ldots$

7 Total Number of Node Transmissions with respect to CSCA when $N=400$,

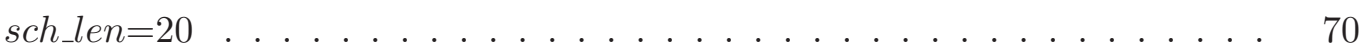

8 Normalized Maximum Delay with respect to SDT when $N=400$ and sch_len=20 74

9 Normalized Average Delay with respect to SDT when $N=400$ and sch_len=20 74

10 Improvements obtained by the sweep operation at node density 12 for $N=400$ : metric for best variant of sweep divided by metric for algorithm without sweep 86

11 Best version of sweep operation for various combinations of broadcast tree algorithm and cost measure . . . . . . . . . . . . . . . 86

12 Average Additional Active Time Units per Node for $N=400$ and sch_len $=2087$

13 Average Additional Active Time Units per Node with respect to MST_Edmonds_bfs for $N=400$ and sch_len $=20 \ldots \ldots \ldots \ldots$. . . . . . . . 87

14 Percentage of Nodes with active time units 14 when $N=400$ and sch_len=15 88 
15 Percentage of Nodes with active time units 7 when $N=400$ and sch_len=15 88

16 Percentage of Nodes with active time units of 14 with respect to MST_Edmonds_bfs when $N=400$ and $s c h \_l e n=15 \ldots \ldots \ldots \ldots$. . . . . . . . . . . . 89

17 Percentage of Nodes with active time units of 7 with respect to MST_Edmonds_bfs when $N=400$ and sch_len=15 . . . . . . . . . . . . . . . . . 89

18 Number of Node Transmissions when $N=400$ and sch_len=20 . . . . . . 90

19 Number of Node Transmissions with respect to CSCA_bfs when $N=400$ and sch $l e n=20 \ldots \ldots \ldots \ldots \ldots \ldots$

20 Normalized Maximum Delay with respect to SDT when $N=400$ and sch_len=20 91

21 Normalized Average Delay with respect to SDT when $N=400$, sch_len=20 . 94 


\section{Chapter 1}

\section{Introduction}

A Wireless Sensor Network (WSN) is a densely populated network consisting of a large number of battery-operated sensor nodes. Sensor nodes are low power devices that usually contain one or more sensors, a processor, memory, a power supply, a radio and an actuator. Such a node utilizes a wide variety of sensors like mechanical, thermal, biological, chemical, optical, magnetic etc. to measure different aspects of the environment [2]. Sensor nodes are equipped with a processor and a limited size memory and thus capable of performing simple calculations. Wireless communication among the nodes is established through the radio. If two nodes are within the transmission range of each other they can communicate directly. Otherwise intermediate nodes between the two end points should forward the packets. The positions of the sensor nodes in a WSN are not usually predetermined and they are deployed in large number to achieve accurate computation and to overcome the limitations imposed by short transmission range of nodes. In most applications of WSNs, a large body of sensor nodes are deployed in an ad hoc manner to monitor certain aspects of the environment and the nodes periodically send data to a base station.

WSNs have huge potential in a wide range of applications such as health, military, home and environment. Due to the rapid deployment, self-organization, and fault tolerance properties, WSNs are very promising in military applications. They can be exploited for military command, control, communication, computing, intelligence, surveillance, reconnaissance and targeting systems [3]. Particularly, in a battlefield a WSN can be utilized 
for surveillance of critical terrains and routes. In a target tracking system, a WSN can be used for detection and identification of intruders. Moreover, a WSN can be exploited for gathering information about battle damage assessment. In the health care setting, sensor nodes can be deployed to monitor the conditions of patients and assist disabled patients. The chance of getting and prescribing the wrong medication to the patients is decreased if sensor nodes are used to administer medications. For home applications, sensor nodes and actuators can be included in appliances like vacuum cleaners, microwave ovens, refrigerators etc. Sensor nodes inside these domestic devices can interact with each other using external networks or the Internet. They allow the end users to control home devices more conveniently either locally or remotely. In environmental applications, WSNs can be exploited for tracking the movements of animals and monitoring environmental conditions affecting crops and livestock. They can be utilized for large-scale earth monitoring, planetary exploration, biological, earth, and environmental monitoring in marine, soil and atmospheric contexts, forest fire detection, pollution studies etc. Some of the commercial applications include managing inventory, monitoring product quality, monitoring material fatigue, environment control in office building and monitoring disaster areas.

Due to the huge prospects for WSNs, significant research has been conducted to devise suitable solutions for various challenges of WSN as well as to adapt the existing protocols of other networks for WSN. Unlike traditional networks, a WSN has its own design issues and resource limitations. Resource constraints include limited energy source, short communication range, low bandwidth and limited processing and storage capacity in each node. Generally in WSNs a large number of sensor nodes work together unattended. Sensor nodes may be deployed in hostile environments such as disaster recovery where it is not possible to replace the battery of the nodes. Thus, the topology of a WSN changes because of the death of nodes due to running out of power as well as because of the addition of new nodes. This in turn indicates that the protocols and algorithms for WSNs should be designed with self-organizing capability. Moreover, the failure of nodes should not affect the overall operation of a WSN; this property is known as fault tolerance. Due to large scale deployment, protocols and algorithms for WSN should be scalable, and resilient to 
changes in topology. Moreover wise utilization of battery power is essential for long time operation of WSN. Malfunctioning of sensor nodes significantly changes the topology and thus necessitates rerouting of data packets and reorganization of the network. Thus, power conservation and power management take on additional importance. A huge amount of research is going on to design power-aware protocols and algorithms for WSNs. Routing in WSNs is more challenging due to their unique characteristics that distinguish them from other wireless networks [4]. Due to large scale deployment of sensor nodes it is not possible to build a global addressing scheme as the overhead of ID maintenance is high. Moreover, sensor nodes deployed in an ad hoc fashion should be self-organizing in order to get itself accustomed in the existing WSN. Routing in WSN is data-centric as there is no global addressing scheme. Due to tight constraints on energy, processing, and storage capacities, routing in WSN requires careful resource management.

Due to extremely limited energy it is not feasible for WSN to operate as networks that are always operational. The fundamental idea of duty cycled WSNs is to reduce the time spent by a node in idle state or overhearing other transmissions by putting the node in sleep state. Each sensor keeps itself active for only a very brief period of time and this is known as active state, while it stays dormant for a long time. During its active state a node can sense an event, transmit a packet or receive a packet, or even stay idle. During the sleep state, a node turns all its functional units off except a timer, to wake itself up after a fixed amount of time. The concept of a duty cycle is represented by a periodic wake up schedule associated with every sensor node. The duty cycle is measured as the ratio of the number of active time units to the total number of time units. It indicates how long a node spends in active state. A small duty cycle signifies that a node is asleep most of the time. The duty cycle of a WSN is determined based on the requirements of the applications for which the network is deployed.

Routing in duty cycled WSN becomes more complicated since a transmitting node may not be active at the same time unit as its neighbors. Thus a node needs to wait until it can forward a message to its neighbors. Unlike other wireless networks where a node can reach all its neighbors with one message, in a duty cycled network, a node may need to transmit 
to all its neighbors separately. Providing an energy efficient communication mechanism for duty cycled WSN is the main focus of this thesis.

\subsection{Broadcast Operation in Duty Cycled WSN}

Broadcasting is a fundamental operation in wireless networks where data transmitted by a node is sent to all nodes in the networks. For example, various reactive or on-demand routing protocols such as AODV, DSR etc utilize the concept of discovering of a route when the actual need to route data arises [14]. The route discovery mechanism depends on the broadcast operation to determine a route between a source and destination nodes. Mobile Ad-hoc Network (MANET) can use delay-efficient broadcast operation to quickly disseminate information such as an link breakage message to the whole network so that the topology information is updated in every node [47]. Many real-time applications such as audio conferencing also require a low-latency broadcast operation to deliver delay-sensitive data over the wireless ad hoc networks.

In WSNs as well as in duty cycled WSNs, broadcast operation has significant impact. In most applications of WSN, a large number of sensor nodes monitor the occurrence of an event and inform a central node known as the sink. Conversely, the sink node broadcasts control messages during network configuration time and interest/ query messages at the time of data acquisition. Broadcast operation is one of the basic communication services in WSN that is used to establish communication between sink node and other sensor nodes. Non-sink sensor nodes may also broadcast messages in order to synchronize with other nodes to monitor certain events [46]. Various routing protocols for WSN also use broadcast operation as the integral part of their operation. For example, in LEACH (Low-Energy Adaptive Clustering Hierarchy) [15], a node selects itself as a cluster head and informs all the nodes in the network through broadcasting an advertisement message. Various data centric routing protocols also utilize broadcast operation for the purpose of data collection. For example, in directed diffusion [21], the sink node requests data by broadcasting interest messages. In SPIN (Sensor Protocols for Information via Negotiation) [16], when a node 
has new data to share, it broadcasts an advertisement message to all nodes in the network about the new data.

Multicasting is an important mechanism of communication used for sending information to a group of nodes. Many researchers utilize the mechanism of broadcast operation to determine a suitable solution for multicasting. They first construct a broadcast tree that will cover all the nodes in the networks and then eliminate the transmissions that are not directed to the members of the multicast group. This application of broadcast tree is used in [49], [51].

In the literature most research has been aimed on minimizing the number of transmissions in a broadcast operation while achieving whole network coverage as well as providing reliable broadcast operation by minimizing collisions. Moreover some work has been motivated by the need to minimize the delay of the broadcast operation.

Designing energy-efficient broadcast algorithm for duty cycled WSNs is a challenging problem as sensor nodes switch between active and sleep modes. Clearly the energy consumption during sleep mode is much less than that in any other mode. However, going into sleep mode is not without cost. In fact, there is a significant amount of energy as well as time required to change from sleep mode back to transmit mode. To get an idea of switching cost we consider an example mentioned in [24]. When a node wakes up it listens the channel for a brief period of time and measures the signal strength. If it is greater than a threshold value, the node remains active to receive transmission. Otherwise, it will go to sleep. The procedure is known as sniffing the channel [24]. For Chipcon CC1100 radio, if a node wakes up once every second the average current consumption over 1 second is $15 \mu \mathrm{A}$ that will cause a charge draw of $15 \mu \mathrm{C}$. Whereas, the average current draw is $15 \mathrm{~mA}$ for receiving or transmitting a packet. Thus, in a day of operation of the network, the total energy consumption of a node due to wakeup is equal to $15 \mu \mathrm{A} * 3 V^{*} 86400 \mathrm{~s}=3.9 \mathrm{~J}$ that can be utilized to transmit or receive almost 21 Mbits of data. Recently several papers have pointed out that neglecting the so-called switching energy to switch from one mode to another can lead to algorithms with sub-optimal energy consumption or reduce network lifetime [5, 9, 23, 24]. Ruzzelli et al. [38] report measurements on three different chipsets for 
sensor nodes that show that at low traffic load, the switching energy can dominate the energy required for transmission. Thus, depending on the traffic conditions, it is not beneficial to switch to sleep mode at every opportunity.

When a node has a packet to transmit it has two options. As the active time slots of its neighbors are not necessarily synchronized either with the node itself or with each other, one option is for the node to go into sleep mode and wake up and transmit the packet when its first neighbor wakes up, and thereafter repeatedly switch between sleep and transmit modes until it has delivered the packet to all the relevant neighbors. Indeed several papers make this assumption in analyzing the energy costs of their algorithms $[18,46]$. However, as mentioned earlier, this model ignores the high switching cost of switching from sleep to transmit mode. Another option as assumed in [43], is for the node to stay awake until it has delivered the packet to next-hop neighbors. This option not only has the merit of simplicity, it is clearly more energy-efficient when the switching cost is high, when there are many neighbors, or not many slots in between the active times of different neighbors. This is the model we assume in this thesis.

In this thesis, we address the energy inefficiency issue of the broadcast operation in duty cycled WSNs and propose algorithms to minimize the total number of additional active time units the nodes of a network need to be active during the broadcast operation. Given the number of nodes $N$ in a duty cycled WSN and a wakeup schedule of fixed length $k$ for every node, these algorithms construct a broadcast tree that will minimize the total number of additional active time units nodes need to be awake. This problem is known as the Minimum Energy Broadcast Tree (MEBT) problem.

\subsection{Summary of Contributions}

In this thesis we address the MEBT problem for duty cycled WSNs. In this section, we give a summary of our results.

1. We show that the MEBT problem is NP-hard. 
2. We propose two polynomial time algorithms to construct an energy-efficient broadcast tree for MEBT problem: Spanning Tree with Incremental Cost (STIC) and MST_Edmonds.

3. We describe several variants of a sweep operation similar to the one in [51], that can be applied to a given broadcast tree to reduce its cost. Our experimental results show that this operation substantially reduces the cost of the broadcast tree, and generally also improves the performance according to other cost measures.

4. We evaluate the performance of the algorithms using extensive simulations and compare the results with two existing algorithms named Centralized Set Cover based Approximation Algorithm (CSCA) [18] and Shortest_Delay_Tree(SDT) which is adapted from One-to-All Broadcast Algorithm (OTAB) [22]. The experiments show that both MST_Edmonds and STIC have the best performance for the MEBT problem, and they also result in fewer heavily loaded nodes as compared to the two existing algorithms. Their performance is also competitive in terms of the number of node transmissions and maximum and average delay.

\subsection{Outline of Thesis}

In Chapter 2, we present a literature review on broadcast problem in WSNs as well as in duty cycled WSNs. In Chapter 3, we propose our algorithms for energy efficient broadcast tree construction and illustrate the operation of the algorithms with a suitable example.

We analyze the performance of the proposed algorithms in Chapter 4. Some concluding remarks and possible directions for future work are given in Chapter 5. 


\section{Chapter 2}

\section{Related Work}

In this chapter we review the broadcast algorithms for wireless networks that exist in the literature. We will discuss several important broadcast algorithms and try to give an idea of the various trends of the research in this field. We first highlight various algorithms for broadcasting in wireless sensor networks followed by a discussion about the broadcast protocols in duty cycled wireless sensor networks.

\section{$2.1 \quad$ Broadcasting in WSN}

Broadcasting is an important communication paradigm in all networks including wireless sensor networks. The simplest way to broadcast a packet is flooding. In this technique, every node retransmits a packet once when it receives the packet for the first time. It is a very simple technique and ensures that every node receives the packet. The disadvantage of flooding is that it generates abundant retransmissions causing the wastage of battery energy and bandwidth. Retransmissions by geographically close nodes result in message collisions and channel contentions. This scenario is known as the broadcast storm problem [32].

Extensive research has been conducted to reduce the number of retransmissions during the broadcast operation. This optimization leads to the design of energy efficient broadcast protocols that are a necessity for energy-constrained wireless networks. Research is also conducted to build up protocols that will achieve reachability as well as latency-optimized 
operation.

To organize the discussion of protocols, we divide them into a number of groups depending on a number of aspects. Algorithms belonging to the same group have some common characteristics. In the following subsections we will describe the algorithms from various categories.

\subsubsection{Neighbor-Knowledge based Broadcasting}

Algorithms in this category are mainly inspired by the work of H. Lim and C. Kim [29]. They proposed two flooding heuristics named Self-Pruning (SP) and Dominant-Pruning (DP). SP utilizes the direct neighbor information. Node $v$ piggybacks $N(v)$ in the broadcast packet. Another node $u$ receiving the packet, checks whether $N(u)-N(v)-\{v\}$ is empty. If it is empty, $u$ does not forward the packets as all its adjacent nodes already received the packet. The time complexity of the SP is $O(\Delta)$, where $\Delta$ is the maximum degree of the tree.

A similar algorithm to SP is proposed by Peng et el. [34]. They utilize the local topology information and the statistical information about duplicate messages to eliminate unnecessary transmissions. Every node has the knowledge of its 2-hop neighborhood. When a node $u$ receives a message $m$ from a node $v$ it records $N(v) \cap\{v\}$ in its broadcast cover set $C(u, m)$. Then it checks whether $N(u) \subseteq N(v) \cap\{v\}$ and if it is true, then node $u$ avoids transmission of $m$. Otherwise, if message $m$ is received for the first time, $u$ initializes $C(u, m)$ to $N(v) \cap\{v\}$ and waits for a random delay period. During this time node $u$ records $N(v) \cap\{v\}$ in $C(u, m)$ for any $v$ from which it receives a duplicate of $m$. When the delay period is expired, if $N(u) \subseteq C(u, m)$, then $u$ avoids the rebroadcast of $m$. Otherwise $u$ will rebroadcast the message $m$. The delay period is selected carefully so that a node with more neighbors broadcasts earlier as compared to other nodes.

DP uses the 2-hop neighborhood information. The sending node selects from its adjacent nodes a set of forwarding nodes to relay the broadcast packet and appends the IDs of the selected nodes in the broadcast packet which is known as the forward list. A node in the forward list in turn selects the forwarding nodes from its 1-hop neighbors. This process is continued until the broadcast operation is completed. On receiving a packet 
from node $u$ with $v$ in the forward list, node $v$ determines its forward list so that all nodes within 2-hop distance from $v$ receive the packet. Node $v$ tries to cover all nodes in $U=N(N(v))-N(u)-N(v)$, where $N(u)$ and $N(v)$ are the 1-hop neighbor list of $u$ and $v$, respectively. As nodes in $N(u)$ have already received the packet and those in $N(v)$ will receive the packet when $v$ will forward the packet, this algorithm selects a set of forwarding nodes $F=\left\{f_{1}, f_{2}, \ldots, f_{m}\right\}$ from $B(u, v)=N(v)-N(u)$ such that $\bigcup_{f_{i} \in F}\left(N\left(f_{i}\right) \cap U\right)=U$. This algorithm repeatedly selects $v_{k} \in B(u, v)$ which can cover the maximum number of uncovered neighbor nodes. Both SP and DP outperform blind flooding by reducing the redundant retransmissions, while DP achieves the best result. DP obtains this result at the cost of larger overhead of passing the forward list in the broadcast packet. This overhead increases as the host mobility increases.

The authors of [30] identified the deficiencies of DP and proposed two algorithms that reduce the forwarding set further by more effectively utilizing the 2-hop neighborhood information. In Total Dominant Pruning (TDP), $N(N(u))$ is piggybacked in the broadcast packet from $u$. When another node $v$ receives the packet, the 2-hop neighbor set that needs to be covered by the forward list $F$ of $v$ is reduced to $U=N(N(v))-N(N(u))$. As the size of $U$ is reduced, the size of $F$ also gets reduced. The TDP algorithm consumes more bandwidth as the 2-hop neighborhood information of each sender is piggybacked in the broadcast packet. Partial Dominant Pruning (PDP) does not piggyback any neighborhood information with the broadcast packet as in TDP but reduces nodes from $U$ by excluding $P=N(N(u) \cap N(v))$. Thus $U$ will become $N(N(v))-N(u)-N(v)-P$. The extra cost of the PDP algorithm is that each forward node $v$ needs to calculate $P$.

Simulation results show that both TDP and PDP significantly reduce the number of forwarding nodes as compared to DP. TDP produces slightly better result than PDP and PDP is cost effective since there is no piggybacking as in TDP and DP.

\subsubsection{Adaptive Broadcasting}

To alleviate the broadcast storm problem of simple flooding, several threshold-based broadcasting techniques are proposed. The author of [32] proposed a counter-based scheme as 
well as a location-based scheme for broadcast. In the counter-based scheme [32], every host maintains a counter $c$ for each packet. This counter $c$ is used to keep a record of the number of times a host has received a broadcast packet. When $c$ reaches a predefined threshold value $C$, the host refrains from rebroadcasting the packet as the additional coverage achieved through this transmission is very low. In the location-based scheme [32], each host is assumed to be equipped with a positioning device such as GPS. A receiver can accurately calculate the additional coverage that can be achieved from the location of the source from which it heard the broadcast packet. The receiving host uses a predefined threshold $A$ to determine whether it should rebroadcast or not. The location based scheme achieved better performance in terms of both reachability and the amount of savings as compared to the counter-based scheme as more accurate information is used.

The authors of [44] proposed improvements to both the counter-based and the locationbased schemes. Adaptive Counter-Based scheme [44], dynamically adjusts the threshold value $C(n)$ based on local neighbor information and introduces a time delay before broadcasting a packet to reduce the number of redundant transmissions further. A small value of $C(n)$ can significantly reduce the number of redundancies in a dense network while achieving a better reachability. For sparse networks, greater values of $C(n)$ should be used to achieve reachability, which will increase the number of rebroadcasts. Based on the above observations, the authors proposed abstract shapes of $C(n)$ (shown in Figure 1). The adaptive location-based scheme [44], dynamically adjusts the threshold value $A(n)$ based on neighbor information. The authors presented an abstract shape of threshold function $A(n)$ following the same observations for counter-based scheme. As shown in Figure 1, when $n<n_{1}, A(n)$ should be 0 to enforce a host to rebroadcast. Between $n_{1}$ and $n_{2}, A(n)$ gradually increases to balance savings and reachability. After $n>n_{2}, A(n)=0.187$ is used which is the expected additional coverage achieved after a host receives same broadcast packet twice. 


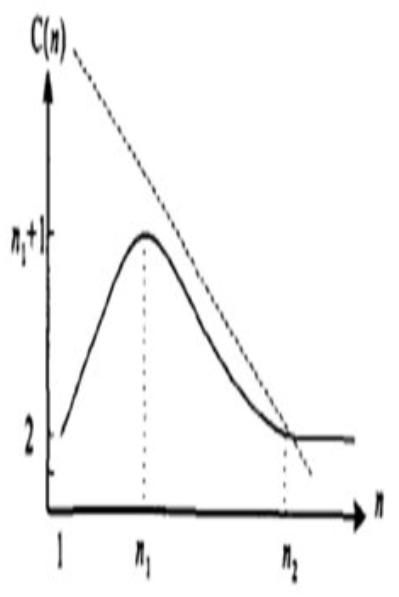

(a) Adaptive Counter-Based Scheme

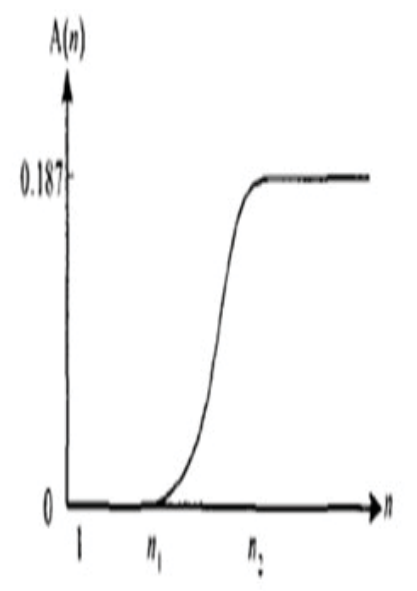

(b) Adaptive Location-Based Scheme

Figure 1: Abstract shapes of $C(n)$ and $A(n)$

\subsubsection{Probability-Based Broadcasting}

In probability-based broadcasting, every node broadcasts with a fixed probability known as gossip probability. In a static gossip strategy, every sensor broadcasts with a fixed probability and this gossip probability is determined during the deployment stage of sensor nodes. The static gossip strategy is not suitable for sensor networks because the topology of the network is not known during the deployment stage. Over-estimation of gossip probability will cause unnecessary packet transmissions in dense networks, while under-estimation causes some portion of the network to be prohibited from getting broadcast packets. Moreover node densities may vary in the same network and the network topology changes by addition of new sensor nodes and deletion of energy-exhausted nodes. The authors of [13] proposed an adaptive gossip protocol known as the adaptive neighbor approach. Here a node chooses its gossip probability in inverse proportion to the number of neighbors it has. The authors of [26] proposed another approach to determine the gossip probability. In this approach, a node chooses its gossip probability for a message with sequence number $k$, in 
inverse proportion to the number of duplicate messages that were overheard for message $k-1$.

Kyasanur et al. [25] identified the deficiencies of the existing adaptive approaches and proposed the smart gossip protocol for wireless sensor networks. In Smart Gossip, the importance of each node $v$ is quantified according to the number of nodes depend on $v$ to receive a disseminated message. When a large number of nodes depends on $v$, it will transmit with higher probability while other less crucial nodes transmit with lower probability. This protocol is completely decentralized and capable of handling wireless link failures and node failures. This protocol significantly reduces energy expenditure by reducing the number of forwarding nodes while achieving the reliability requirements of the application.

\subsubsection{Energy Efficient Broadcasting}

Every node $v$ in a wireless network is associated with a power level $P_{v}$ such that $1 \leq P_{v} \leq$ $m, P_{v} \in Z$. Node $v$ can select its own power level to reach its neighbors. Algorithms in this category try to construct broadcast trees in order to minimize the total power expenditure to accomplish the broadcast operation. W. Liang proved in [28] that the problem of assigning power levels to minimize the total power expenditure is NP-Complete.

Wieselthier et al. [49] proposed three heuristic algorithms for constructing broadcast trees. Broadcast Incremental Power (BIP) algorithm takes advantage of broadcast nature of the wireless channel. This algorithm assumes that the locations of the nodes are fixed. The power needed to maintain the link between node $i$ and $j$ is denoted by $P_{i, j}=r_{i, j}$, where $r_{i, j}$ is the distance between node $i$ and $j$. If a node $i$ is transmitting to its neighbors $j$ and $k$ with transmission power $P_{i, j}$ and $P_{i, k}$ respectively, then a single transmission at power $P_{i,\{j, k\}}=\max \left\{P_{i, j}, P_{i . k}\right\}$ is sufficient to reach both node $j$ and $k$. This property is known as wireless multicast advantage (WMA). BIP starts with a source $s$ and adds a node that can be reached from $s$ with minimum power. For all nodes $i \in T$ and for all adjacent nodes $j$ of $i \notin T$, BIP evaluates the following equation:

$$
P_{i, j^{\prime}}=P_{i, j}-P(i)
$$


where,

$P_{i, j}=$ Cost of transmission between node $i$ and $j$.

$P(i)=$ Power level at which $i$ is already transmitting. If $i$ is a leaf node, then $P(i)=0$.

$P_{i, j^{\prime}}=$ Incremental cost of $i$ to associate $j$ with $i$.

At every step, BIP selects a $j$ with minimum $P_{i, j^{\prime}}$ to be added to $T$ and adjusts the cost $P_{i, k^{\prime}}$ of edges between $i$ and $k$, where $k$ is a neighbor of $i$ not in $T$. This process continues until all nodes are included in T. BIP is similar to Prim's algorithm with only difference is that BIP will dynamically update the cost at each step.

In Broadcast least-Unicast-Cost (BLU) algorithm [49], minimum cost paths from $s$ to every other node are determined and a broadcast tree is obtained by superimposing these unicast paths. As BLU cannot take the advantage of WMA, it produces trees with higher overall power expenditure.

The Broadcast Link-based MST(BLIMST) algorithm [49] associates link cost $P_{i, j}$ with each pair of nodes $i$ and $j$. A minimum cost spanning tree is formed using standard MST techniques. This algorithm also does not take the advantage of WMA.

Wieselthier et al. [49] also found that by rearranging the structure of the broadcast tree significant reduction in overall power expenditure can be achieved. They proposed a operation known as sweep. Given a broadcast tree, the sweep operation makes node $v$ a child of $u$ instead of its previous parent $w$, if doing so reduces the power expenditure at $w$ without increasing the power expenditure at $u$.

The authors of [33] proposed an algorithm to maximize the network lifetime followed by a broadcast operation. Given a sequence of broadcast operations, they tried to increase the number of successful communications before the first communication fails. For this purpose, they proposed an $O(m \log m)$ algorithm to construct a broadcast tree that maximizes the critical energy of the network following a broadcast operation, where $m$ denotes the number of links in the network. The critical energy of a broadcast tree $T$ is the minimum of the remaining battery power of all the nodes in $T$ followed by a broadcast operation. In $T$, the residual energy of node $i$ is $\operatorname{re}(i, T)=c e(i)-\max \{w(i, j) \mid j$ is a child of $i$ in $T\}$, where $c e(i)$ is the current energy of $i$ before sending a message and $w(i, j)$ is the energy 
expended for transmitting a message from $i$ to $j$. The critical energy $C E(T)$ following a broadcast operation is $C E(T)=\min \{r e(i, T) \mid 1 \leq i \leq n\}$. The Maximum Critical Energy Problem (MCEP), finds a broadcast tree $T$ rooted at $s$ such that $C E(T)$ is maximum. This maximum value of $C E(T)$ is called the maximum critical energy and is denoted $M C E(G$, s). This algorithm first constructs a sorted list $L$ of all possible residual energy values. For each node $i$ of $G$, the set $a(i)$ of residual energy values is defined as $a(i)=\{c e(i)-w(i, j) \mid$ $(i, j)$ is an edge of $G$ and $c e(i) \geq w(i, j)\}$. Set $l(i)$ denotes the set of all possible values for residual energy of $i$ following a broadcast operation.

$$
l(i)=\left\{\begin{aligned}
a(i) & \text { if } i=s \\
a(i) \cup\{c(i)\} & \text { otherwise }
\end{aligned}\right.
$$

Thus, $L=\operatorname{sort}\left(\bigcup_{1 \leq i \leq n} l(i)\right)$. The algorithm performs binary search on $L$ to determine $\operatorname{MCE}(G, s)$. For each value $q \in L$, it determines whether there exists a broadcast tree rooted at $s$ such that $C E(T)>q$, by performing breadth first or depth first search that avoids edges $(i, j)$ for which $c e(i)-w(i, j)<q$.

Chen et al. [8] proposed Power Adaptive Broadcasting (PAB) to adjust the transmission power of a node based on its neighbor information. This information is obtained by exchanging the HELLO messages. Each HELLO message contains a list of 1-hop neighbors of a node with the transmission power needed to reach them. In PAB, every node $u$ starts with the most distant node $v$ that causes $u$ to transmit at maximum power level $P_{\max }=P_{u, v}$. Node $u$ determines the subset of its 1-hop neighbors that can reach $v$ and selects a neighbor $w$ that can reach $v$ with minimum transmission power $P_{w, v}$. Node $u$ calculates $P_{u, w}+P_{w, v}$ and if $P_{u, w}+P_{w, v}<P_{u, v}$ it reduces its power level to a lower value and allows $w$ to reach $v$. This is known as local optimization. If $u$ cannot find such a node it transmits using power $P_{u, v}$. After reducing the transmission power level node $u$ starts with the next furthest node and tries to reduce its transmission radius by allowing other neighbor to reach the distant node. This process stops when $u$ cannot find such a neighbor. It may happen that when a node receives a broadcast packet and calculates the local optimization it may considers neighbor that already received the packet. To minimize this problem, after receiving a 
packet, a node may wait for a randomly selected time period. During this time, if it finds that some of its neighbors broadcast the same packet it eliminates these neighbors as well as the nodes that receive the packet from the consideration of local optimization.

$\mathrm{PAB}$ will consume at most the energy consumed in a non-power- adaptive scheme in which the nodes transmit at maximum power level and it achieves the same coverage as nonadaptive schemes. Experimental results show that PAB reduces total energy consumption about $40 \%$ as compared to the protocols that do not adapt the power level.

Weishelthier et el. [50] proposed two distributed versions of the centralized BIP algorithm named as Distributed-BIP-All (Dist-BIP-A) and Distributed-BIP-Gateways (Dist-BIP-G). In Dist-BIP-A, every node $u$ knows the cost of the links between node $u$ and its 1-hop neighbors and also cost of the links between every pair of node $u$ 's 1-hop neighbors. When node $u$ has a broadcast packet, it constructs a local BIP tree using this information and broadcasts this tree to all its neighbors. When a node $v$ becomes aware that it is in the tree from some node $u$, it generates its local BIP tree and broadcasts to the neighbors. A node $v$ can hear from multiple parents but it becomes child of a node from which it hears for the first time. Dist-BIP-All generates huge burden on MAC layer as every node performs the broadcast operation. In Dist-BIP-G, every node $u$ knows the cost of the links between $u$ ' neighbors and their neighbors. Node $u$ has no knowledge of the cost of the links between its 2-hop neighbors $v$ and $w$. Node $u$ constructs local BIP tree using this information and determines the gateway nodes from its 1-hop neighbors that cover one or more nodes in its 2-hop. The set of gateway nodes of $u$ covers all the 2-hop neighbors of $u$. After the construction of BIP tree, node $u$ broadcasts this information to all its neighbors. The links between the gateways and their neighbors are not included in the global tree. Now only the gateway nodes of $u$ will construct their local BIP tree and broadcast this information. It reduces the overhead on MAC layer as only fewer node will broadcast the BIP tree.

A localized version of BIP algorithm (LBIP) is proposed in [19]. In this method, each node constructs a BIP tree within its 2-hop neighborhood using information provided by the node from which it gets the broadcast message. Thus the tree is incrementally constructed. The source node determines the BIP tree within its 2-hop neighborhood and selects the 
nodes within its range that should relay the packet with which transmission radius. These choices are forwarded with the broadcast packet. No instructions are given in the packets for nodes that are designated as leaf nodes. When a node $u$ receives the packet for the first time from a node $v$, two cases can occur:

1. The packet contains some instructions for $u$. It starts constructing a BIP tree within its own 2-hop neighborhood. But instead of starting with an empty tree, it uses the information contained in the packet, that is with the neighbors assigned to it by $v$ and with its transmission range also fixed by $v$. In this way, nodes located exactly at 2 hops from $u$ and 3 hops from $v$ will be added to the tree.

2. There is no instruction for $u$. In this case, $u$ will not rebroadcast the packet.

As the algorithm is localized one, it is possible that two different nodes may make conflicting decisions that will lead to some nodes being uncovered. To avoid this situation, when a node receives a broadcast packet, it will monitor its neighborhood for a fixed amount of time. If the node finds that some of its neighbors do not get the packet, it can transmit the packet to them whether it is instructed to do so or not. This ensures coverage at the cost of some unnecessary transmissions. To minimize these unnecessary transmissions, the set of monitored neighbors can be reduced to a smaller subset of neighbors using a subgraph of the general graph such as RNG or LMST [19]. LBIP eliminates the overhead of message exchange in distributed BIP and does not increase the size of the message significantly. Experimental results showed that this algorithm has good performance at low density and it is very energy efficient for higher densities with performance equal to BIP.

\subsubsection{Multipoint Relay based Broadcasting}

The authors of [36] proposed the mechanism to calculate multipoint relay (MPR) set. This technique reduces the number of redundant message transmissions in broadcast operation. The authors proved that the computation of $M P R$ set with minimum size is NP-Complete

and proposed a heuristic technique to compute the $M P R$ set. Each node calculates it own $M P R$ set independently and modifies its $M P R$ set according to the changes in local 
topology. Every node $u$ starts with an empty $M P R(u)$ set and selects those nodes $v$ from $N(u)$ that are only neighbors of some 2-hop neighbors of $u$. Node $u$ is called the MPR selector of $v$. If there are some 2-hop neighbors that are not covered by $M P R(u)$, a node from $N(u)$ that covers the largest number of 2-hop uncovered nodes and is not already in $M P R(u)$, is selected. This procedure is repeated until there is no uncovered node in the 2-hop neighborhood of $u$. This heuristic gives a result that is within a factor of $\log n$ from optimality, where $n$ is the maximum degree of a node. A forwarding node may or may not actually retransmit a message and its status is determined by a MPR rule:

- A node retransmits a message once if it received the message for the first time from a selector.

The collection of nodes that retransmit the message plus the source node form a connected dominating set $(C D S)$.

The MPR set calculated according to [36] is source-dependent as the forward node set is determined during the broadcast operation and it is dependent on the source of the broadcast and communication latency.

An efficient protocol for broadcasting in Mobile ad hoc networks known as Ad Hoc Broadcast Protocol(AHBP) is proposed in [48]. It is a distributed protocol that utilizes 2-hop topology information of a node to determine broadcast relay gateway (BRG) from its 1-hop neighbors. The set of selected BRG forms a connected dominating set. This way AHBP reduces the number of redundant messages as compared to the flooding protocol. In AHBP, every node maintains a duplicate table and a 2-hop neighbor table. Whenever a node receives a new packet, it makes an entry in the duplicate table and uses this table to drop already received packets. A node uses HELLO message to construct its 2-hop neighbor table. When a node broadcasts a packet, it selects some of its 1-hop neighbors as BRGs and this list is included in the broadcast packet. A broadcast packet also contains information about the route $P$ that the packet already traversed. Only the nodes in the BRG set will rebroadcast the packet. Unlike other protocols, in AHBP, BRGs are calculated on-demand and no virtual backbone structure needs to be maintained. BRGs are picked out along with 
the propagation of broadcast messages. Every node $v$ uses the information $P$ of the route the packet already traversed to eliminates some nodes $w \in P$ as well as its neighbors from the consideration of BRGs. Node $v$ then utilizes its 2-hop neighbor list to select its BRG set in the same way as MPR set is calculated in in [36]. Technique to handle the node mobility is also incorporated in this protocol. Although this protocol is designed for MANET, it can be also utilized in static wireless sensor networks.

Adjih et al. [1] proposed a novel source-independent $M P R$ where the forward node set is determined before any broadcast operation and is constructed based on MPR using two simple rules. It requires the knowledge of total order of the nodes. A node decides to include itself in $C D S$ if and only if:

1. It has the smallest ID among its neighbors OR

2. It is a multipoint relay of its neighbor with smallest ID.

Adjih et al. [1] also proposed two heuristic algorithms to calculate the multipoint relay set. In Min-ID MPR set computation, every node $u$ starts with an empty $M P R(u)$ set and scans its neighbors in the increasing order of their node ID. If the current node covers a 2-hop neighbor that is not covered with the existing $M P R(u)$, then it is added in $M P R(u)$. In reverse $M P R$ selection algorithm, every node $u$ starts with empty $M P R$ selector set. For each pair of neighbors $v$ and $w$, this algorithm determines the nodes that are neighbors of both $v$ and $w$ and if $u$ has the smallest ID among them, then both $v$ and $w$ are added into the $M P R$ selector set of $u$.

$\mathrm{Wu}$ [37] identified two drawbacks for $M P R$ proposed in [1]:

1. Rule 1 is useless in many occasions

2. The Original MPR Forward node selection does not take advantage of Rule 2 .

Based on the observation rule 1 is modified as follows:

1. Enhanced Rule 1: The node has a smaller ID than all its neighbors and it has two unconnected neighbors. 
Node $u$ is called a free neighbor of $v$ if $v$ is not the smallest ID neighbor of $u$. In enhanced forward node selection, all free nodes are included first. A node $u$ in 1-hop neighborhood is added if it is the only neighbor of a 2-hop neighbor. Then a 1-hop node with largest number of uncovered 2-hop nodes is added in MPR set. Node IDs are used to break ties. Simulation results show that the Enhanced $M P R$ (EMPR) with enhanced rule 1 and enhanced forward node set selection reduces the forward node set $10 \%$ as compared to one in [1].

Wu et al. [53] provided several extensions of EMPR [37] to generate a smaller CDS using complete 2-hop neighborhood information. Source-dependent MPR [36], sourceindependent MPR [1] and Enhanced MPR [37] use partial 2-hop neighborhood information to determine their MPR set. Partial 2-hop neighborhood information excludes the links among the 2-hop neighbors. The Complete 2-hop information is obtained after exchanging two rounds of HELLO messages and if the positional information is available. It can be obtained from 3 rounds of message exchange if positional information is not available. In the proposed technique node $v$ repeatedly selects a node pair $(u, w)$ where $u \in H_{1}(v)$ and $w \in H_{1}(u) \cap H_{2}(v)$ until all 2-hop neighbors are covered. $H_{1}(v)$ is the set of nodes that are 1-hop away from $v$ and $H_{2}(v)$ is a set of nodes that are exactly 2-hop away from $v$. Node $u$ is directly covered by $v$, whereas $w$ is indirectly covered by $v$. Node $v$ is called a direct selector of $u$ and an indirect selector of $w$. The authors modified the rule $2[1,37]$ of CDS calculation as follows:

- Enhanced Rule 2: Node $u$ is a forward node if it is directly selected by a node in $H_{1}(u)$ that has smallest ID in $H_{1}(u)$ and $w$ is a forward node if it is indirectly selected by a node in $H_{2}(w)$ that has a smaller ID than all nodes in $H_{1}(w)$.

The set of forward nodes selected by Enhanced Rule 1 and Enhanced Rule 2 form a $C D S$.

\subsubsection{Connected Dominating Set based Broadcasting}

Algorithms in this category construct a connected dominating set (CDS) to perform the task

of broadcasting. Nodes in the CDS are responsible to retransmit the messages. By reducing the size of the CDS, significant improvements can be achieved in number of redundant 
retransmissions. Stojmenovic et al. [42] proposed a dominating set based broadcasting algorithm that computes set of internal nodes with locally available information. This algorithm achieves reliability while significantly reducing the number of retransmissions. A neighbor elimination scheme is used to avoid unnecessary retransmissions. The proposed algorithm for dominating set eliminates the overhead of exchanging information at the time of constructing the dominated set. For this purpose, they modified the algorithm to compute the dominating set provided in [52] and obtain a new algorithm [41]. Here node ID is replaced with key $=($ degree $, x, y)$ where degree is the number of neighbors of a node and $x$ and $y$ represent the $x$ co-ordinate and $y$ co-ordinate of the node, respectively. Thus, nodes with higher degree have a higher chance of becoming internal nodes. A node $u$ is called an intermediate node if it has two unconnected neighbors. If node $u$ is a neighbor of $v$ and if each neighbor of $u$ is also a neighbor of $v$ and $k e y(u)<k e y(v)$, then $u$ is covered by $v$. A node $w$ that is not covered by any neighboring node is called an intergateway node. A node $u$ is covered by two connected neighboring nodes $v$ and $w$ if each neighbor of $u$ is also a neighbor of either $v$ and $w$ and $\operatorname{key}(u)<\operatorname{key}(v)$ and $\operatorname{key}(u)<\operatorname{key}(w)$. A node $x$ not covered by any pair of connected neighboring nodes is called a gateway node. Now a node $u$ can decide whether it belongs to a dominating set or not with its locally available information. The number of retransmissions in a broadcast operation depends on the size of the dominating set. The authors also proposed to use the neighbor elimination technique during the broadcast operation. Here every node $u$ rebroadcasts the message when it receives it for the first time if the set of neighboring nodes of $u$ not receiving the broadcast packet is non-empty. Internal node and neighbor elimination scheme require each node to know the exact location of the neighbors (if GPS is available) or to know the list of neighbors for each of its neighbors.

\subsubsection{RNG and LMST based Broadcasting}

Broadcast protocols in this category address the problem of adjusting transmission power of nodes in order to reduce the total energy consumption of broadcast operations. Broadcast 


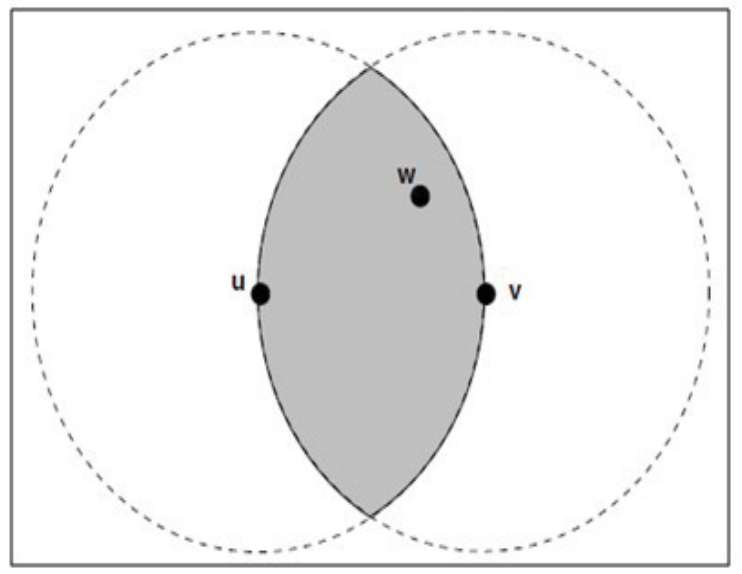

Figure 2: The edge $(u, v)$ not in $E$ because of $w$

algorithms use sub-graphs of $G$ to determine a topology to perform the broadcast operation. The frequently used sub-graphs are Relative neighborhood Graph (RNG) [6] and Local Minimum Spanning Tree (LMST) [6].

Given a wireless network represented by a graph $G=(V, E)$ where $V$ is the set of nodes and $E$ is set of links such that $E=\{(u, v) \mid u, v \in V$ and $d(u, v) \leq R\}$ where $R$ is the maximum transmission power of a node, the relative neighborhood graph of $G$ is denoted by $R N G(G)=\left(V, E_{r n g}\right)$ where, $E_{r n g}=\{(u, v) \mid ! \exists w \in V(u, w) \in E \wedge(v, w) \in E \wedge d(u, w)<$ $d(u, v) \wedge d(v, w)<d(u, v)\}$. As illustrated in Figure 2, an edge $(u, v)$ belongs to the RNG if there does not exist a node $w$ in the gray area. The gray area is the intersection of two circles centered at $u$ and $v$ with radii $d(u, v)$.

The Local Minimum Spanning Tree (LMST) is also a sub-graph of $G$. To determine LMST, each node $u$ computes of an MST in its own neighborhood denoted by $\operatorname{MST}(N(u))$. An edge between two nodes $u$ and $v$ exists in the LMST if and only if $u$ is a neighbor of $v$ in $M S T(N(v))$ and $v$ is a neighbor of $u$ in $M S T(N(u))$. LMST of a given graph $G=(V, E)$ is denoted by $\operatorname{LMST}(G)=\left(V, E_{l m s t}\right)$. For a given graph $G, \operatorname{LMST}(G)$ is a sub-graph of $R N G(G)$.

The authors of [7], proposed RNG Broadcast Oriented Protocol (RBOP). It uses the RNG to reduce the transmission power of nodes as much as possible and then applies the 
neighbor elimination technique to further reduce the redundant retransmissions. Experimental results show that it achieves performance comparable to the best known globalized BIP algorithm.

Cartigny et. el [6] proposed an extension of RBOP known as RBOP-T (RNG Broadcast Oriented Protocol with Full Timeout). In RBOP, only nodes receiving a packet on nonRNG edge apply timeout before retransmissions. In the case of RBOP-T, all nodes wait for a fixed amount of time before retransmitting a message. They also proposed another protocol named LBOP-T (Local MST Broadcast Oriented Protocol with Full Timeout). This protocol first replaces RNG in RBOP with Local MST and then applies a timeout before any node retransmits a message.

Li et al. [27] proposed a protocol named Broadcast on Local Minimum Spanning Tree (BLMST). In this technique an LMST is constructed and a broadcast message is relayed through the tree in constrained flooding fashion. Here if a node $v$ receives a message from all its neighbors in the LMST or knows that every neighbor has already received the message, it will not relay the message. The authors argued that as the LMST provides a minimally connected topology, applying further optimization rules to suppress the relay nodes will lead to marginal improvement. BLMST has several desirable features. BLMST is independent of the power consumption model. Since the LMST preserves network connectivity, the coverage under BLMST is $100 \%$. The control message overhead to get neighborhood information is not significant. Moreover, BLMST is scalable with increasing values of $n$.

Ingelrest et al. [20] considered that the minimal transmission energy required by a node $u$ so that the transmission can be received successfully by a neighbor $v$ at distance $r$ is proportional to $r^{\alpha}+c_{e}$, where $\alpha$ is a path loss component and $c_{e}$ is a factor considering the energy expenditure due signal processing, message reception, etc. They argued for the existence of optimal radius computed with hexagonal tiling of network area, that minimizes the energy consumption for a broadcast operation. The authors modified the existing LBOP [6] to take advantage of the optimal radius. This new protocol is named as Target Radius LMST Broadcast Oriented Protocol (TR-LBOP). As the node density increases, LBOP reduces transmission radii as LMST neighbors are getting closer. Short radii cause 
more nodes to act as relays. The constant energy charge $c_{e}$ for each transmission leads to huge energy consumption. LBOP is modified so that each node increases its transmission range up to the target optimal value when a retransmission is needed. Every node $u$ maintains two lists: $L(u)$ and $L^{\prime}(u) . L(u)$ contains LMST neighbors $v$ of $u$ and $L^{\prime}(u)$ stores every other neighbor of $u$. During the neighbor elimination scheme, each neighbor $v$ that receives the message is removed from either $L(u)$ or $L^{\prime}(u)$. When the timeout occurs, if $L(u)$ is empty, the retransmission is canceled. If there is at least one node in $L(u)$ node $u$ has to rebroadcast the message to reach the nodes left in $L(u)$. In this case, the authors defined two values $D_{L}$ and $D_{L^{\prime}}$ where $D_{L}$ is the length of the furthest LMST neighbor $v$ from $u$ and $D_{L^{\prime}}$ is the length of the edge between $u$ and its as yet unreached neighbor $w$ which is closest to optimal radius $T$. Finally the radius of $u$ is chosen to be the maximum of $D_{L}$ and $D_{L^{\prime}}$.

In Target Radius and Dominating Set Based Protocol (TRDS) [20], the radii of nodes are reduced to the target transmission radius $T$. This algorithm works in three steps.

1. The topology of the network is adapted in such a way that each node selects a transmission radius very close to $T$ and still maintains connectivity. For this purpose, a sub-graph of $G$ is constructed where each node considers only neighbors in RNG or LMST and the neighbors whose distance is less than or equal to $T$. The resulting sub graph $G_{T}$ is sparse, connected and bidirectional.

2. Given a connected graph $G_{T}$, a connected dominating set (CDS) is determined using any CDS algorithm. The size of the CDS is further reduced by computing the $R N G$ of the graph induced by the CDS. After this, every CDS node has just to cover its dominant neighbors in $R N G$.

3. For each node $u$, the set of CDS neighbors is denoted by $N_{D}(u)$ and the set of non-CDS neighbors is denoted by $N_{\bar{D}}(u)$. A CDS node $u$ wishing to launch a broadcast message emits its message with the minimal range that covers $N_{D}(u)$ and $N_{\bar{D}}(u)$. A non-CDS node $v$ that wishes to transmit a broadcast packet transmits its message to its nearest associated CDS neighbor $u$. A CDS node $u$ receiving a message rebroadcasts it with 
the range which allows to cover non-covered nodes in $N_{D}(u)$ and $N_{\bar{D}}(u)$. A non-CDS node $v$ will never relay messages.

The authors argued that several localized broadcasting protocols for minimizing energy consumption are proposed and they are based on selecting neighbors from a sparse topology. They do not consider the constant energy charge $c_{e}$ for each transmission. As a result, in the case of dense networks, these algorithms produce energy inefficient solutions. Both TRDS and TR-LBOP algorithms are efficient and give good results as compared to BIP for all network densities.

\subsection{Broadcasting in Duty Cycled WSN}

Broadcasting in wireless ad hoc networks has been intensively studied while broadcast in duty cycled wireless sensor networks is comparatively not as well-studied in the literature. We classified the broadcast algorithms for duty cycled WSNs into two categories: centralized and distributed. We first review the centralized algorithms in the following section and then discuss distributed algorithms in section 2.2.2.

\subsubsection{Centralized Algorithms}

Gu et al. [11] proposed the Dynamic Switch Forwarding (DSF) technique for low duty-cycle sensor networks with unreliable links in order to achieve optimal expected delivery ratio, expected end-to-end delay and expected energy consumption. In duty cycled networks, link quality-based forwarding techniques suffer from high end-to-end delay due to sleep latency. On the other hand, sleep latency based forwarding techniques suffer from high end-to-end delay due to the change of link quality. In DSF, given a sink, each node maintains a sequence of forwarding nodes that are sorted in the order of the wake-up time associated with them. To send a packet, a node scans the first node in the forwarding sequence as it will wake up soon and tries to send the packet. If the transmission is successful then the node stops. If it is unsuccessful, the node fetches the next node from the sequence and tries to send the packet again. The advantage of this technique is that it reduces the time spent on 

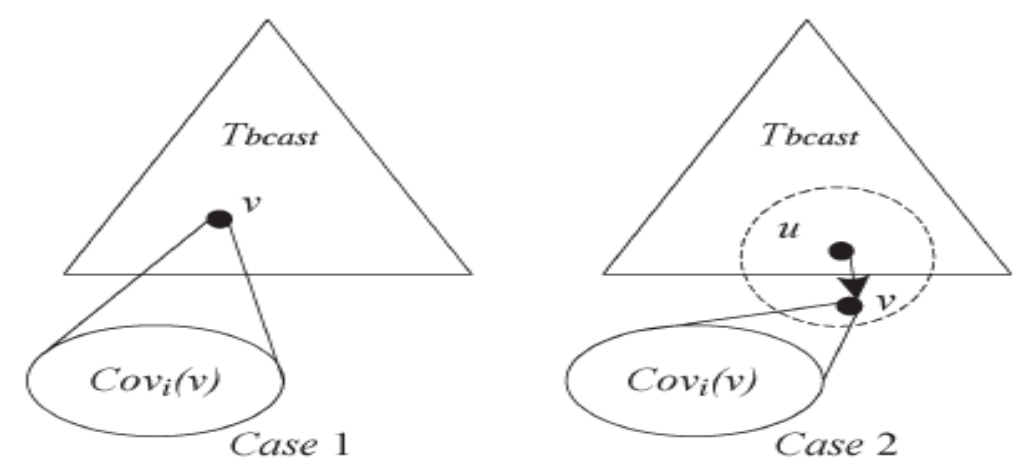

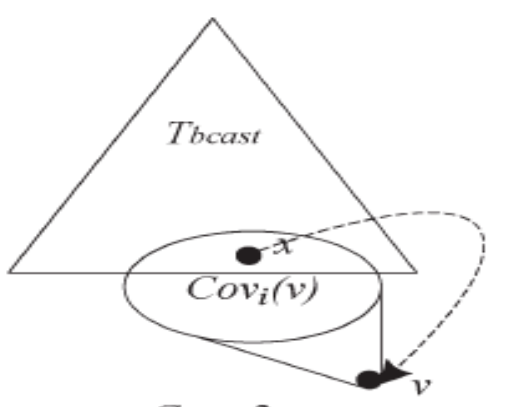

Case 3

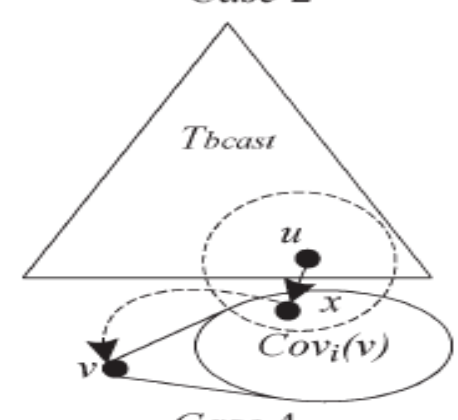

Case 4

Figure 3: Four cases of connecting a $\operatorname{Cov}_{i}(v)$ to the existing $T_{\text {bcast }}$ through $v$

transmitting a packet successfully by avoiding waiting for a particular forwarding node to wake up again after failure. The authors proposed three metrics: Expected Delivery Ratio (EDR), Expected End-to-End Delay (EED) and Expected Energy Consumption (EEC) and determined the model to calculate them for a given forwarding sequence. Later they provided dynamic programming algorithms to generate a forwarding subsequence that is optimal in terms of EDR, EED and EEC from the full forwarding sequence.

The authors of [18] considered the minimum transmission broadcast problem in uncoordinated duty-cycled wireless ad hoc or sensor networks and proved that this problem is NPComplete. They proposed a set-cover-based approximation scheme with both centralized and distributed approximation algorithms. ${ }^{1}$ The centralized set cover based approximation (CSCA) algorithm consists of two phases. Phase 1 determines a minimum covering node set. For each node $v, T(v)$ denotes the set of active time units in $\operatorname{Disk}(v)$, and $\operatorname{Cov}_{i}(v)$ denotes the set of nodes in $\operatorname{Disk}(v)$ with active time unit $i$. This algorithm groups all nodes

\footnotetext{
${ }^{1}$ Since in Chapter 4, we compare the performance of CSCA with our algorithms, we describe CSCA in some detail here.
} 
with active time slot $i$ in $G(V, E)$ into sets $U_{i}$ and tries to find a minimum covering node set $C_{i}$ for each $U_{i}$ in a greedy fashion so that $\cup_{v \in C_{i}} \operatorname{Cov}_{i}(v)=U_{i}$. Phase 2 then constructs a backbone structure by connecting all $\cup_{i \in T} C_{i}$ to $s$ through some connectors. The backbone is determined during the formation of a spanning tree $T_{b c a s t}$ on $G$. Initially, $T_{b c a s t}$ starts with $s$ and a working set Temp is set to $\cup_{i \in T} C_{i}$. This phase scans all the element in Temp and selects the first $\operatorname{Cov}_{i}(v)$ satisfying one of the following conditions as shown in Figure 3 and takes appropriate action:

1. $v$ is in $T_{b c a s t}$; In this case no operation is required.

2. $v$ is adjacent to some $u$ in $T_{b c a s t}$; In this case connect $u$ to $v$.

3. A node $x$ belongs to $\operatorname{Cov}_{i}(v) \cap T_{b c a s t}$; In this case connect $x$ to $v$.

4. A node $x$ in $\operatorname{Cov}_{i}(v)$ is adjacent to some $u \in T_{b c a s t}$; In this case connect $x$ to $u$ and $x$ to $v$.

Node $v$ is removed from Temp if all $\operatorname{Cov}_{i}(v)$ for $i \in T$ are processed. This process continues until Temp is empty.

The approximation ratio of the CSCA algorithm is shown to be $3(\ln (\Delta)+1)$, where $\Delta$ is the maximum degree of the network. The time complexity of the CSCA algorithm is $O\left(n^{3}\right)$.

In [46], the broadcast problem in a duty cycled wireless sensor network is considered as a shortest path problem in a time-coverage graph and an energy efficient centralized algorithm that utilizes dynamic programming is proposed. This algorithm saves energy by minimizing forwarding cost and delay. In this algorithm, at first a time-coverage graph is constructed. If a set $R$ of nodes receive a message at time $t$, it is represented as a vertex $v_{R, t}$ in the time-coverage graph. $R$ starts with $\{s\}$ and gradually becomes $\{1,2,3, \ldots, n\}$. There are two kinds of edges: time edges and forward edges. If nodes of $R$ do not forward messages at $t$, then same coverage state will exist in next time slot. This situation is depicted by a time edge that connects neighboring vertices along a row from earlier to later. A forwarding edge represents a forwarding event. A forwarding edge from $v_{R, t}$ to $v_{R^{\prime}, t^{\prime}}$ indicates that at 
time $t$, one or more nodes in $R$ transmit a message. The weight of an edge is a combination of message and time cost. When the weight of a time edge is calculated it emphasizes on the delay and the number of messages forwarded by the nodes in $R$ is highlighted during the calculation of the weights of forward edges. Let $W\left(v_{R, t}, v_{R^{\prime}, t^{\prime}}\right)$ denote the weight of an edge from $v_{R, t}$ to $v_{R^{\prime}, t^{\prime}}$ and $W\left(v_{R, t}, v_{R^{\prime}, t^{\prime}}\right)=\infty$ if no such edge exists. $F\left(v_{R^{\prime}, t^{\prime}}\right)$ is the total weight of the shortest path from $v_{s, t_{0}}$ to $v_{R^{\prime}, t^{\prime}} . F\left(v_{R^{\prime}, t^{\prime}}\right)$ is calculated as follows:

$$
F\left(v_{R^{\prime}, t^{\prime}}\right)=\min _{v_{R, t}}\left(F\left(v_{R, t}\right)+W\left(v_{R, t}, v_{R^{\prime}, t^{\prime}}\right)\right)
$$

where $F\left(v_{s, t_{0}}\right)=0$ and $F\left(v_{R, t_{0}}\right)=\infty$ for $R \neq s$. With the above relation and the boundary values, the weight of the shortest path from $v_{s, t_{0}}$ to each vertex from top to bottom and for each row, from left to right can be calculated. The minimum of the total weights to the last-row vertices is the weight of the shortest path from $v_{s, t_{0}}$ to a vertex in last row.

The problem of finding energy-efficient sleep scheduling that will optimize the end-to-end delay is addressed in [31]. The authors made an attempt to minimize the communication latency when each sensor has a duty cycling requirement of being awake for only $1 / k$ time slots on an average. As a first step, they considered each sensor can be active in exactly one time unit among the $k$ slots. They proved that finding a sleep scheduling that will minimize the end to end communication delay in a network with all-to-all communication flow and weighted communication flow is generally NP-hard. They found that an optimal solution can be obtained for two special cases of all-to-all communications: tree topologies and ring topologies. They proposed several heuristics for networks with all-to-all communication patterns. In the centralized algorithm, initially all nodes are assigned same slots. Each node calculates the delay diameter $D$ for all possible slot assignments for itself while keeping the time slots for other nodes fixed. The minimum of the delay diameters of all possible slot assignments is denoted by $d_{\min }$. If $d_{\min }$ is smaller than the previous delay diameter $d$, the node changes its slot to the one with minimum $D$ and updates $d$ to $d_{\text {min }}$. After all nodes perform the operation, the iteration can be repeated. The number of iterations depends on the time limitation of the algorithm.

The authors of [17] investigated the problem to find a broadcast schedule that avoids 
collisions and minimizes the broadcast latency in a duty cycled wireless ad hoc network and they proved that this problem is NP-Complete. They proposed two approximation algorithms named Simple Layered Coloring Algorithm (SLAC) and Enhanced Layered Coloring Algorithm (ELAC). Both of the two algorithms construct a broadcast tree using Dijkstra's algorithm for shortest paths where the cost of an edge $\lambda(u, v)$ is defined as follows:

$$
\lambda(u, v)=\left\{\begin{aligned}
|T| & \text { if } S L_{a}(v)=S L_{a}(u) \\
\left(S L_{a}(v)-S L_{a}(u)+|T|\right) \bmod |T|, & \text { otherwise }
\end{aligned}\right.
$$

where, $S L_{a}(u)$ and $S L_{a}(v)$ represent the active time slot of node $u$ and $v$ respectively and $T$ is the length of the wake up schedule.

If a node $u$ in the constructed tree has a receiving time slot $S L_{r}(u)=k|T|+i$, it is considered as a node at layer $k|T|+i$. The maximum $k$ is denoted by $K$ and the maximum $i$ is denoted by $I$ and the maximum layer is denoted by $K|T|+I$. Collisions will occur only when two or more nodes transmit to nodes in the same layer $p$ on the tree at the same time. SLAC schedules the the parents of nodes in layer $p$ to transmit in different time. For this purpose this algorithm utilizes the D2-coloring scheme. In D2-coloring scheme, no two vertices having distance 1 or 2 will not be assigned the same color [39]. The approximation ratio of SLAC is $O\left(\Delta^{2}+1\right)$, where $\Delta$ is the maximum degree of the network.

ELAC improves the SLAC algorithm by dividing the transmissions on each layer into two phases. Let $U_{p}$ denotes the set of nodes on layer $p$. ELAC constructs the Maximal Independent Set (MIS) $A_{p}$ for each $U_{p}$. This algorithm finds a proper $D$ - coloring of $A_{p}$. It finds the transmissions from the parents of $A_{p}$ to the nodes in $A_{p}$ and schedules transmissions from $A_{p}$ to the nodes in $U_{p}$. The approximation ratio of ELAC is $24|T|+1$ where $|T|$ is the number of time slots in a scheduling period.

The One-to-All-Broadcast (OTAB) algorithm is proposed in [22]. This algorithm reduces the broadcast latency and provides a collision free broadcast schedule. As in SLAC and ELAC, this algorithm also builds a shortest path tree with root at $s$ using Dijkstra's algorithm. OTAB assumes that $s$ starts the broadcast operation at time slot 0 . For every edge $(u, v)$, the latency $\operatorname{Lat}(u, v)$ is defined as follows: 


$$
\operatorname{Lat}(u, v)=\left\{\begin{aligned}
A(v)+1, & \text { if } u=s ; \\
A(v)-A(u), & \text { if } u \neq s \text { and } A(v)-A(u)>0 \\
A(v)-A(u)+|T|, & \text { otherwise }
\end{aligned}\right.
$$

OTAB constructs a broadcast tree $T_{B}$ based on the shortest path tree and schedules the broadcast accordingly. All the nodes in $V$ are grouped into different layers $L_{0}, L_{1}, \ldots, L_{D}$ according to their latency of the shortest paths from $s$, where $D$ is the maximum latency of all the shortest paths. Nodes at the same layer $L_{i}$ have the same active time slot. All the nodes except $s$ are divided into different sets $U_{0}, U_{1}, U_{2}, \ldots, U_{|T|-1}$ according to their active time slots. For each $U_{j}, 1 \leq j \leq|T|-1$, OTAB determines an MIS $Q_{j}$. All the nodes at $L_{i}, 0 \leq i \leq|T|-1$ are divided into two sets: an Independent Set(IS) $M_{i}$ and $L_{i} \backslash M_{i}$, where $M_{i} \in Q_{j}$ and $j$ equals $(i-1) \bmod |T|$. Each $U_{j}$ is divided into two sets $Q_{j}$ and $U_{j} \backslash Q_{j}$. Now $T_{B}$ is constructed using a layered approach. At each $L_{i}$, the parent nodes of $M_{i}$ are chosen from some nodes at higher layers. Some nodes of $M_{i}$ are selected as the parents of nodes in $L_{i} \backslash M_{i}$ and nodes at lower layers with the same active time slot. Then for each $U_{j}, 1 \leq j \leq|T|-1$, parents of $Q_{j}$ and $U_{j} \backslash Q_{j}$ are colored using $D 2$ - coloring methods. Transmissions from the parent nodes to their children nodes are scheduled based on the colors of the parent nodes. This scheduling starts at time slot 0 . At each layer $L_{i}$, the transmissions from the parent nodes for each node $u \in M_{i}$ are scheduled and finally the transmissions from the parent node of each $w$ in $L_{i} \backslash M_{i}$ are scheduled based on the coloring of the nodes. The approximation ratio of OTAB is at most $17|T|$ where $|T|$ is the number of time slots in a scheduling period.

We compare the results of our algorithms with the existing algorithms CSCA [18] and an adapted version of OTAB [22] named SDT(Shortest_Delay_Tree). We eliminate the phase of resolving collisions from OTAB for the purpose of fair comparison with our proposed algorithms. 


\subsubsection{Distributed Algorithms}

Gu et al. proposed the distributed version of DSF in [11]. The centralized version of the algorithm is discussed in Section 2.2.1. Initially, the sink node knows the values of EDR, EED and EEC and it is 1 for EDR, 0 for EED and EEC. The distributed DSF algorithm starts working by allowing the sink node to broadcast its EDR, EED and EEC values. When a node receives these information it starts to calculate its own EDR, EED and EEC utilizing the received information and determines the forwarding set for itself that optimizes a particular metric using the proposed dynamic algorithms. If the change in new values of EDR, EED and EEC exceeds a certain threshold compared to the old values of EDR, EED and EEC, the node will broadcast the new values. This process continues at a node until it does not receive any information about the updated expected values from all its neighbors.

The authors of [12] proposed a distributed flooding scheme for low-duty cycle wireless sensor networks with unreliable communication links. They constructed an energy optimal tree to reduce transmission redundancies and dissemination delay. The energy optimal tree is constructed by allowing a smaller hop count node to transmit to a larger hop count

node, where every node selects only one incoming link that has the best link quality among all its incoming links. These best quality links reduce the number of collisions as well as retransmissions of messages. The authors proposed a recursive equation to compute the distribution of forwarding delay for each node in a distributed way. From this a node $u$ determines its p-quantile delay $D_{p}$ as its threshold value and shares it with all the neighbors. If a node $v$ has a packet to forward to $u$, it calculates Expected Packet Delay (EPD) and compares with $D_{p}$. If $E P D<D_{p}$, the packet is delivered to $u$. In this way, a node $u$ in the tree can receive a packet from $v$ that is not its parent in the tree as $v$ can deliver the packet faster than $u$ 's parent. As the forwarding decision is distributed, it may happen that two or more nodes decide to transmit to a single node and the sending nodes cannot hear each other. To solve this problem a sender set for a given node is constructed where all nodes can hear each other to avoid collision and a back off technique is proposed to avoid collisions among the nodes in the sender set. Opportunistic flooding achieves significantly 
shorter flooding delay and consumes less transmission energy as compared to an improved version of traditional flooding implemented in duty cycled wireless sensor networks.

An adaptive algorithm to dynamically schedule message forwarding is proposed in [45]. It utilizes only local topology information and does not depend on global synchronization. It exploits overhearing to reduce message costs. This algorithm obtains near-optimal performance in terms of time and message costs and achieves high reliability.

Hong et al. [18] proposed the distributed version of CSCA algorithm described in 2.2.1. In Distributed Set Covered Approximation (DSCA) algorithm every node is initially in idle listening state and turns to duty-cycling mode when a trigger variable $z$ is set from 0 to 1 . An $i$-dominator is a covering node for $U_{i}$ and an $i$-dominatee is a node in $U_{i}$ covered by some $i$-dominator. The authors used the term $*$-dominator and $*$-dominatee to represent any $i$-dominator and $i$-dominatee. In order to distributively implement the algorithm, the $i$-dominator is elected from $U_{i}$. Each node has its 2-hop neighbor information. Initially, all nodes are marked in white and then turned to blue if they become $i$-dominators or $i$ dominatees. Every white node with active time slot $i$ broadcasts the IamDominator $(I D, i)$ message and is marked blue if it has the most white neighbor nodes with active time slot $i$ among all its 1-hop white neighbors. A white node with active time slot $i$ also becomes an $i$-dominator and is marked blue if it has neither white neighbors with active time slot $i$ nor $i$-dominators. A white node with active time slot $i$ becomes an $i$-dominatee and is marked blue when it receives IamDominator $(I D, i)$ message and broadcasts the IamDominatee $(I D, i)$ message. At the end, each node is a $*$-dominator or a $*$-dominatee and all nodes in $U_{i}$ are covered by $i$-dominators.

Next phase determines the connections among $i$-Dominators and $s$. A forwarding tree $H$ connecting all $i$-dominators and $s$ is constructed by exchanging the $I N V / J O I N$ messages for all $i \in T$. Each node maintains a local trigger variable $z$ that is set from 0 to 1 after this node joins $H$. The approximation ratio of the DSCA algorithm is a constant of at most 20. Both the time and the message complexities of the DSCA algorithm are $O(n)$.

The Asynchronous Duty-Cycle Broadcasting (ADB) protocol for wireless sensor networks using asynchronous duty cycling is proposed in [43]. This protocol is integrated with 
MAC layer to utilize the information available at this layer. It optimizes broadcast operation at level of individual transmission of a node to a neighbor. ADB utilizes unicast transmission to every neighbor and waits for an acknowledgment from the receiving node. It uses a receiver- initiated mechanism to prevent a node from occupying the medium for too long a time. Whenever a neighbor wakes up, it sends a beacon message. After receiving the beacon message the node can send the data packet. While a node is waiting for the beacon before transmitting data, the medium can be utilized by the neighbors of the node that already received the packet to transmit to their neighbors in order to reduce delivery latency. ADB efficiently incorporates the progress of broadcast operation with the data packet to reduce number of transmissions by a node. It makes use of the best quality link to reduce energy consumption, delivery latency and to increase delivery ratio.

Thus ADB has certain properties making it energy efficient, reduce delivery latency and increase delivery ratio. They are:

1. ADB allows a node to go to sleep as soon as all its neighbors are reached or delegated to other nodes.

2. It avoids transmissions on poor links.

3. It prevents a node to occupy the medium for long time.

4. When a node wakes up, it is informed about the progress of broadcast with the data packet to avoid unnecessary waiting and transmissions.

The authors confirmed through experiments that ADB is highly energy efficient, reduces network load and delivery latency while achieving $99 \%$ delivery ratio.

Wang et al. [46] proposed the distributed version of finding shortest path in timecoverage graph. The centralized version of the algorithm is discussed in 2.2.1. From the solution of the centralized algorithm the authors derived an efficient and scalable distributed algorithm that utilizes local information and is associated with loss compensation techniques. For each sensor node this algorithm will determine the optimal forwarding sequence covering its 2-hop neighbors. Each node $w$ determines CovSet consisting of its 
1-hop and 2-hop neighbors known by $w$ being covered by at least one forwarding. When $w$ forwards a message or overhears a neighbor is doing so will update the CovSet. The centralized dynamic programming algorithm is modified so that every node $w$ starts from the row with index equal to its CovSet and the index of the last row will contain only the node $w$ and its 1- and 2-hop neighbors.

From simulations it is observed that the distributed algorithm achieves a near optimal performance and its time cost as well as the message cost is very close to the lower bound of the time cost and the message cost. Its computation and control overheads both scale well with the network size and density. It is also robust against wireless losses and cope well with different duty-cycles.

Stann et al. [40] proposed the Robust Broadcast Protocol (RBP) to improve the reliability of the broadcast operation while maintaining energy efficiency. This protocol requires only local information. It makes a single broadcast more reliable and hence decreases the frequency with which an upper layer protocol needs to invoke flooding. Thus better reliability of the broadcast operation improves the energy consumption. In RBP, every node knows its 1-hop neighbors. RBP generates a unique identifier when a new broadcast message is initiated. It must understand when broadcasts by different nodes correspond to the same flood. When a node hears a broadcast for the first time, it retransmits the packet unconditionally. When other neighbors also transmit the same packet, the node keeps track of which neighbors have broadcasted the packet. RBP considers the transmission by a neighbor as an implicit ACK. When the number of implicit ACKs observed by a node falls below a predetermined threshold, a node will again retransmit the packet. A receiver also sends an explicit unicast ACK when it hears a repeated broadcast from the same sender. RBP can switch a node from broadcast mode to unicast in order to reduce the number of packet transmissions. RBP adjusts both the retransmission thresholds and the number of retries based on neighborhood density. Higher density in the neighborhood implies lower thresholds and fewer retries as other neighbors are likely to broadcast the same packet. RBP can identify important links that bridge between dense clusters of nodes with sparse area of nodes. Often there can be a node $v$ at the edge of the dense area with large number 
of neighbors but $v$ alone provides traffic to the sparse area. RBP identifies such links and always ensures reliability for these links.

Experimental results show $99.8 \%$ reliability is achieved with overhead less than $48 \%$ as compared to the level of flooding required to get the same reliability.

Lu et al. [31] proposed two localized algorithms to find a sleep schedule that will minimize end-to-end delay in networks with all-to-all communications. In the first algorithm, a node only knows the slot assignments of its immediate neighbors and selects one slot for itself that will minimize the maximum delay to and from its 1-hop neighbors. This process is repeated several times. The second algorithm works in similar fashion as the Distance Vector routing technique. Each node maintains a forward vector table FDV which stores its shortest delays to all other nodes and a backward table BDV which stores its shortest delays from all other nodes. These two tables can be calculated using the basic Bellman-Ford technique. A sensor node knows the DV tables of its immediate neighbors and calculates the DV tables for all possible new slot assignments for itself. The maximum value of entries in the sets of the two DV tables over all possible slot assignments is denoted by maxd. The node selects the slot which gives the minimum maxd. In the randomized algorithm, a slot for each node is selected randomly and the delay diameter of the network is determined. After a fixed number of iterations, every node selects a time slot that gives the minimum delay diameter. The proposed heuristics are evaluated through simulations. It is found that the performance of the localized heuristics is worse than the above simple randomized slot allocations, while the centralized scheme provides more delay reductions over randomized schemes.

\subsubsection{Differences with Our Work}

Due to energy constrained nature of WSNs, broadcast algorithms for the duty cycled wireless sensor networks mainly focus on reducing the energy consumption. Various algorithms put emphasis on various aspects in order to achieve this goal. The Algorithm in [18] minimizes the number of node transmissions in uncoordinated duty cycled WSNs, while the algorithm in [46] converts the broadcast problem into a shortest path problem in timecoverage graph and saves energy by reducing forwarding cost and delay. The authors of [12] 
reduces transmission redundancies and dissemination delay by building an energy optimal tree where every node selects the best quality link with its parent in the tree. They also provide a technique to avoid collisions. The ADB algorithm in [43] achieves energy efficiency by allowing a node to go back to sleep as soon as all it neighbors are reached or delegated to other node. Here a node remains active after receiving a packet until it goes back to sleep. SLAC [17], ELAC [17] and OTAB [22] provide a collision free broadcast schedule that will minimize the broadcast latency.

None of the above algorithms for duty cycled wireless sensor networks consider the issue of minimizing the total number of additional active time units that nodes of a network need to be active in order to accomplish the broadcast operation. In our work we prove that the problem of reducing the total additional active time units for broadcast operation is NP-Complete and we propose two polynomial time heuristic algorithms to address this problem. 


\section{Chapter 3}

\section{Algorithms and NP-Completeness}

Energy efficient broadcast operation in duty cycled WSNs can be achieved by reducing the total number of additional active time units that the nodes of a network must be awake to complete the broadcast operation. We call the problem of minimizing the total number of additional active time units to accomplish a broadcast operation in a duty cycled WSN as the Minimum Energy Broadcast Tree (MEBT) problem. We prove that the MEBT problem is NP-Complete and propose two polynomial time heuristic algorithms to find suitable solutions for it.

In this chapter we first present the NP-Completeness proof of MEBT problem that is followed by the heuristic algorithms to address the MEBT problem. All of the algorithms construct a spanning tree rooted at a source node to accomplish the broadcast operation by minimizing the total additional active time units.

\subsection{Definitions and Preliminaries}

A Duty Cycled WSN is a triple $G=(V, E, M)$ where $V$ is the set of nodes in the network, $E \subseteq V \times V$ is the set of links and $M_{u}$ is the Wakeup Schedule of node $u$ and is a binary 
array of length $n$ where,

$$
M_{u}[i]= \begin{cases}1 & \text { if node } u \text { is in active state in time unit } i \\ 0 & \text { otherwise }\end{cases}
$$

Our goal is to provide broadcast service with minimum energy consumption in duty cycled WSNs. To accomplish this task usually a broadcast tree is constructed. A broadcast tree is a directed spanning tree rooted at a source node such that a packet from the source node can be sent to every other node in the network using the edges of the tree. Since a parent node has to stay awake until it delivers the packet to all its children in the tree, the number of active time units for the parent node is usually greater than that specified in its wakeup schedule.

In other words, the broadcast tree dictates a new schedule for each node, which we call the Broadcast Schedule. The broadcast schedule of a node is a binary array $B$ of length $n d$ where, $d$ is the depth of the tree. Let the extended wakeup schedule of a node be specified by an array $M_{u}^{\prime}$ of size $n d$, which consists of $d$ consecutive copies of the wakeup schedule; this specifies when the nodes would be awake in a time period of nd according to their wakeup schedules. Suppose a node $u$ receives the broadcast packet at time $t_{0}$ and it has $k$ children in the broadcast tree. Let $t_{i}$ be the next time unit when a child $v_{i}$ is awake, then the number of time units $u$ will remain active is $\max \left\{t_{i}-t_{0} \mid 1 \leq i \leq k\right\}$. The broadcast schedule for $u$ is defined as follows:

$$
B_{T}(u, t)=\left\{\begin{aligned}
1 & \text { if } t_{0} \leq t \leq \max _{1 \leq i \leq k} t_{i} \\
M_{u}^{\prime}(t) & \text { otherwise }
\end{aligned}\right.
$$

It is easy to see that a parent delivers a packet to all its children within $n$ time units after receiving it. Clearly for any node $u, \sum_{i=0}^{n d} B_{T}(u, i) \geq \sum_{i=0}^{n d} M_{u}^{\prime}[i]$. We define the cost of a broadcast tree $T$ to be $\operatorname{cost}(T)=\sum_{u=1}^{N} \sum_{i=0}^{n d}\left(B_{T}(u, i)-M_{u}^{\prime}[i]\right)$. In other words, the cost of a broadcast tree is the total number of additional time units that the nodes in the tree remain awake in order to accomplish the broadcast. The problem that we are interested in is the Minimum Energy Broadcast Tree (MEBT) problem, that is: Given a duty cycled 
network $G=(V, E, M)$ and a source node $s \in V$, find a broadcast tree $T$ rooted at $s$ for $G$ such that $\operatorname{cost}(T) \leq \operatorname{cost}\left(T^{\prime}\right)$ for all broadcast trees $T^{\prime}$ for $G$.

In our experiments, we also consider additional cost measures that have been studied previously such as the minimum number of node transmissions, the maximum delay and average delay dictated by a broadcast tree.

\subsection{NP-Completeness of MEBT Problem}

In this section, we show that the decision version of the MEBT problem is NP-Complete.

Theorem 1. Given a duty cycled wireless sensor network $G=(V, E, M)$ and a source node $s \in V$, the problem of determining if there exists a broadcast tree for $G$ rooted at $s$ with cost at most $W$ is NP-complete.

Proof. Given a candidate broadcast tree $T$ rooted at a source node, we can calculate the cost and check whether it is greater than $W$ in polynomial time. So the problem is in NP. We consider a special case of the problem, where the wakeup schedule of each node consists of 2 time units. We will show that the problem is NP-complete for this special case by reducing the 1-in-3 SAT problem to it and thus also NP-complete for more general case.

Let the 1-in-3 SAT instance consist of $n$ boolean variables $x_{1}, x_{2}, \ldots, x_{n}$ and $m$ clauses $C_{1}, C_{2}, \ldots, C_{m}$ where $C_{j}=y_{j, 1} \bigvee y_{j, 2} \bigvee y_{j, 3}$ and the three literals $y_{j, 1}, y_{j, 2}, y_{j, 3} \in\left\{x_{1}, \bar{x}_{1}, x_{2}, \bar{x}_{2}\right.$ $\left.\ldots, x_{n}, \bar{x}_{n}\right\}, 1 \leq j \leq m$. If the instance is satisfiable, then in each clause $C_{j}$, exactly one of $y_{j, l}$ is true, while the other two literals are false, $1 \leq j \leq m, 1 \leq l \leq 3$. We construct the MEBT instance $G=(V, E, M)$ corresponding to this 1-in-3 SAT instance. The vertices and their wakeup schedules are specified as follows:

- There is a source node $S_{0,0}$ where $M_{S_{0,0}}[1]=1$ and $M_{S_{0,0}}[2]=0$.

- For each boolean variable $x_{i}$ there are two corresponding nodes $S_{i, 1}$ and $S_{i, 2}$ and $M_{S_{i, j}}[1]=1$ and $M_{S_{i, j}}[2]=0$, where, $1 \leq i \leq n, 1 \leq j \leq 2$.

- For each boolean variable $x_{i}$ there is an additional node $X_{i}$ with $M_{X_{i}}[1]=0$ and $M_{X_{i}}[2]=1$, where, $1 \leq i \leq n$. 


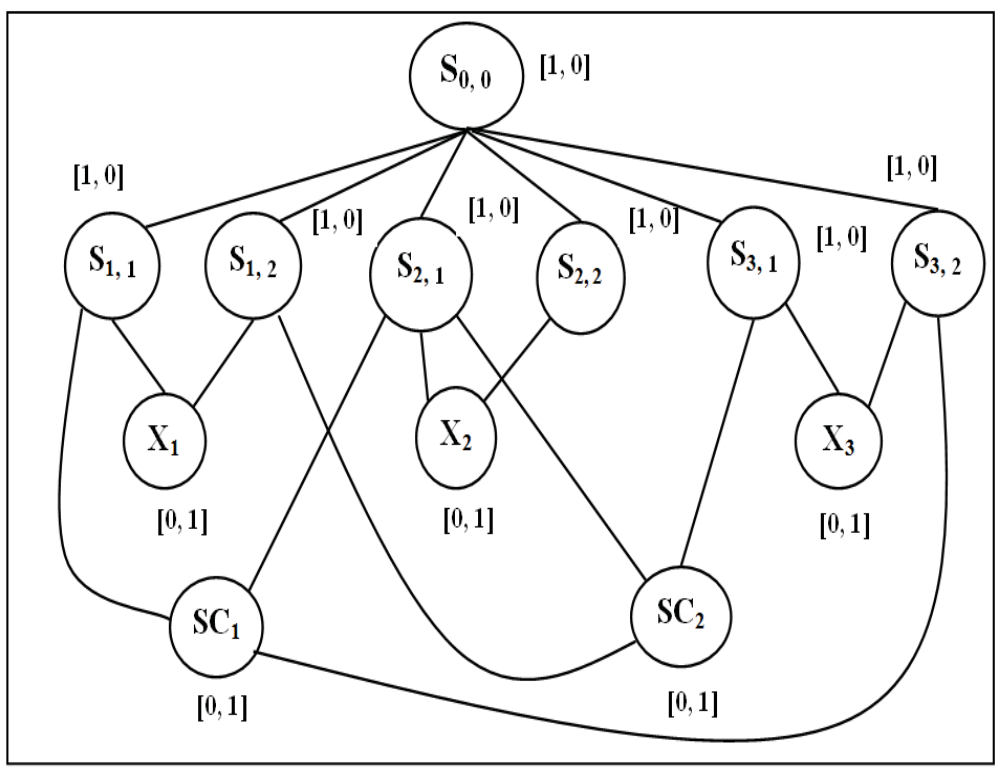

Figure 4: $G$ for variables $x_{1}, x_{2}, x_{3}$ and $C_{1}=x_{1} \bigvee x_{2} \bigvee \bar{x}_{3}, C_{2}=\bar{x}_{1} \bigvee x_{2} \bigvee x_{3}$

- For each clause $C_{j}$, there is a corresponding node $S C_{j}$ and $M_{S C_{j}}[1]=0$ and $M_{S C_{j}}[2]=$ 1 , where, $1 \leq j \leq m$.

Now the set of edges $E$ is constructed as follows:

- Every node $S_{i, j}$ is within the transmission range of $S_{0,0}$, where, $1 \leq i \leq n, 1 \leq j \leq 2$.

- $X_{i}$ is within the transmission range of $S_{i, 1}$ and $S_{i, 2}$, where, $1 \leq i \leq n$.

- $S C_{j}$ is within transmission range of $S_{i, 1}$ when $x_{i}$ is a literal of $S C_{j}$, where, $1 \leq i \leq$ $n, 1 \leq j \leq m$. It is within transmission range of $S_{i, 2}$ when $\bar{x}_{i}$ is a literal of $S C_{j}$, where, $1 \leq i \leq n, 1 \leq j \leq m$

A similar graph was used in [28] to address a different problem. Figure 4 shows $G$ for 1-in-3 SAT instance consisting of variables $x_{1}, x_{2}, x_{3}$ and $C_{1}=x_{1} \bigvee x_{2} \bigvee \bar{x}_{3}, C_{2}=\bar{x}_{1} \bigvee x_{2} \bigvee x_{3}$

We claim that the MEBT instance has a broadcast tree of cost $n$ if and only if the original 1-in-3 SAT instance has a satisfying assignment.

Now suppose we are given a satisfying assignment of 1-in-3-SAT. We will construct a directed broadcast tree $T$ rooted at $S_{0,0}$ such that the cost of $T$ is no greater than $n$. First we choose $S_{0,0}$ as root. All edges $\left\langle S_{0,0}, S_{i, j}>\right.$ are included in $T$ for all $i, j$ so 


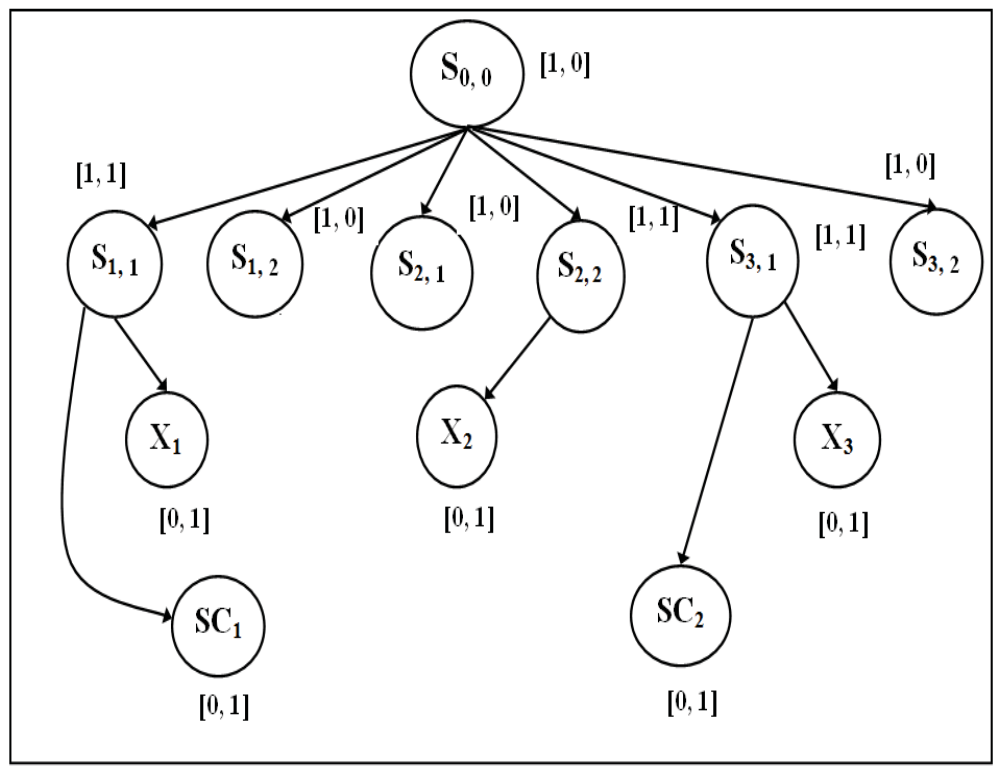

Figure 5: Broadcast Tree of $G$ with $W=3$

that $S_{i, j}$ is able to receive packets sent by $S_{0,0}$ in first time unit. Thus, $B_{T}\left(S_{i, j}, 1\right)$ is 1 , $1 \leq i \leq n, 1 \leq j \leq 2$ and $B_{T}\left(S_{0,0}, 1\right)=1$ and $B_{T}\left(S_{0,0}, 2\right)=0$. For each boolean variable $x_{i}$, if it is true in the assignment, then $\left\langle S_{i, 1}, X_{i}\right\rangle$ is in $T$ and $B_{T}\left(S_{i, 1}, 2\right)$ is 1 and, otherwise it is $0,1 \leq i \leq n$. On the other hand, if the value of $x_{i}$ in the assignment is false, then $<S_{i, 2}, X_{i}>$ is in $T$ and $B_{T}\left(S_{i, 2}, 2\right)$ is 1 , otherwise it is $0,1 \leq i \leq n . B_{T}\left(X_{i}, 1\right)$ is 0 and $B_{T}\left(X_{i}, 2\right)$ is 1 in order to receive a packet from either $S_{i, 1}$ or $S_{i, 2}, 1 \leq i \leq n$. Clearly, all $X_{i}$ are reached. For a given clause $C_{j}=y_{j, 1} \bigvee y_{j, 2} \bigvee y_{j, 3}$, exactly one literal $y_{j, l}$ is true, $1 \leq j \leq m, 1 \leq l \leq 3$. If $y_{j, l}=x_{i}$ is true in the assignment, then the directed edge $\left\langle S_{i, 1}, S C_{j}>\right.$ is included in $T, 1 \leq i \leq n, 1 \leq l \leq 3,1 \leq j \leq m$ and $B_{T}\left(S_{i, 1}, 2\right)$ and $B_{T}\left(S C_{j}, 2\right)$ are 1 . Otherwise if $y_{j, l}=\bar{x}_{i}$ is true in the assignment, then the directed edge $<S_{i, 2}, S C_{j}>$ is in $T, 1 \leq i \leq n, 1 \leq j \leq m, 1 \leq l \leq 3$ and $B_{T}\left(S_{i, 2}, 2\right)$ and $B_{T}\left(S C_{j}, 2\right)$ are 1 .

$T$ is a spanning tree rooted at $S_{0,0}$ as it is acyclic and connects all the nodes. $B_{T}\left(S_{0,0}, 1\right)$ and $B_{T}\left(S_{i, j}, 1\right)$ are 1 as $S_{i, j}$ will receive packets from $S_{0,0}$ at the first time unit, $1 \leq$ $i \leq n, 1 \leq j \leq 2 . \quad B_{T}\left(S_{i, j}, 2\right)$ of $n S_{i, j}$ is 1 in order to send the message to $X_{i}$ and $S C_{k}, 1 \leq i \leq n, 1 \leq j \leq 2,1 \leq k \leq m$. So, the cost of $T$ is $n$. Figure 5 shows the broadcast tree of cost 3 for $G$ in Figure 4 corresponding to the assignment $x_{1}=T, x_{2}=F$ and $x_{3}=T$.

Now suppose there is a broadcast tree $T$ rooted at $S_{0,0}$ with cost no greater than $n$. We 
want to show that we can derive a satisfying assignment for the 1-in-3 SAT instance. First observe that since $T$ is a directed spanning tree, and since every $X_{i}$ is connected only to $S_{i, 1}$ and $S_{i, 2}$ in the graph, exactly one of the links $\left(S_{i, 1}, X_{i}\right)$ and $\left(S_{i, 2}, X_{i}\right)$ must be present in the tree, where $1 \leq i \leq n$. Since both $S_{i, 1}$ and $S_{i, 2}$ are only awake in the first time unit, and $X_{i}$ only in the second, the presence of these links alone gives a cost of $n$ to the tree. Therefore, any other links in the tree must come for free; they must not add any cost to the tree. Therefore, the parent of any node $S C_{j}$ in the tree must be a node $S_{i, 1}$ or $S_{i, 2}$ that is also the parent of its corresponding $X_{i}$, where $1 \leq i \leq n, 1 \leq j \leq m$. That is, if $\left(S_{i, 1}, S C_{j}\right)$ is a link in the tree, then $\left(S_{i, 1}, X_{i}\right)$ is present as well.

To obtain a satisfying assignment now is easy. For each $i$, if $\left(S_{i, 1}, X_{i}\right)$ is in the tree, then the corresponding variable $x_{i}$ in the 1-in-3 SAT instance is assigned the value True, otherwise $\left(S_{i, 2}, X_{i}\right)$ is in the tree, and $x_{i}$ is assigned the value False, $1 \leq i \leq n$. Clearly this is a valid assignment. Finally, since $T$ is a directed spanning tree, every $S C_{j}$ has exactly one incoming link, $1 \leq j \leq m$. If the incoming link is from a node $S_{i, 1}$, then the literal $x_{i}$ is in the clause $C_{j}, 1 \leq i \leq n, 1 \leq j \leq m$. Recall that in this case, the edge it has been assigned the value True, the clause will be satisfied. On the other hand, if the incoming link is from a node $S_{i, 2}$, then the literal $\overline{x_{i}}$ is in the clause $C_{j}, 1 \leq i \leq n, 1 \leq j \leq m$. Since in this case, the variable $x_{i}$ has been assigned False, the clause will be satisfied. Thus we have a satisfying assignment with exactly one true literal per clause as required.

\subsection{Algorithms}

In this section we present our polynomial time algorithms to find a broadcast tree with low cost. We will use a simple duty cycled WSN $G$ to describe the operation of our proposed algorithms. The duty cycled WSN $G$ used for this purpose is shown in Figure 6. It consists of 11 nodes. The length of the wakeup schedule is 6 and every node is active for exactly one time unit in this schedule. The nodes are labeled starting from $a$. We select node $a$ as the source node responsible for initiating the broadcast operation. 


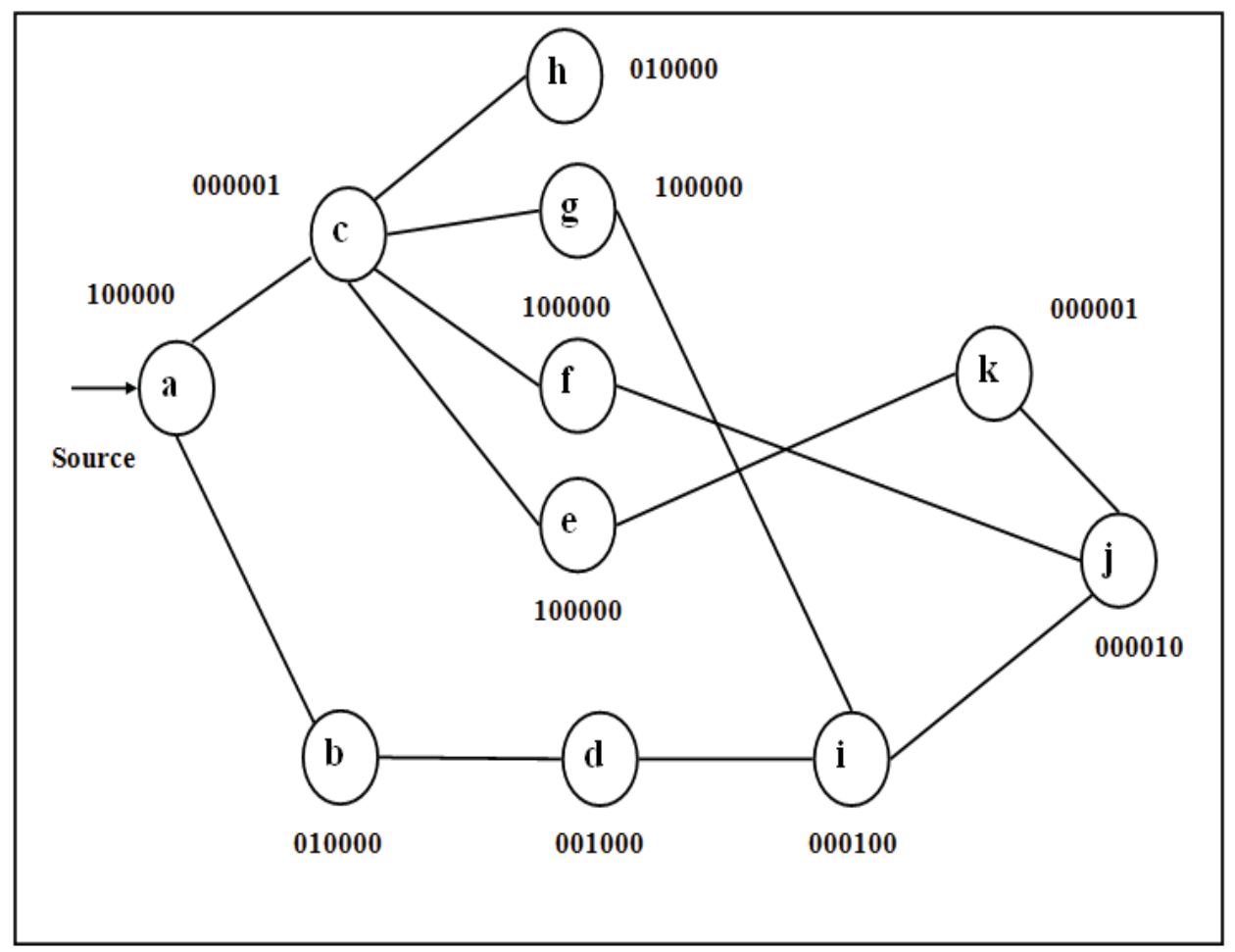

Figure 6: Example of a duty cycled WSN $G$ used to illustrate algorithms

\subsubsection{Spanning Tree with Incremental Cost (STIC) Algorithm}

We propose a new algorithm Spanning Tree with Incremental Cost (STIC) that is similar to Prim's algorithm for minimum cost spanning tree [35], and differs only in that the costs of edges are dynamically updated. It is important to note that our algorithm creates a spanning tree of the original graph, but not necessarily a minimum spanning tree. A similar idea was used in [49], but with a different notion of cost. As in Prim's algorithm, we iteratively add the lowest cost node to the tree, starting with the root node. The cost of an edge $(u, v)$ is initialized to $w(u, v)=t^{\prime}-t \quad(\bmod n)$, where $t$ and $t^{\prime}$ are the active time units of $u$ and $v$ respectively and the subtraction is done in modulo $n$ arithmetic. However, when a node is added to the tree, the number of additional time units that its parent $u$ needs to stay awake in order to transmit the message to its other children, say $v$ not yet in the tree is reduced. 


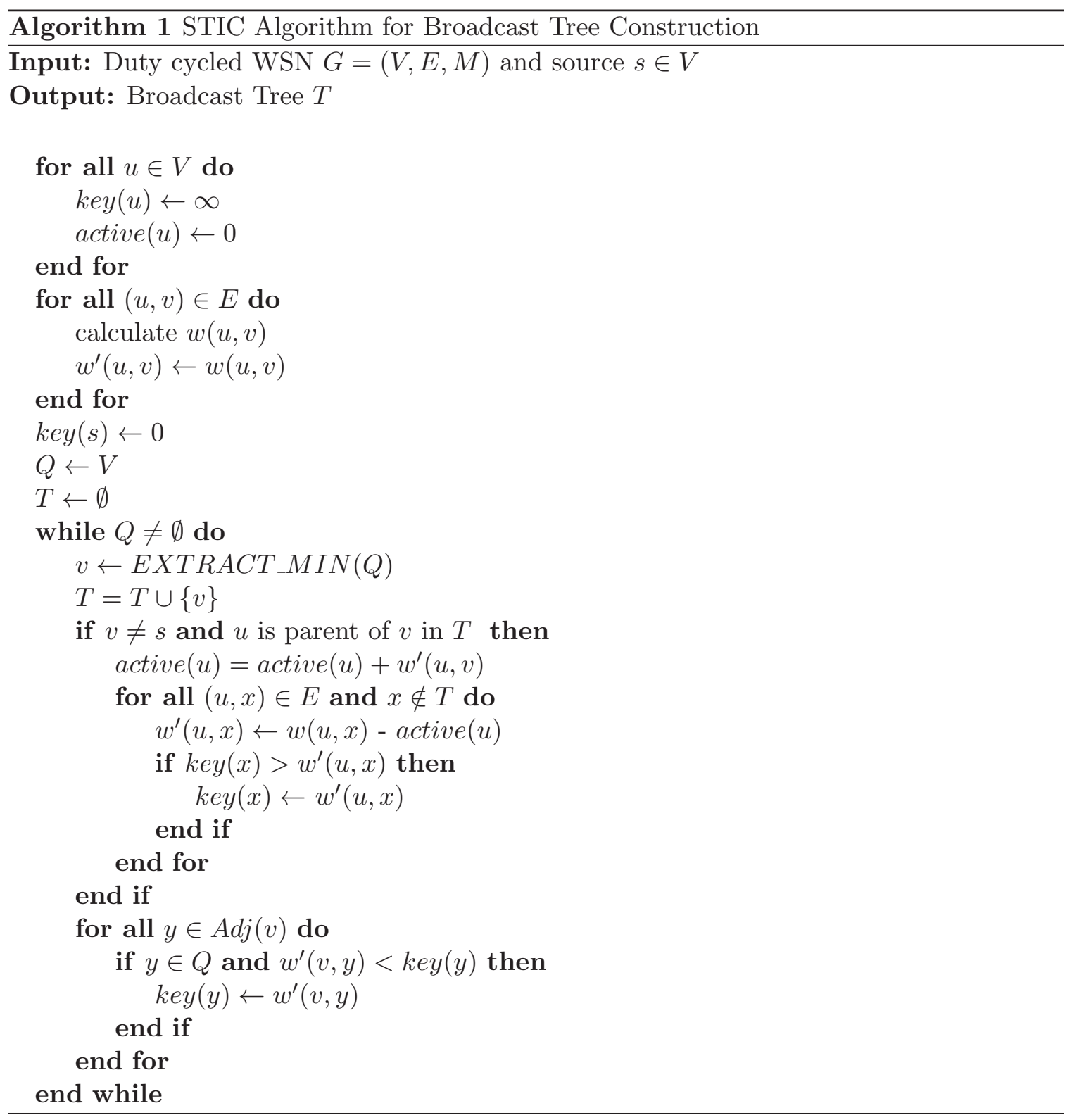

The cost of those edges is therefore updated to $w^{\prime}(u, v)=w(u, v)$-active $(u)$, where $\operatorname{active}(u)$ is the number of time slots for which $u$ was already active. In every iteration, an edge with lowest $w^{\prime}(u, v)$ is added into $T$. When an edge $(u, v)$ is added to the broadcast tree, the cost of $u$ 's edges to its other neighbors not in $T$ changes and is updated accordingly before proceeding to the next iteration. This process continues until all nodes are in $T$. A broadcast schedule is dictated by the tree and the cost is computed. The complexity of the algorithm is $O(m \lg N)$ when $G$ is sparse and it is $O\left(N^{2}\right)$ when $G$ is dense, where $N$ is the 
number of nodes and $m$ is the number of edges.

On our example network of Figure 6, STIC starts with node $a$ and constructs $T$ in a number of steps. The construction of $T$ is shown in Figures 7, 8, 9, 10, 11 and 12 . To conveniently illustrate the procedure to generate the tree, each node $u$ is labeled with $\operatorname{key}(u) / \operatorname{active}(u)$ inside the circle whereas each edge $(u, v)$ is labeled with $w(u, v) / w^{\prime}(u, v)$. A bold directed arrow from $u$ to $v$ indicates that $(u, v)$ is included into $T$. The construction process is described below:

1. In Figure $7(\mathrm{~b})$, initially $k e y(a)=0$ and thus node $a$ is added into $T$. Node $b$ and $c$ are adjacent to $a$ and $k e y(c)$ and $k e y(b)$ are updated to 5 and 1 , respectively as $w(a, b)=w^{\prime}(a, b)=1$ and $w(a, c)=w^{\prime}(a, c)=5$.

2. As shown in Figure $8(a)$, node $b$ is extracted as $(a, b)$ is the lowest cost edge with $w^{\prime}(a, b)=1$ and active $(a)$ becomes 1 as 1 is added to its previous value. Node $a$ is the parent of $c$ and thus $w^{\prime}(a, c)$ becomes 4 as $w^{\prime}(a, c)=w(a, c)-\operatorname{active}(a)=5-1=4$ and $\operatorname{key}(c)$ is updated to 4 . Node $d$ is adjacent to $b$ and thus $k e y(d)$ becomes 1 as $w^{\prime}(b, d)$ is 1 .

3. In Figure $8(\mathrm{~b})$, node $d$ is selected to be included into $T$ as $(b, d)$ is the lowest cost edge and active $(b)$ becomes 1 . As there are no more edges incident to $b$, there is no update of $w^{\prime}$. Node $i$ is adjacent to $d$ and $\operatorname{key}(i)$ becomes 1 as $w^{\prime}(d, i)$ is 1 .

4. As shown in Figure $9(\mathrm{a})$, edge $(d, i)$ is the lowest cost edge and thus $i$ is added into $T$ and active $(d)$ becomes 1 . Node $g$ and $j$ are adjacent to $i$ and $k e y(g)$ and $k e y(j)$ are updated to 3 and 1 , respectively as $w^{\prime}(i, g)=3$ and $w^{\prime}(i, j)=1$.

5. As shown in Figure 9(b), node $j$ is included into $T$ as $(i, j)$ is the lowest cost edge and active $(i)$ becomes 1 . Node $i$ is the parent of $g$ and thus $w^{\prime}(i, g)$ becomes 2 as $w^{\prime}(i, g)=w(i, g)-\operatorname{active}(i)=3-1=2$ and $k e y(g)$ becomes 2 . Node $k$ and $f$ are adjacent to $j$ and thus $k e y(k)$ and $k e y(f)$ become 1 and 2 , respectively.

6. In Figure 10(a), Node $k$ is added into $T$ as $k e y(k)=1$ and $\operatorname{active}(j)$ becomes 1 . As $j$ is parent of $f, w^{\prime}(j, f)$ as well as $k e y(f)$ become 1 . Node $e$ is adjacent to $k$ and $k e y(e)$ 
is updated to 1 as $w^{\prime}(k, e)$ is 1.

7. As shown in Figure 10(b), key(f) and key(e) are both 1. To break the tie, we arbitrarily select node $e$ to add into $T$. Thus edge $(k, e)$ is included into $T$ and active $(k)$ becomes 1 . Node $c$ is adjacent to $e$ but $k e y(c)<w^{\prime}(e, c)$ as $w^{\prime}(e, c)=5$ and $k e y(c)=4$ and hence $k e y(c)$ retains the current value.

8. In Figure 11(a), Now node $f$ is added into $T$ as $(j, f)$ is the lowest cost edge and active $(j)$ becomes 2 . As $k e y(c)<w^{\prime}(f, c)$, key $(c)$ retains the current value.

9. As depicted in Figure 11(b) node $g$ is added into $T$ as $(i, g)$ is the lowest cost edge and active $(i)$ becomes 3. As $k e y(c)<w^{\prime}(g, c)$, key $(c)$ does not change the current value.

10. As shown in Figure 12(a) node $c$ is included into $T$ as $(a, c)$ is the lowest cost edge and active $(a)$ becomes 5 . As $h$ is the only neighbour of $c$ not in $T$, key $(h)$ becomes 2 .

11. Finally in Figure 12(b) node $h$ is added into $T$ and active $(c)$ becomes 2.

The final cost of $T$ is obtained by adding up the active $(u)$ for all $u \in V$ and $\operatorname{cost}(T)=15$ for this example. 


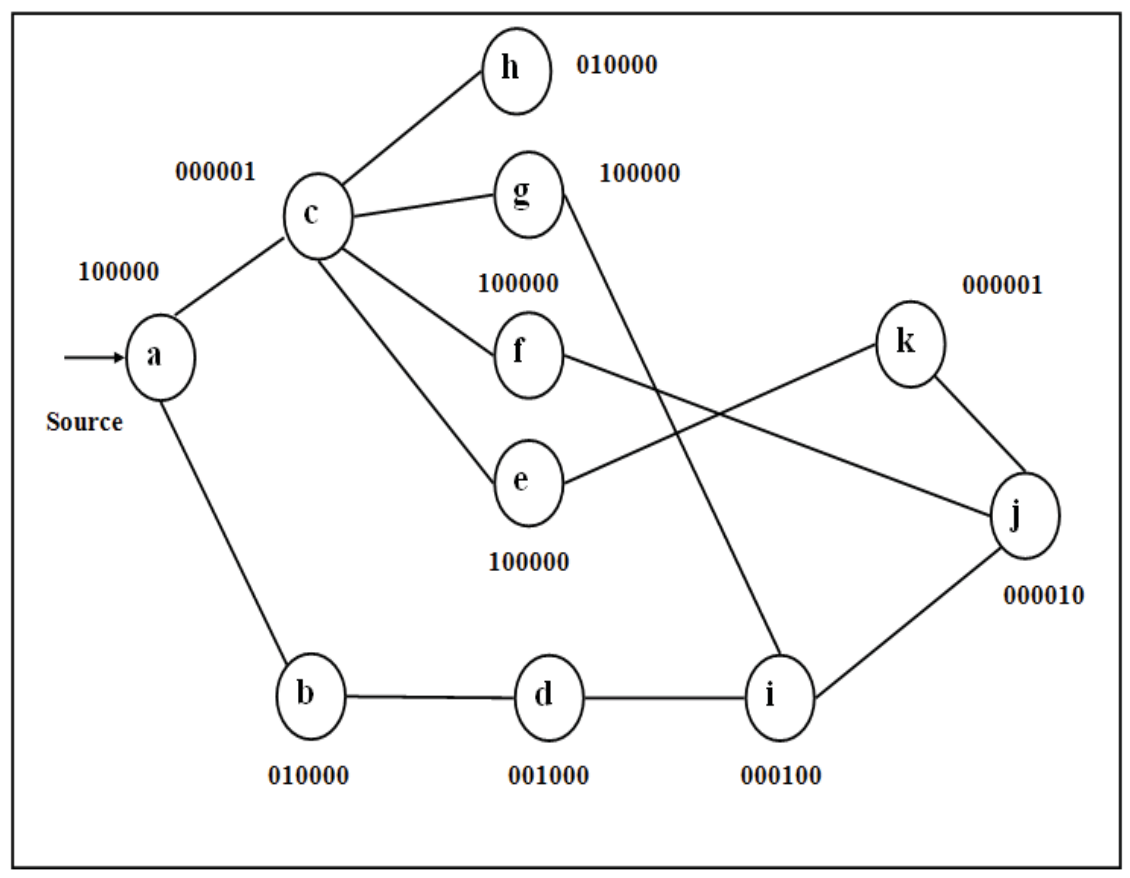

(a)

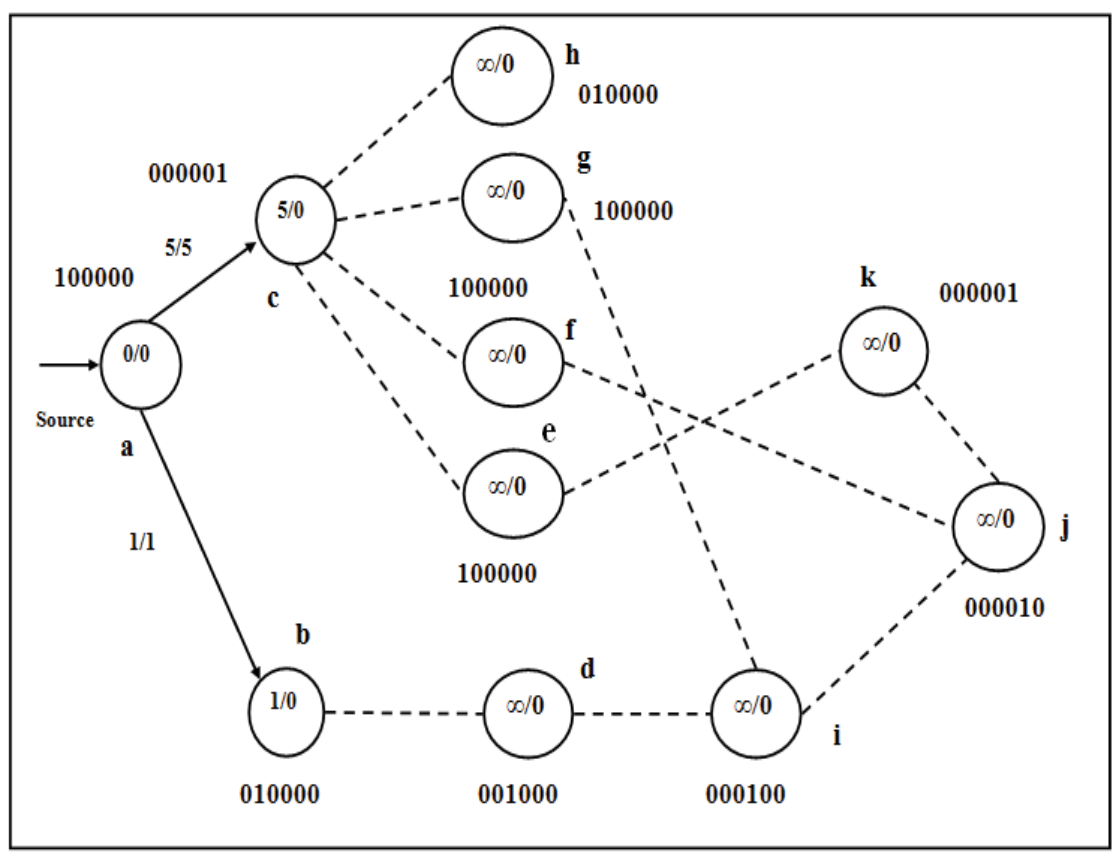

(b)

Figure 7: Construction of $T$ with STIC algorithm with $\operatorname{cost}(T)=15$ 


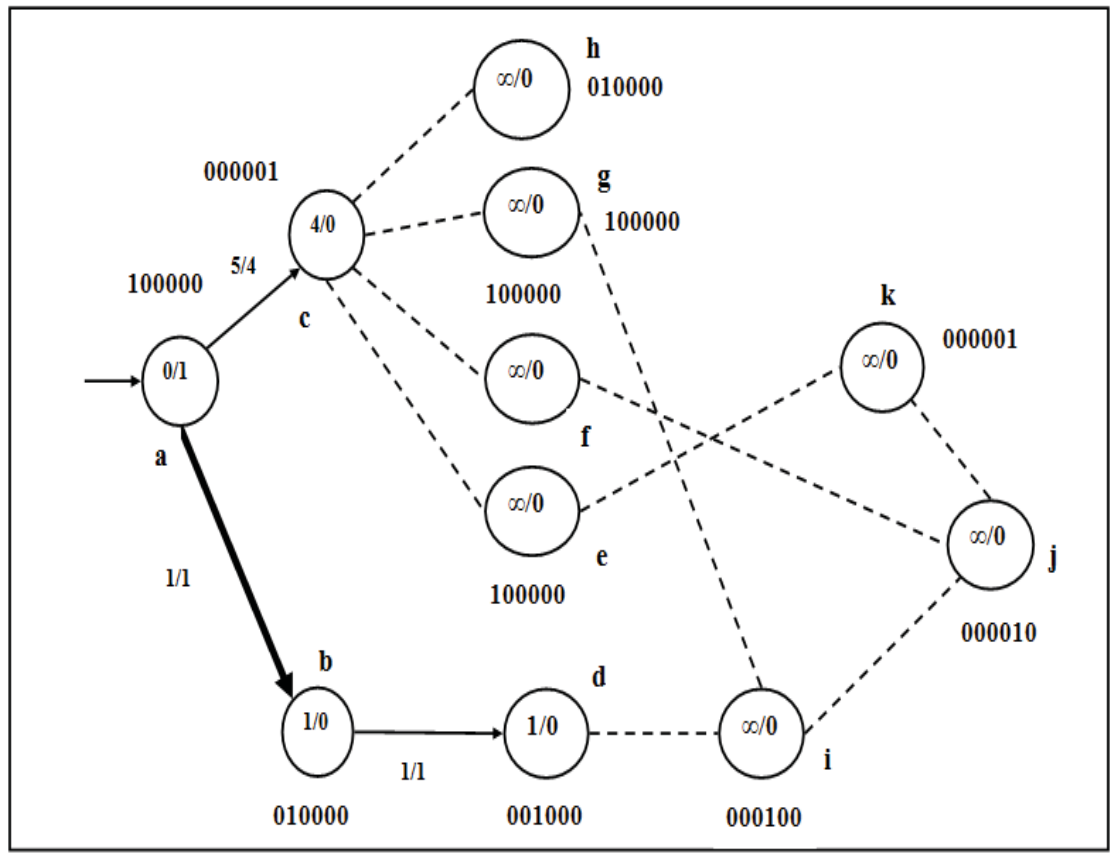

(a)

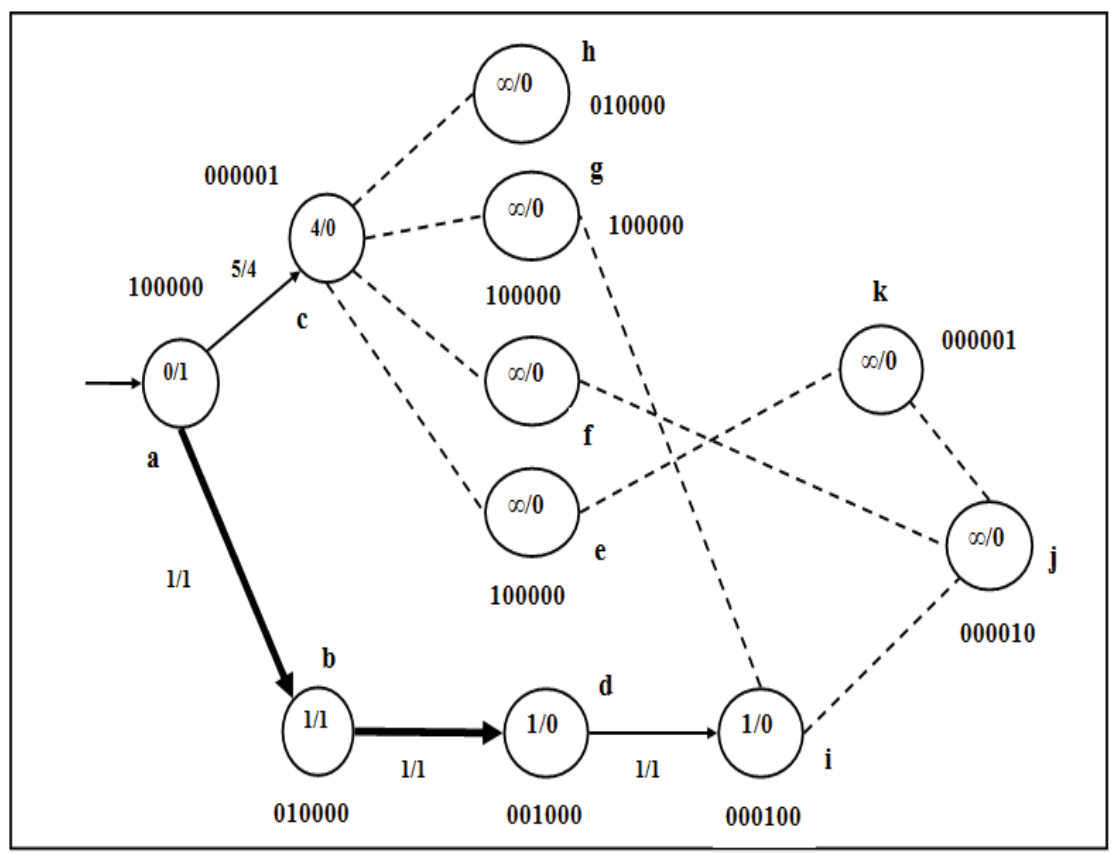

(b)

Figure 8: Construction of $T$ with STIC algorithm with $\operatorname{cost}(T)=15$ 


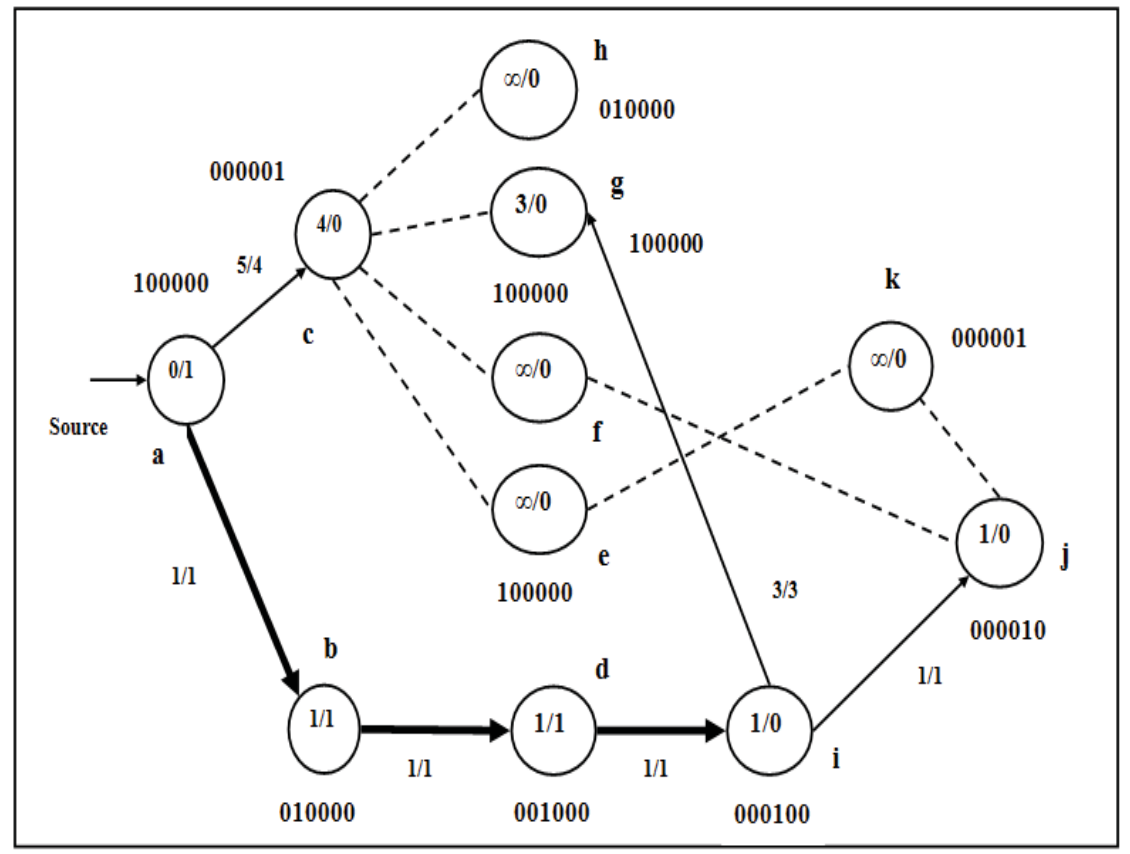

(a)

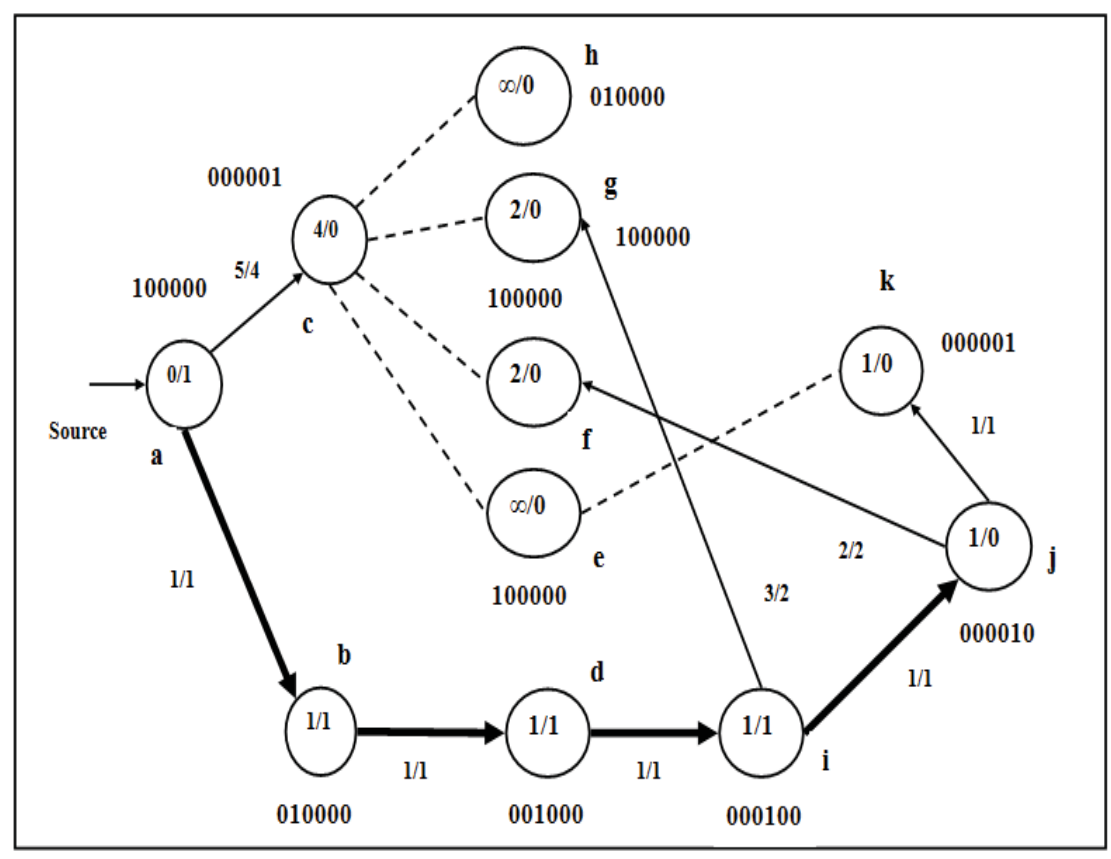

(b)

Figure 9: Construction of $T$ with STIC algorithm with $\operatorname{cost}(T)=15$ 


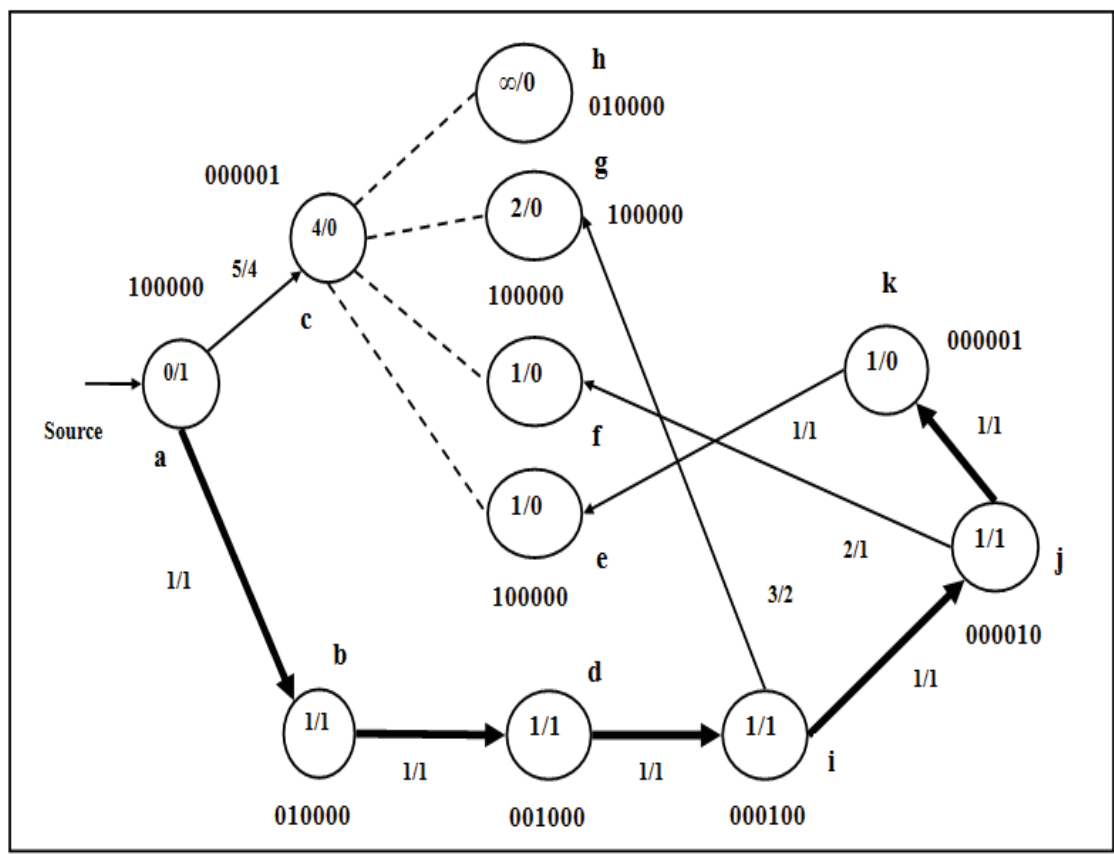

(a)

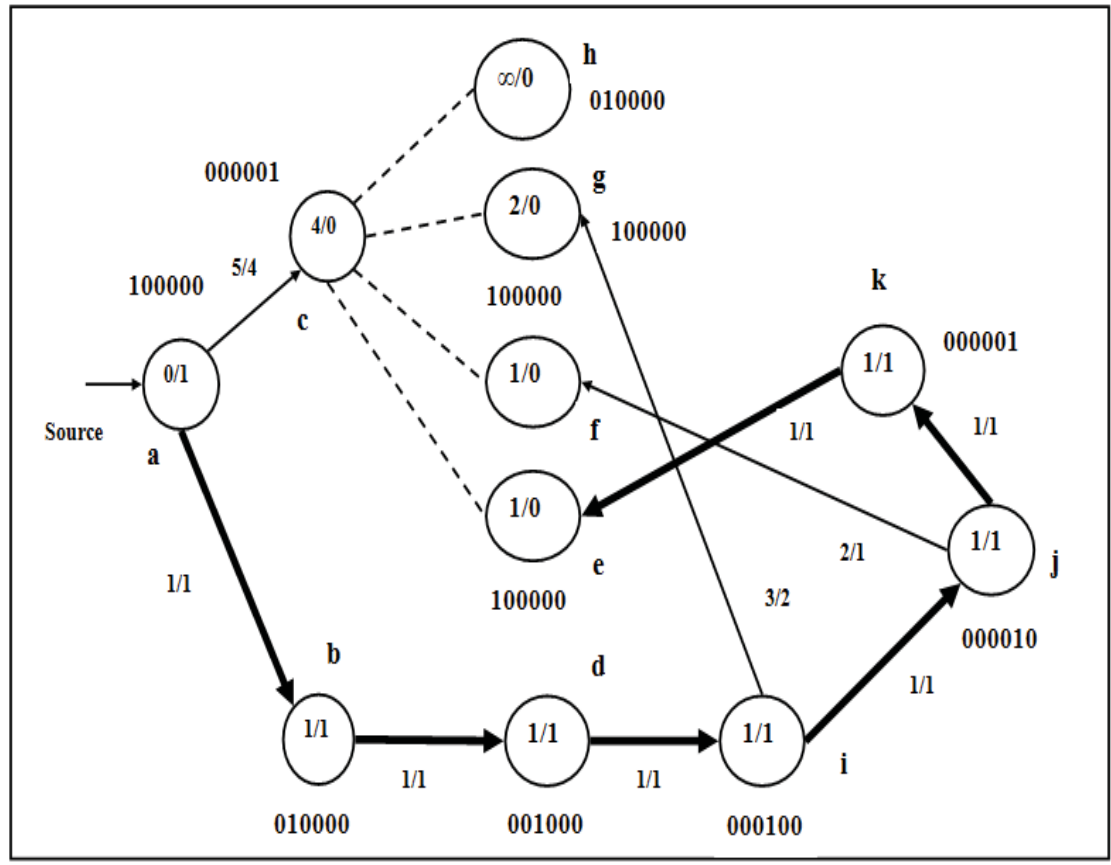

(b)

Figure 10: Construction of $T$ with STIC algorithm with $\operatorname{cost}(T)=15$ 


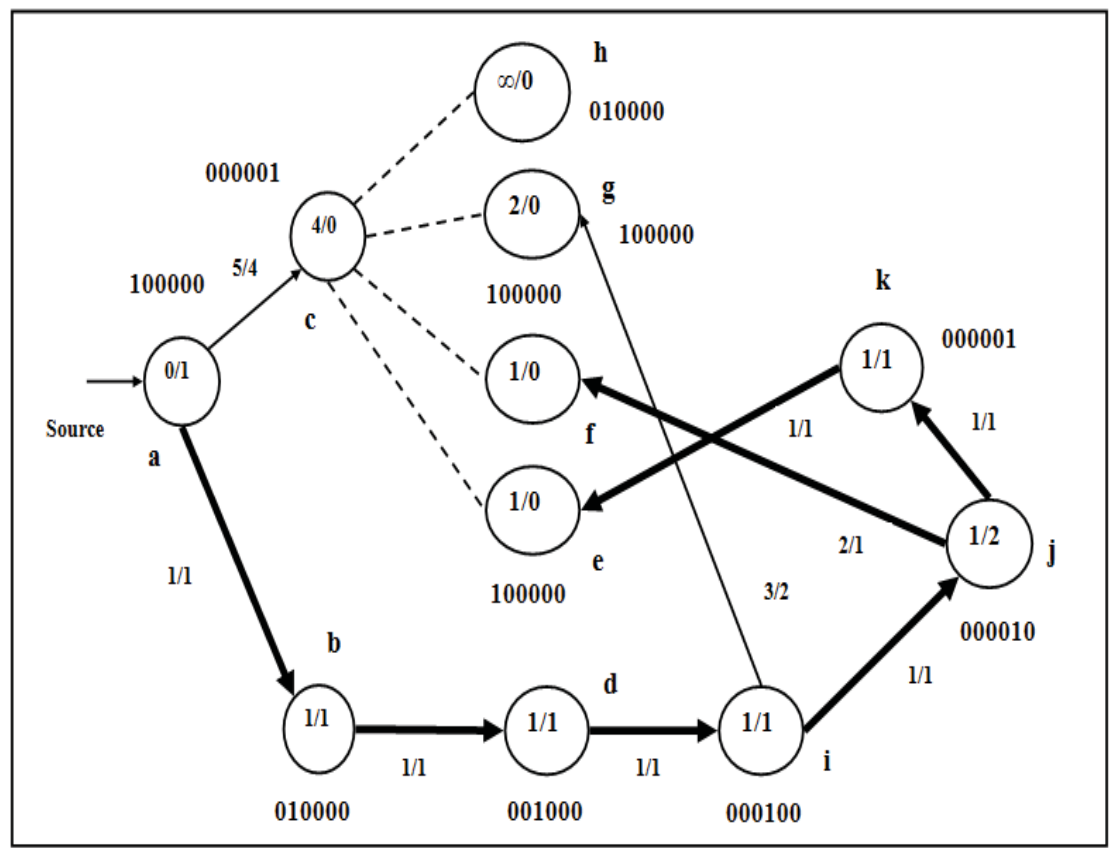

(a)

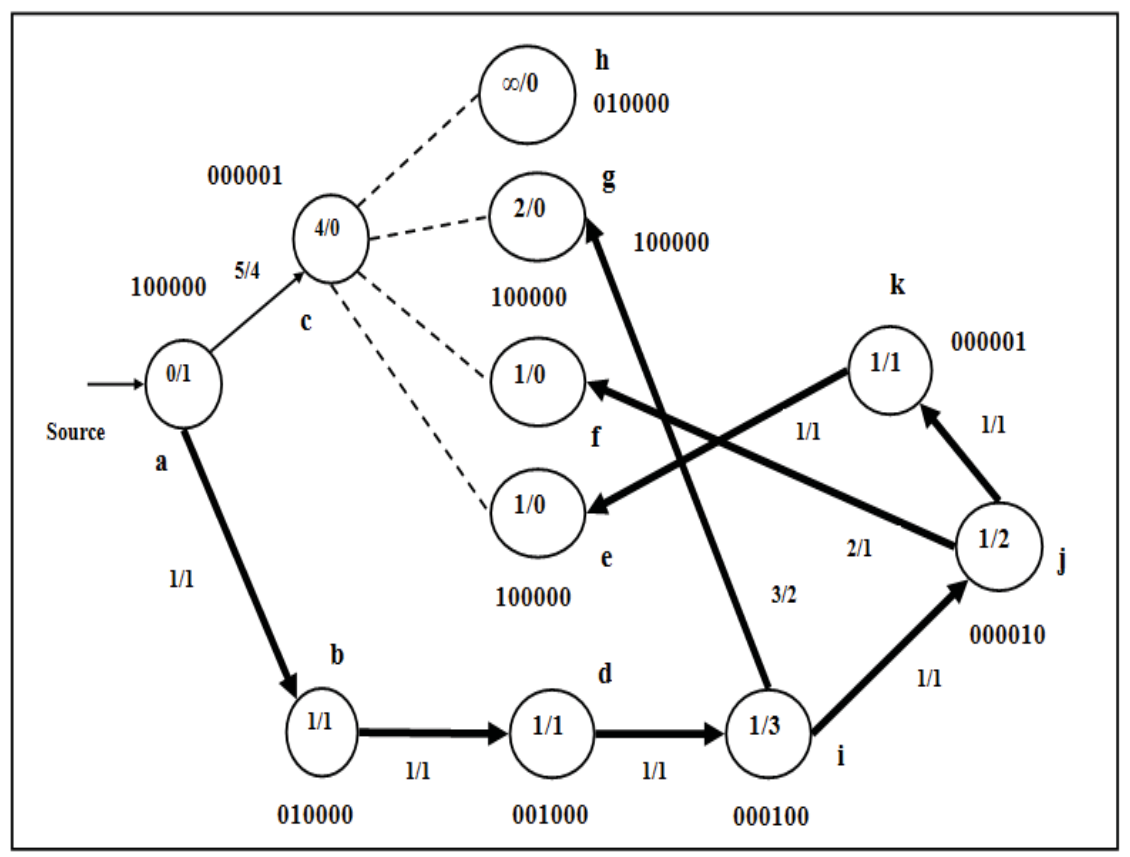

(b)

Figure 11: Construction of $T$ with STIC algorithm with $\operatorname{cost}(T)=15$ 


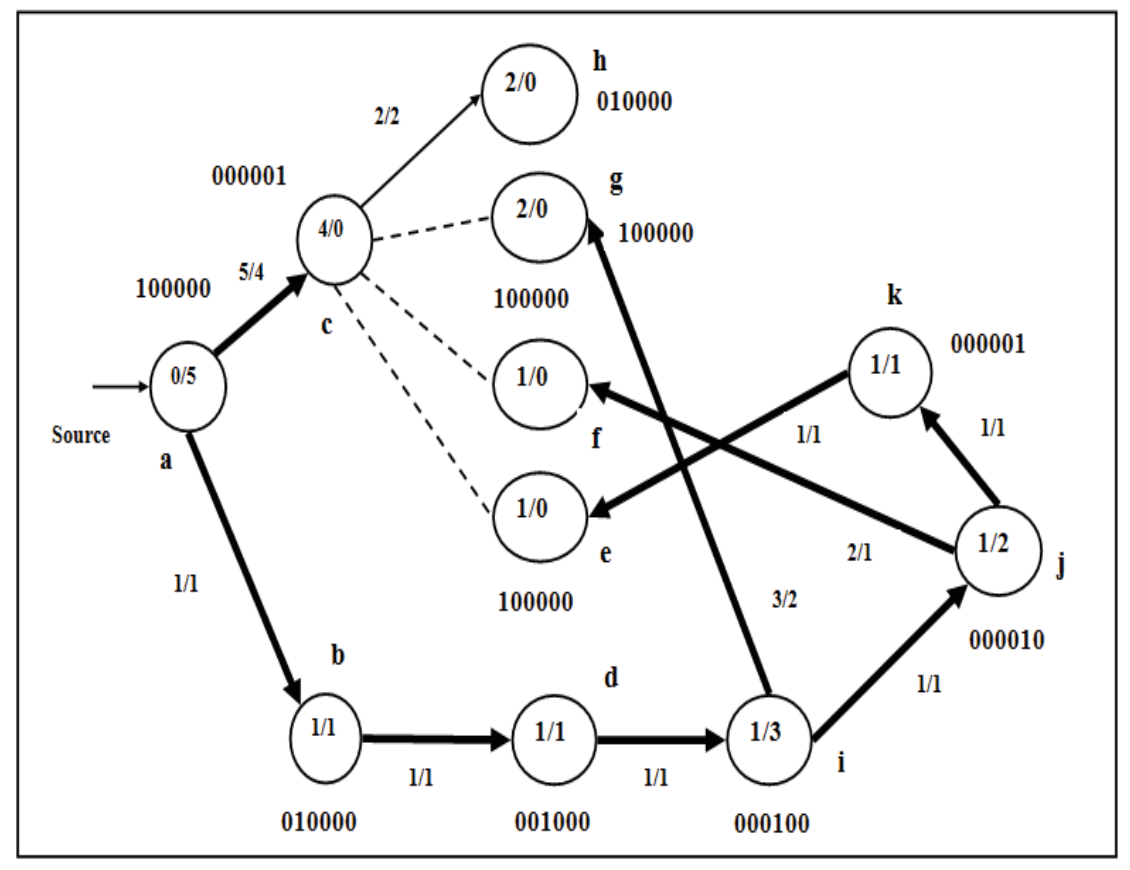

(a)

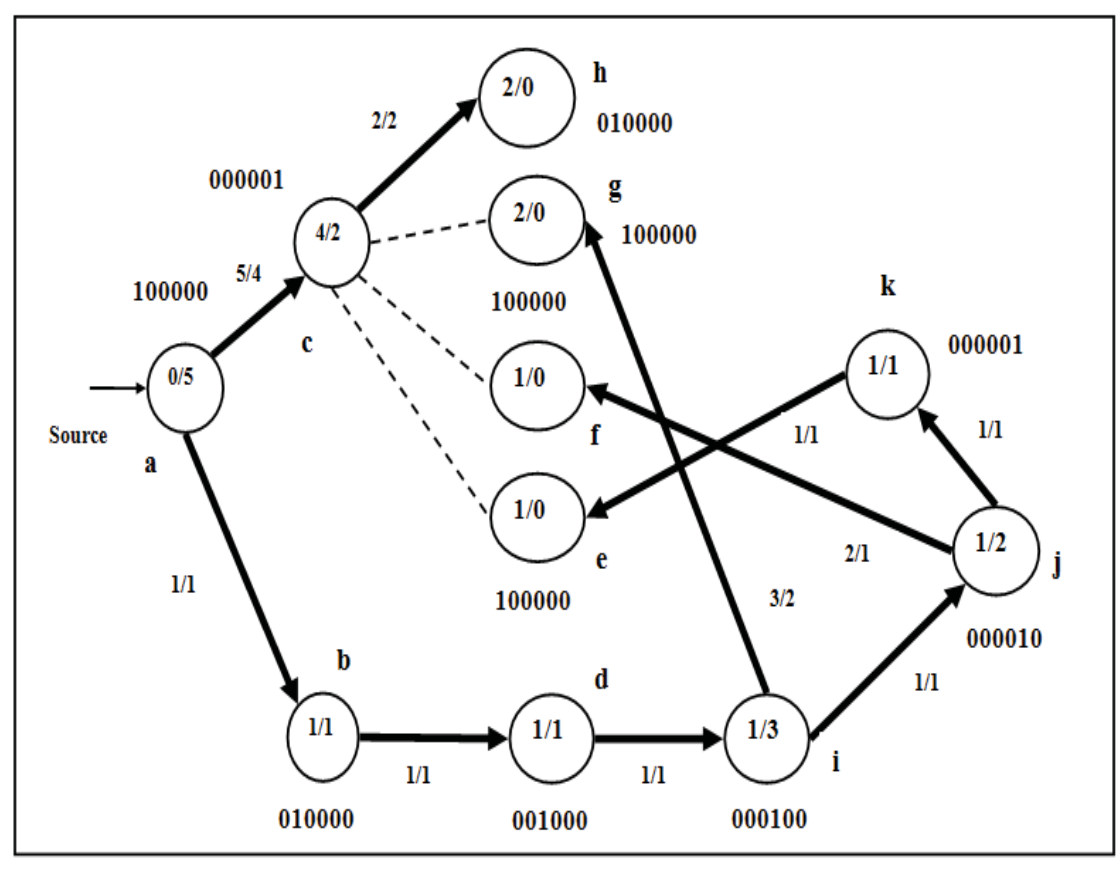

(b)

Figure 12: Construction of $T$ with STIC algorithm with $\operatorname{cost}(T)=15$ 


\subsubsection{MST_Edmonds Algorithm}

This algorithm is based on Edmonds' algorithm for finding the minimum cost spanning tree in a directed graph [10]. We calculate the cost of the edges based on the active time units of the nodes. We consider the wireless sensor network $G=(V, E, M)$ as a directed graph and proceed through the following steps:

1. Weight Calculation: As in the STIC algorithm, the cost of an arc $(u, v)$ is calculated as $w(u, v)=t^{\prime}-t \quad(\bmod n)$, where $t$ and $t^{\prime}$ are the active time units of $u$ and $v$ respectively and the subtraction is done in modulo $n$ arithmetic.

2. Initialization: All the incoming arcs into $s$ are discarded and for every node $u$ other than $s$, its lowest cost incoming arc is selected as its only incoming arc. This set of $N-1$ arcs is denoted by $S$.

3. Contraction of Nodes: If $S$ does not contain any cycle, then $S$ is the desired broadcast tree. Otherwise, for each cycle $C$, all the nodes involved in $C$, are replaced by a pseudo-node $p(C)$ in $G$ and the resulting graph is denoted by $G^{\prime}$. Let $x(C)$ be the cost of the minimum cost arc in $C$. The weight of each arc which enters a node $j$ in $C$ from some node $i$ outside $C$ is modified as $w(i, p(C))=w(i, j)-(w(\operatorname{pred}(j), j)-x(C))$, where $\operatorname{pred}(j)$ is the predecessor of $j$ in $C$. We recursively call the algorithm on $G^{\prime}$.

4. Extraction of Nodes: Given the broadcast tree $S^{\prime}$ that is the output of the recursive call, each pseudo-node $v$ in $S^{\prime}$ is replaced by the nodes involved in the original cycle corresponding to $v$. The arc $(i, v)$ in the tree $S^{\prime}$ corresponds to an arc $(i, x)$ in the original graph $G$ where $x \in C$. We include the arc $(i, x)$ in $S$ and remove the incoming arc into $x$ in the cycle $C$. This ensures that $S$ is a tree.

After constructing broadcast tree $T$, the broadcast schedule is determined and the cost is calculated. The complexity of the algorithm is $O(m \log N)$ for sparse graphs and $O\left(N^{2}\right)$ for dense graphs. The pseudocode of the algorithm is shown in Algorithm 2. 


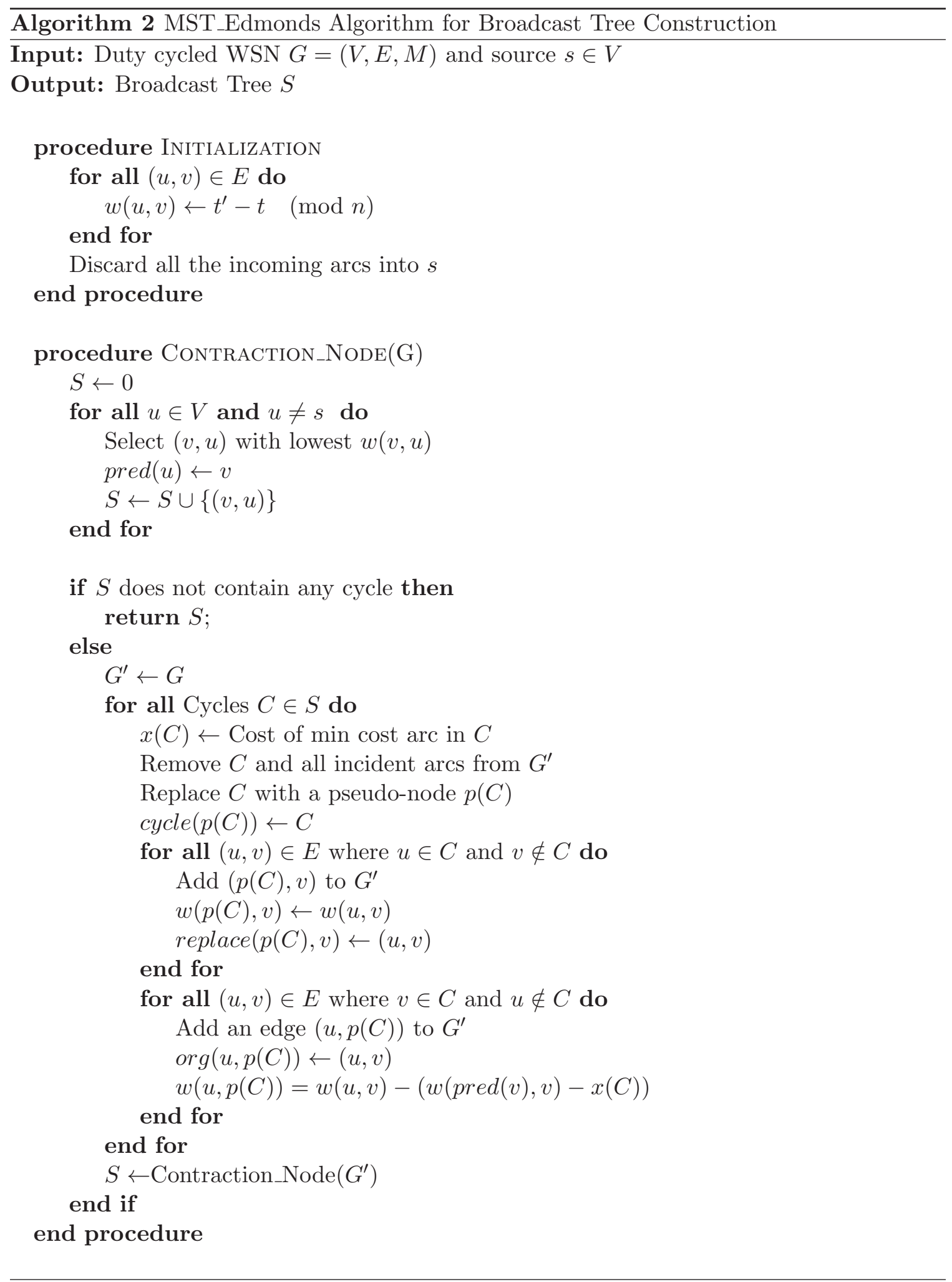




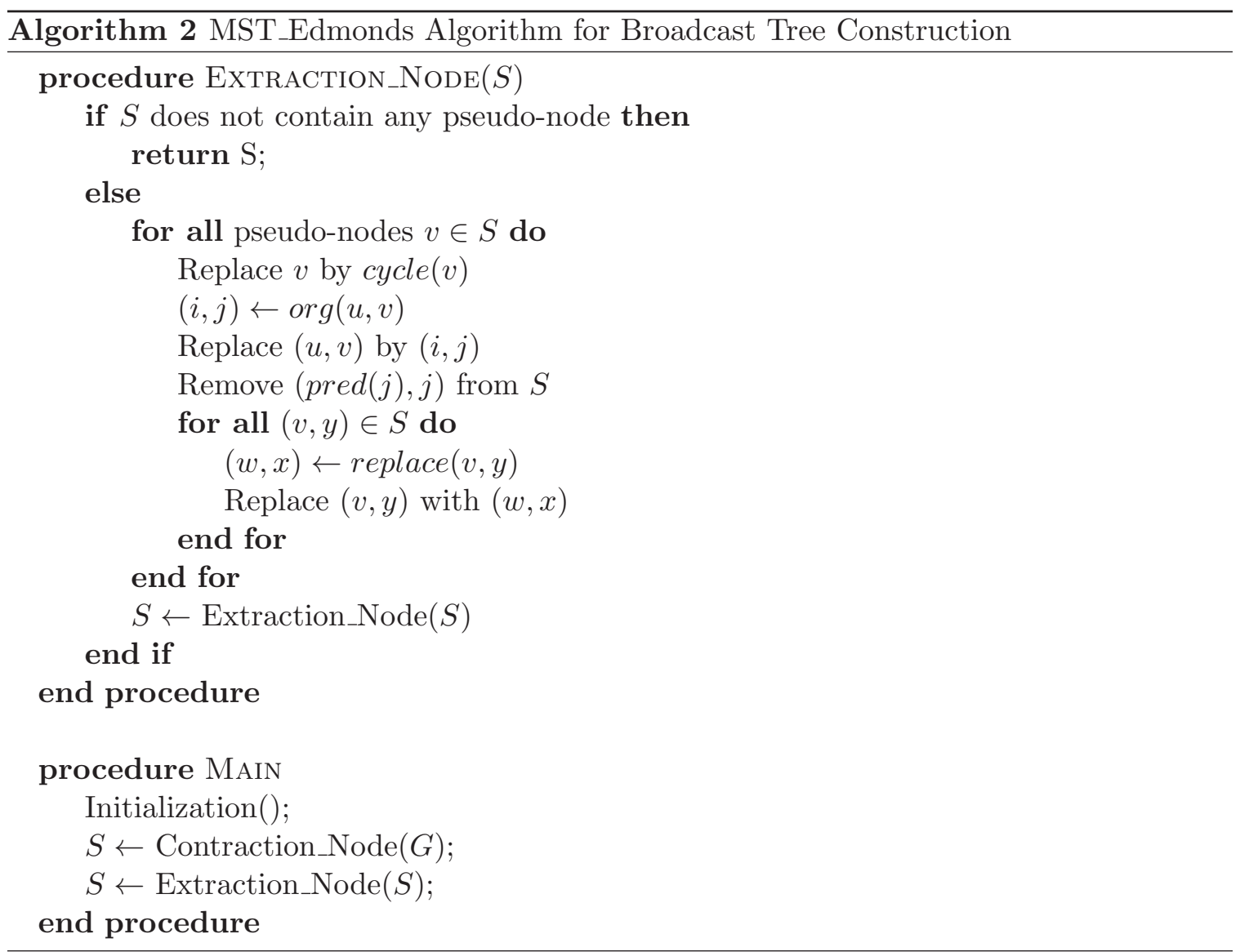

We illustrate the MST_Edmonds algorithm on our example of Figure 6. The directed graph representation of $G$ for this example is shown in Figure 13(a). All the incoming arcs into node $a$ are discarded and for every other node $u \in V$ both the incoming and outgoing arcs are considered. The cost of an $\operatorname{arc}(u, v)$ is calculated as $w(u, v)=t^{\prime}-t \quad(\bmod n)$, where $t$ and $t^{\prime}$ are the active time units of $u$ and $v$ respectively. For an example, node $a$ is active at time unit 1 and node $c$ is active at time unit 6 and thus $w(a, c)=(6-1) \quad(\bmod 6)=5$. During the initialization phase, every node except $a$ selects the lowest weight incoming arc as its only incoming arc. The resulting graph is denoted as $S$ and is shown in Figure 13(b). To distinguish between a cycle and corresponding pseudo-node, we represent a cycle of $k$ nodes $v_{i}, i=1, \ldots, k$ by $\left[v_{1}, v_{2}, \ldots, v_{k}\right]$ and the pseudo-node replacing the cycle is named $\left\{v_{1}, v_{2}, \ldots, v_{k}\right\}$. As shown in Figure $13(\mathrm{~b}), S$ contains a cycle $[c, h]$ and thus it enters into contraction phase. Node $c$ and $h$ are contracted and replaced with pseudo-node $\{c, h\}$. Then $\operatorname{cycle}(\{c, h\})$ is updated to $[c, h]$ and $x(C)$ is set to 2 as it is the minimum weight of the arcs 
in $[c, h]$ and $\operatorname{org}(a,\{c, h\})$ is updated to $(a, c)$ and $w(a,\{c, h\})$ becomes 3 as $w(a,\{c, h\})=$ $w(a, c)-(w(\operatorname{pred}(c), c)-x(C))=5-(4-2)=3$. Again, $\operatorname{org}(g,\{c, h\})$ is updated to $(g, c)$ and $w(g,\{c, h\})$ becomes 3 as $w(g,\{c, h\})=w(g, c)-(w(\operatorname{pred}(c), c)-x(C))=5-(4-2)=3$. Similarly, $\operatorname{org}(f,\{c, h\})$ is updated to $(f, c)$ and $\operatorname{org}(e,\{c, h\})$ is updated to $(e, c)$. The weights $w(f,\{c, h\})$ and $w(e,\{c, h\})$ are updated to 3 . Arc $(c, e) \in G$ is replaced with $(\{c, h\}, e) \in G^{\prime}$ with $w(\{c, h\}, e)=w(c, e)=1$. Similarly, $(c, f)$ and $(c, g)$ is replaced with $(\{c, h\}, f)$ along with $w(\{c, h\}, f)=1$ and $(\{c, h\}, g)$ with $w(\{c, h\}, g)=1$, respectively. Then replace $(\{c, h\}, e)$, replace $(\{c, h\}, f)$ and replace $(\{c, h\}, g)$ are updated to $(c, e),(c, f)$ and $(c, g)$, respectively. The resulting intermediary graph $G^{\prime}$ is shown in Figure 14(a). $S^{\prime}$ is obtained from $G^{\prime}$ by selecting the lowest cost incoming arc for every node $u \in V$ except a. $S^{\prime}$ is shown in Figure $14(\mathrm{~b})$ and as it does not contain any cycle, the algorithm enters into extraction phase.

In the extraction step, pseudo-node $\{c, h\}$ is replaced with cycle $(\{c, h\})=[c, h]$ that is with node $c$ and $h$. Arc $(a,\{c, h\})$ is replaced with $\operatorname{org}(a,\{c, h\})$ that is $(a, c)$ with $\mathrm{w}(a, c)=$ 5. Then $(\operatorname{pred}(c), c)$ that is $(h, c)$ is removed and $(\{c, h\}, e),(\{c, h\}, f)$ and $(\{c, h\}, g)$ are replaced with replace $(\{c, h\}, e)=(c, e)$, replace $(\{c, h\}, f)=(c, f)$ and replace $(\{c, h\}, g)=$ $(c, g)$, respectively. As resulting $S$ does not contain any cycle, $S$ is the broadcast tree $T$ with $\operatorname{cost}(T)=11$. Broadcast tree $T$ is shown in Figure 15. Active time units for each node are shown in ().

\subsubsection{The Sweep Operation}

In this section, we show how to reduce the cost of $T$ constructed by all of the algorithms using a sweep operation which consists of scanning the nodes and making local adjustments to the tree. A similar idea was used in [51] to adjust transmission powers of nodes in a previously constructed solution.

The main idea of our sweep operation is to scan the nodes of $T$ in some pre-specified order. When a node $u$ is scanned, we check for each node $v$ that can be reached from $u$ without increasing the active time of $u$, whether making $u$ the parent of $v$ will decrease the cost of the tree. To avoid creating a loop, this process excludes the nodes between 


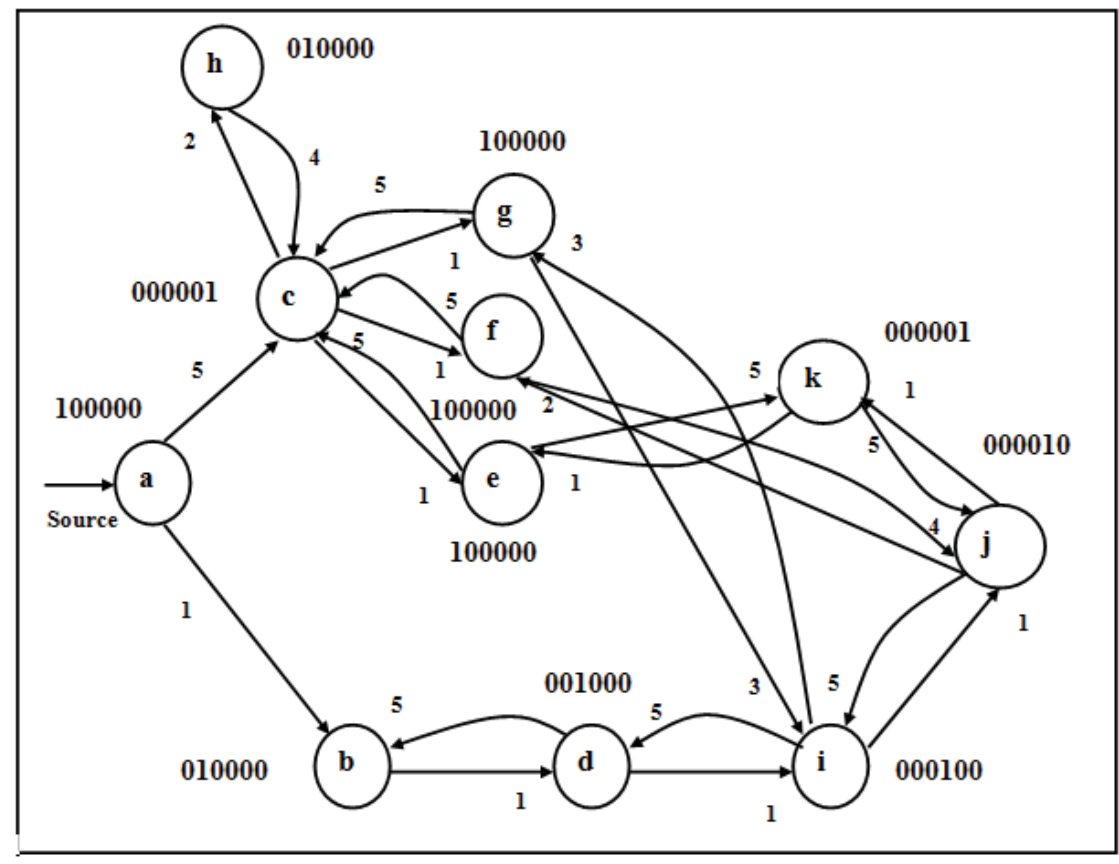

(a) Directed graph representation of $G=(V, E, M)$

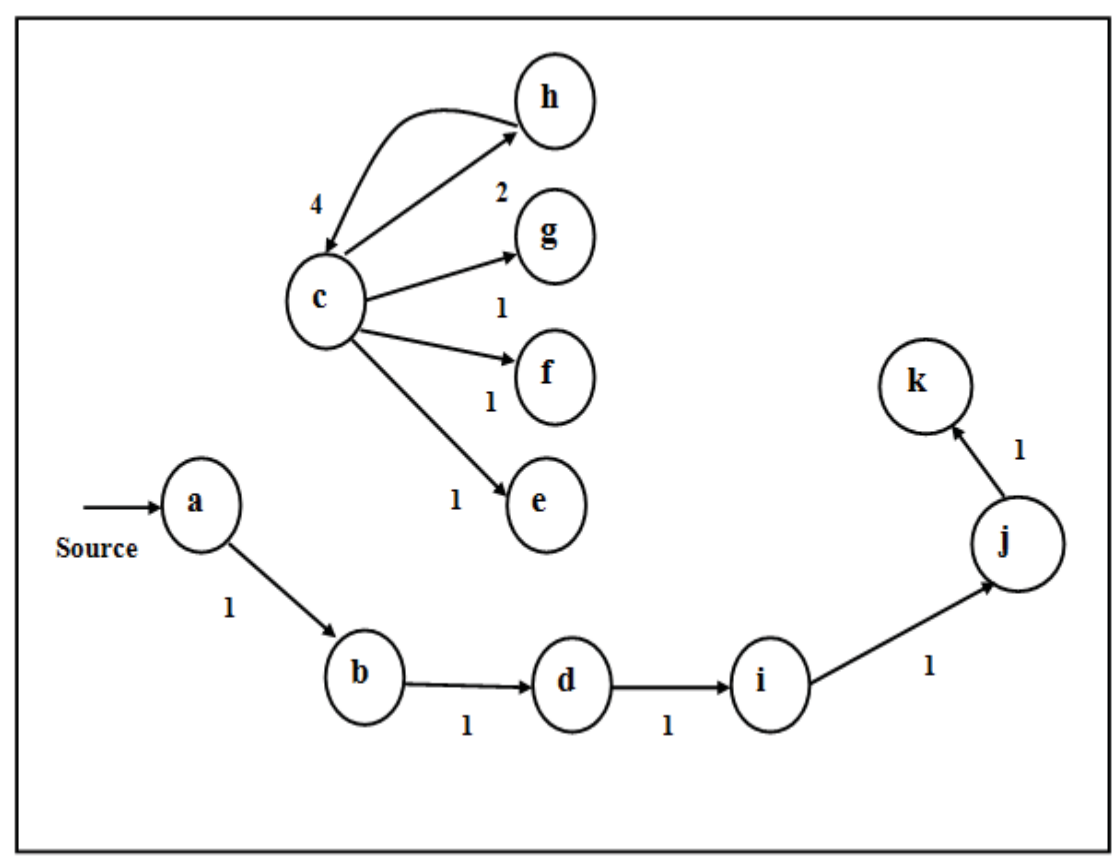

(b) $S$ contains a cycle $[c, h]$

Figure 13: Construction of $T$ with MST_Edmonds algorithm with $\operatorname{cost}(T)=11$ 




(a) Intermediate graph $G^{\prime}$ after contracting node $c$ and $h$

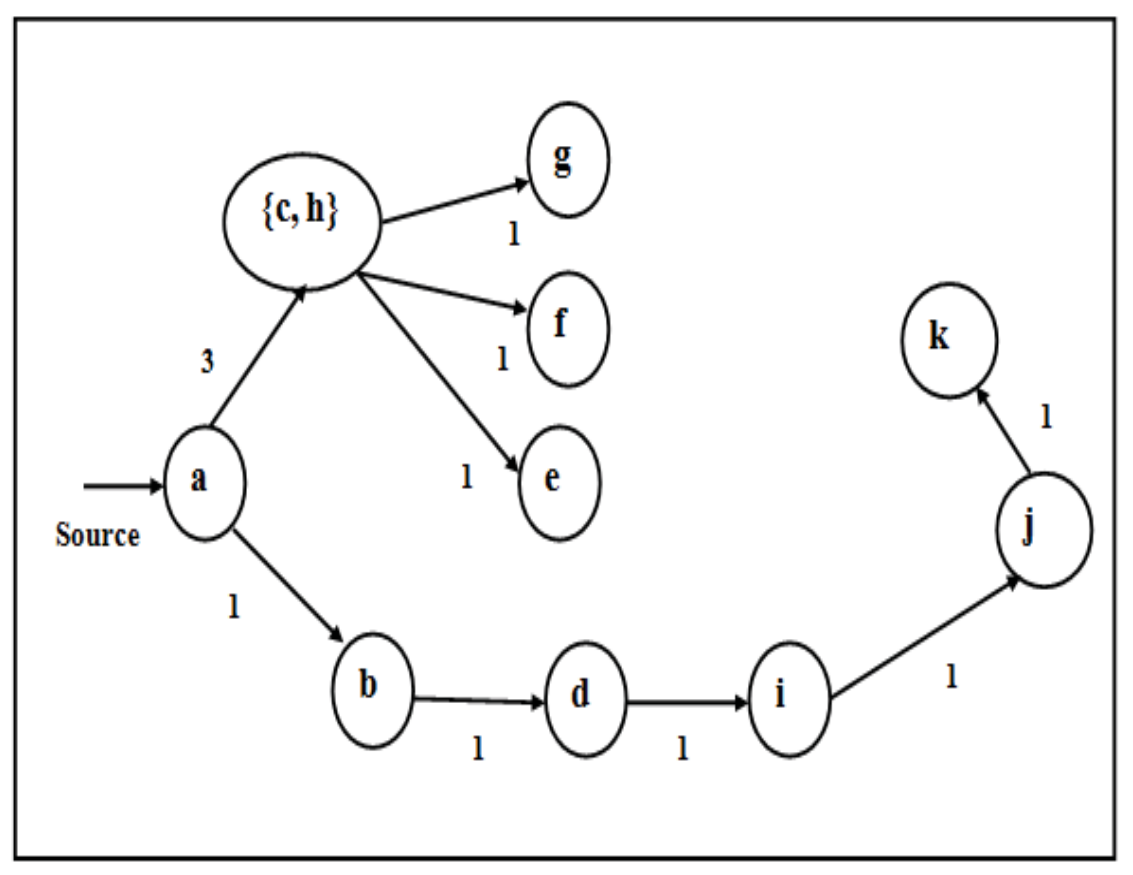

(b) $S^{\prime}$ obtained from $G^{\prime}$

Figure 14: Construction of $T$ with MST_Edmonds algorithm with $\operatorname{cost}(T)=11$ 


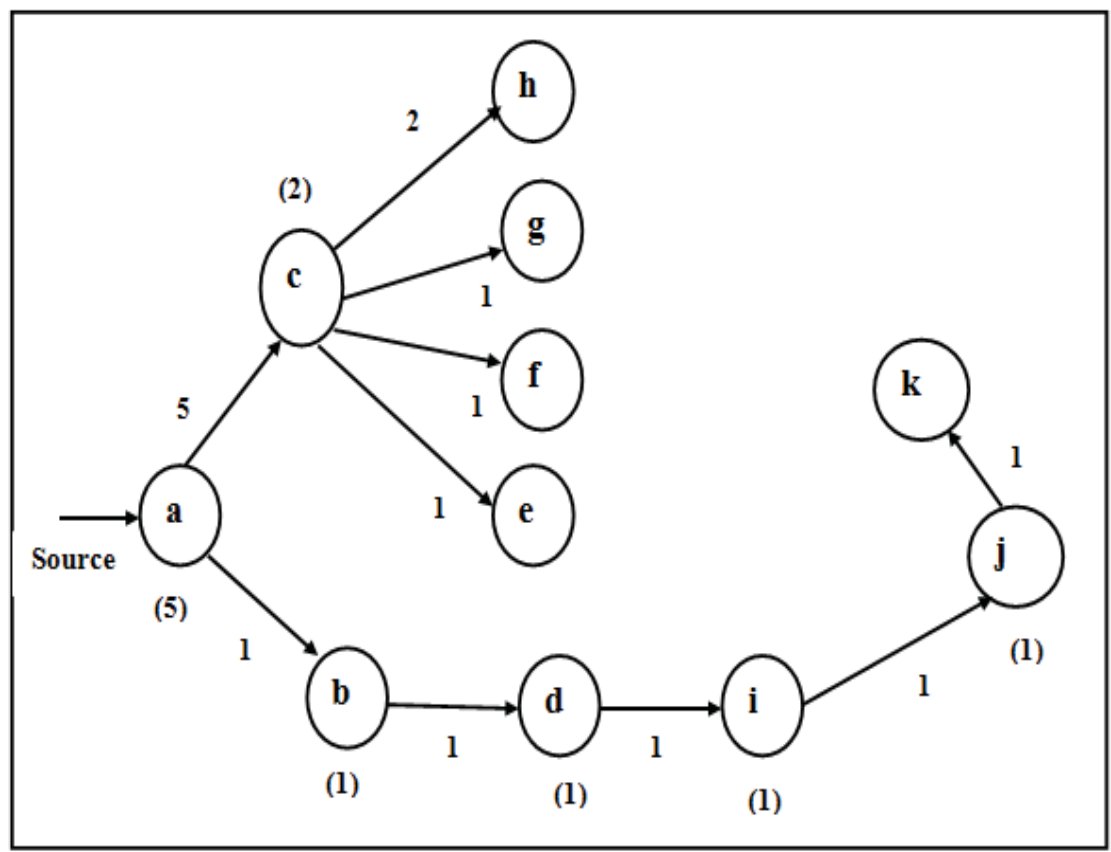

Figure 15: $S$ as well as broadcast tree $T$ obtained after extraction phase

$u$ and $s$ in $T$. Clearly, this process may decrease the cost of $T$ but never increases its cost. The complexity of the sweep operation is $O\left(N^{2}\right)$, where $N$ is the number of nodes. The sweep operation can be applied multiple times, but experiments show that significant improvement is achieved at the first application and repeating sweep rarely improves the result. The order in which nodes are scanned can have an effect on the result. We consider five different node orderings:

- Increasing order of node ID (sweep): Nodes are arranged in the ascending order of their IDs and scanned in this order.

- BFS order of nodes (bfs): We start with $s$ and scan the nodes at distance $k$ from $s$ before the nodes at $k+1$.

- Bottom up order of nodes (buo): We start with nodes at the next to the bottom level and gradually move toward $s$.

- Decreasing order of active time units (dec): Nodes are sorted in descending order of the active time units associated with them and scanned in this order during the sweep 
operation.

- Increasing order of active time units (inc): Nodes are sorted in ascending order of active time units associated with them and then scanned accordingly in the sweep operation.

A sweep operation using any of the above orderings can be performed on the broadcast tree produced by any algorithm. We denote a broadcast tree algorithm augmented by a particular type of sweep by Algorithm_sweeptype. For example, the MST_Edmonds algorithm augmented by a sweep in order of increasing order of active time units (inc) is called MST_Edmonds_inc.

We illustrate the sweep operation on $T$ generated by STIC algorithm as shown in Figure 16(a). Each node $u$ is labeled inside the circle with the additional active time units for which it is awake. We consider the increasing node ID version of the sweep operation(STIC_sweep) that is, nodes are scanned in increasing order of node ID starting with node $a$. Node $a$ and $b$ do not cause any improvement to $\operatorname{cost}(T)$. Node $c$ has to stay awake 2 time units to send messages to node $h$ as it is the only parent of $h$. During this active time period, it can also transmit messages to node $e, f$ and $g$ (as shown in Figure 16(a), e, $f$ and $g$ are shaded to indicate that they are in transmission range of node $c$ ). By making $c$ the parent of $e, f$ and $g$ we can eliminate 1 active time unit from $k$ (the previous parent of $e$ ), 1 time unit from $j$ (the previous parent of $f$ ) and 2 time units from $i$ (previous parent of $g$ ). Thus it reduces 4 time units without increasing the number of time units that node $c$ needs to be active. No other nodes of $T$ cause any improvement of $\operatorname{cost}(T)$. Thus the sweep operation improves the cost by 4 time units. The cost of the final broadcast tree $T$ is 11 in this example. 


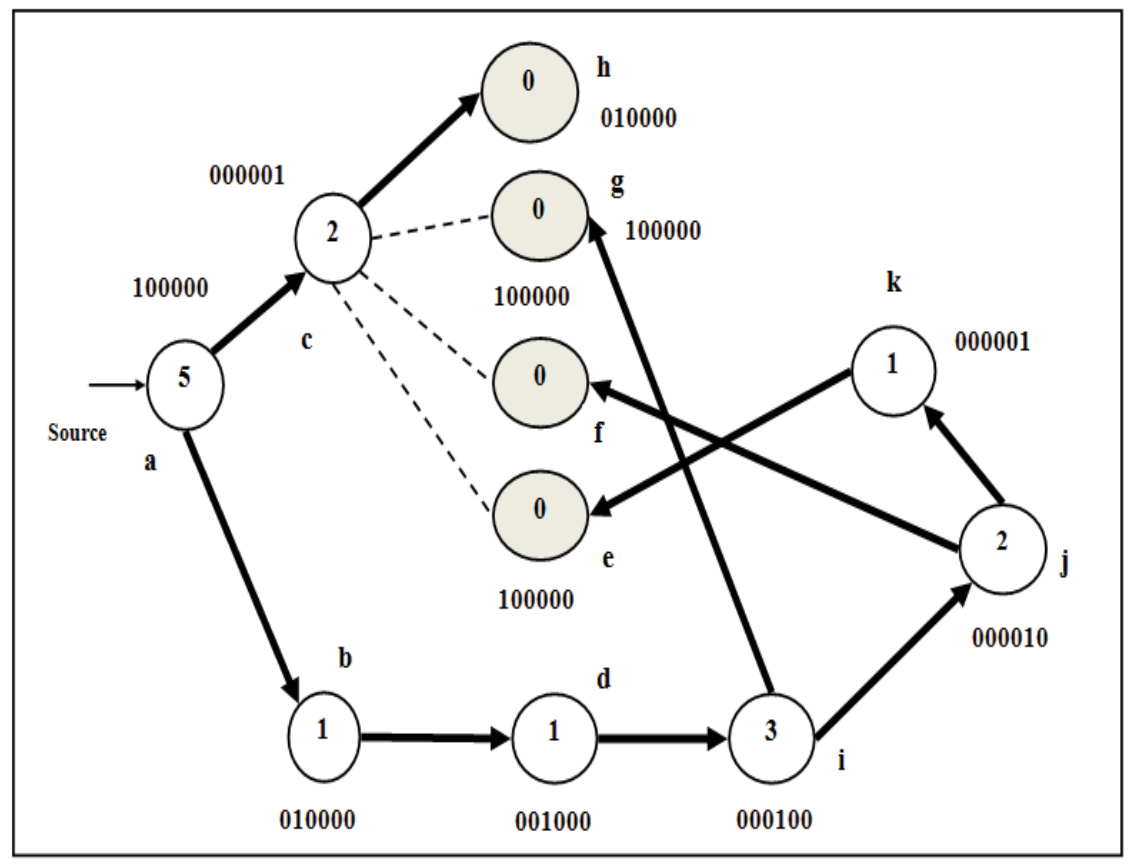

(a) Broadcast tree $T$ with $\operatorname{cost}(T)=15$ constructed by STIC

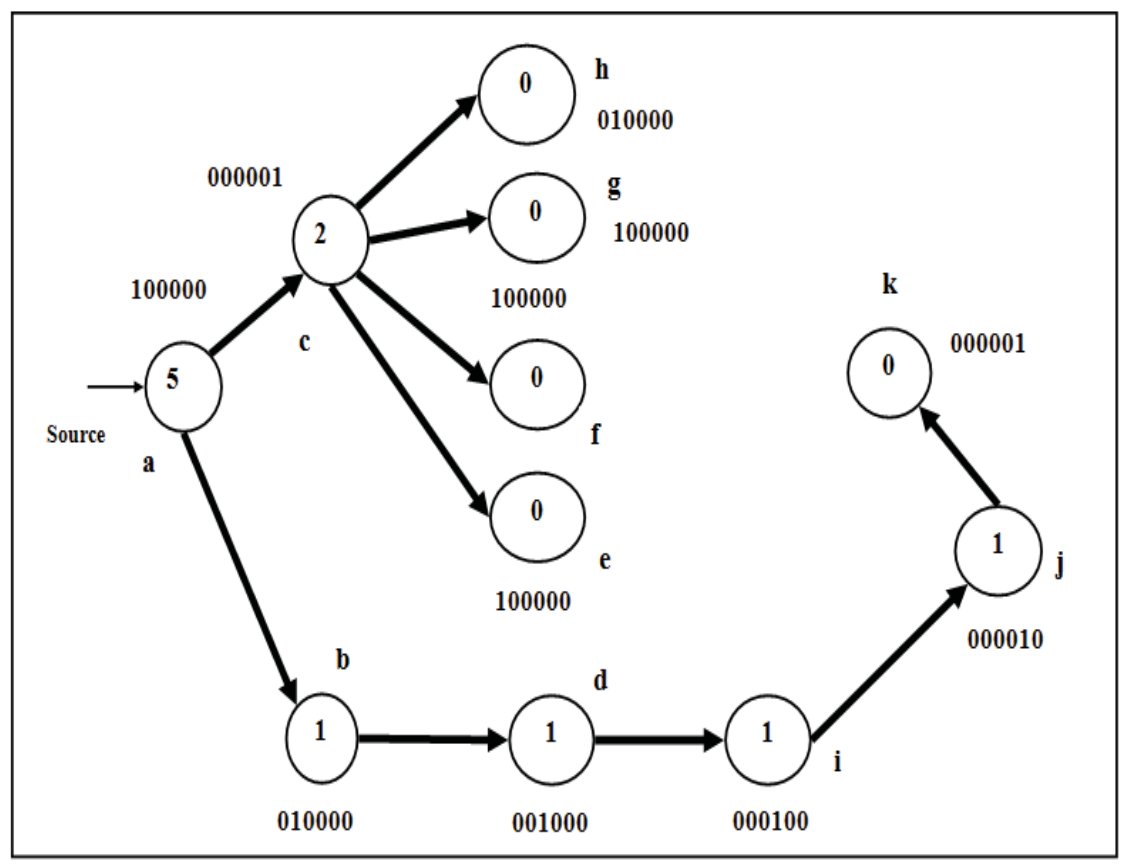

(b) Broadcast tree $T$ with $\operatorname{cost}(T)=11$ after the Sweep Operation

Figure 16: Sweep Operation on $T$ 


\section{Chapter 4}

\section{Experimental Results}

In this chapter, we present the performance analysis of our proposed algorithms. We conducted extensive simulations in order to compare the performance of the proposed algorithms with two existing algorithms for broadcasting named CSCA [18] and SDT [22] described in Chapter 2. The main performance metrics used were the average number of additional active time units per node, the distribution of energy usage over the nodes, the number of node transmissions and the maximum and average delay of the broadcast operation.

In all our simulations, we used Java Platform (JDK 6 update 14). We generated networks where the nodes were distributed uniformly at random in a geographic area of 200 by 200 . We considered networks with the number of nodes 50, 100, 150, 200, 250, 300, 350 and 400. The number of nodes in a network is denoted by $N$. For each value of $N$, we generated 1000 connected graphs and they were stored in a file. For each value of $N$, the same graphs were used across all simulations. All the results in this chapter are averaged over 1000 topologies. We used the density measures of 8, 10 and 12. Given the node density measure we calculated the transmission range of a node for each value of $N$. Two nodes are adjacent if and only if their Euclidean distance is less than or equal to the transmission range. For each topology, a source node which is responsible for initiating the broadcast message is selected at random. Every node in the network is accompanied with a wakeup schedule which is a binary array of fixed length. We used the schedule length sch_len of 5, 10, 15 


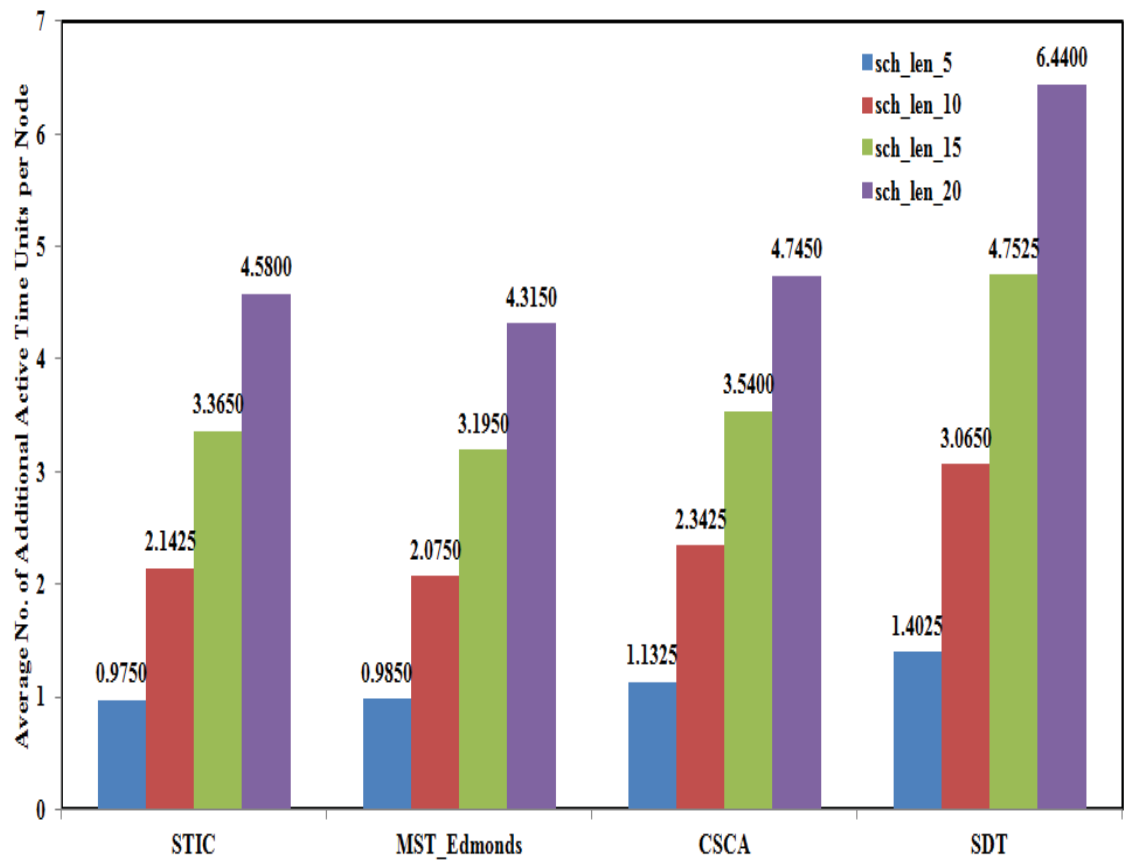

Figure 17: Average no. of additional active time units per node at node density 8 for all values of sch_len and $N=400$

and 20 in our experiments and assigned a single randomly chosen element of the array to be 1 while other elements are assigned to be 0 . This corresponds to a node being active for a single unit of time during the schedule.

\subsection{Performance Comparison of all Algorithms without Sweep Operation}

In this section we analyze the performance of our algorithms, STIC and MST_Edmonds as well as CSCA [18] and SDT [22] without applying the sweep operation. The results are described in the following subsections.

\subsubsection{Average Number of Additional Active Time Units per Node}

For each algorithm we determined the total additional active time units the nodes of a network need to be active in order to carry out the broadcast operation. This value excludes the scheduled active time slot for every node. The total value is then divided by the number 


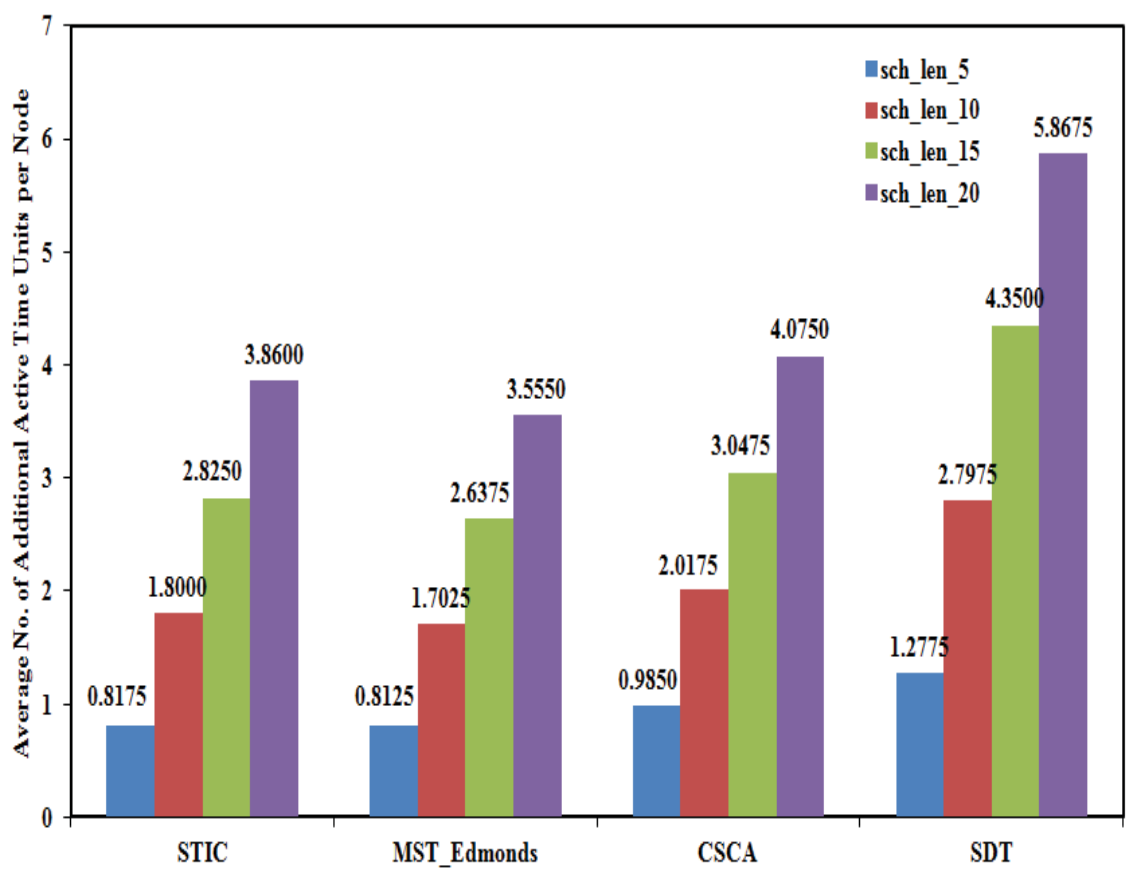

(a) node density 10

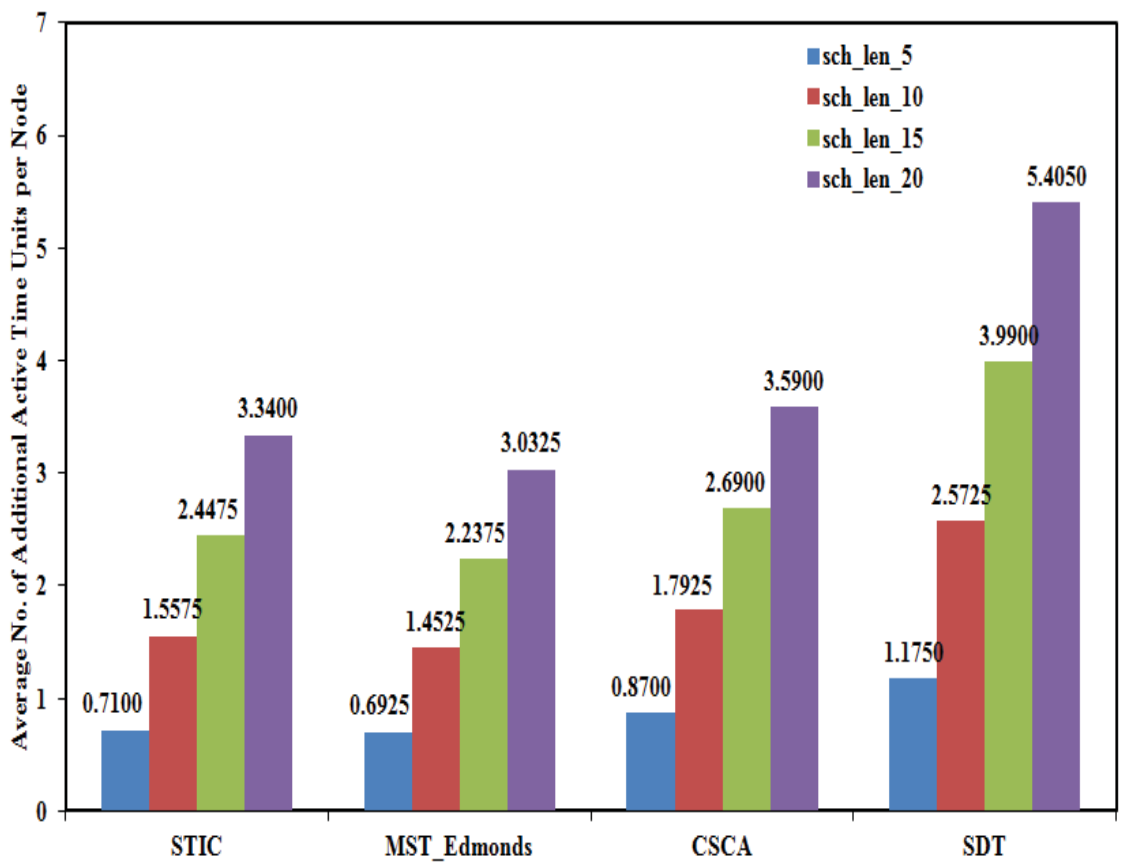

(b) node density 12

Figure 18: Average no. of additional active time units per node for all values of sch_len and $N=400$ 
Table 1: Average Additional Active Time Units per Node with respect to MST_Edmonds for $N=400$ and sch_len $=20$

\begin{tabular}{|c|c|c|c|c|}
\hline Node Density & STIC & MST_Edmonds & CSCA & SDT \\
\hline \hline 8 & 1.06 & 1.00 & 1.10 & 1.49 \\
\hline 10 & 1.09 & 1.00 & 1.15 & 1.65 \\
\hline 12 & 1.10 & 1.00 & 1.18 & 1.78 \\
\hline
\end{tabular}

of nodes in a network to obtain the average number of additional active time units per node. Both of our proposed algorithms STIC and MST_Edmonds reduce the average number of additional active time units per node as compared to CSCA and SDT, while MST_Edmonds produces a slightly better result. The SDT algorithm shows the worst performance among all the algorithms. Our results are illustrated in Figures 17 to 18 for $N=400$. As can be seen from Figure 17, at node density 8 for sch_len=20, the average additional active time units per node for STIC and MST_Edmonds are 4.5800 and 4.3150, respectively which in turn indicate that on average, a node has to stay awake additional time which is about $23 \%$ and $22 \%$ of the schedule for the STIC algorithm and for the MST_Edmonds algorithm, respectively. On the other hand, CSCA and SDT cause a node to be alive for about $24 \%$ and $32 \%$ of the schedule, respectively for the same value of sch_len. All the algorithms reduce the additional active time units per node to a greater extent with the higher values of node density. For example, as depicted in Figure 18(b), at node density 12 for sch_len=20, the average additional active time units per node for STIC, MST_Edmonds, CSCA and SDT are 3.3400, 3.0325, 3.5900 and 5.4050, respectively. That is at node density 12 a node needs to be active only about $17 \%$ and $15 \%$ of the schedule for STIC and MST_Edmonds, respectively. A node has to stay active for about $18 \%$ of the schedule for CSCA while SDT causes a node to be active for about $27 \%$ of the schedule. Both CSCA and SDT produce larger percentage of additional active time units per node compared to MST_Edmonds and STIC and these percentages are gradually increased with higher values of node density. The normalized results with respect to MST_Edmonds for $N=400$ and sch_len=20 are shown in Table 1. For example, at node density=12, on average, STIC produces about $10 \%$ more additional active time units compared to MST_Edmonds while CSCA produces 




Figure 19: Distribution of Energy Usage at node density $=12$ when $N=400$ and sch_len $=$ 15

about $18 \%$ and SDT generates about $78 \%$ more additional active time units compared to MST_Edmonds.

\subsubsection{Energy Distribution}

Another performance measure of broadcast algorithms is the distribution of energy usage. Even if the average energy spent by nodes is reasonable, if there are many nodes with high energy expenditure, then the network can be disconnected or become inoperational. We determined the percentage of nodes that are active more than or equal to $t$ for all values of $t$ in the schedule. In this section, we describe our results of energy usage with respect to $N=400$ and sch_len=15 as the results for other values of sch_len are similar. We present the results for node density 8, 10 in Tables 2 and 3, respectively and node density 12 in Table 4 as well as in Figure 19. As shown in Table 4, at node density 12, about $13.27 \%$ and $23.28 \%$ of nodes have active time units of 14 for the CSCA and SDT algorithms, respectively. On the other hand, STIC produces about $8.02 \%$ nodes with active time units of 14 while MST_Edmonds generates about $2.20 \%$ of nodes with active time units of 14 . 
Table 2: Distribution of Energy Usage at node density 8 when $N=400$ and sch_len=15

\begin{tabular}{|c|c|c|c|c|}
\hline Active Time Units & STIC & MST_Edmonds & CSCA & SDT \\
\hline \hline 0 & 100.00000 & 100.00000 & 100.00000 & 100.00000 \\
\hline 1 & 37.45375 & 54.50875 & 37.81975 & 40.89400 \\
\hline 2 & 28.11625 & 29.19375 & 31.92225 & 37.88975 \\
\hline 3 & 25.52300 & 24.79075 & 28.37175 & 35.96775 \\
\hline 4 & 24.81825 & 23.39550 & 26.30975 & 34.86325 \\
\hline 5 & 24.59300 & 22.83925 & 25.14850 & 34.28600 \\
\hline 6 & 24.47625 & 22.55425 & 24.53900 & 33.99200 \\
\hline 7 & 24.37725 & 22.34225 & 24.19575 & 33.79875 \\
\hline 8 & 24.24950 & 22.09725 & 23.98600 & 33.67450 \\
\hline 9 & 24.00000 & 21.69450 & 23.80925 & 33.54725 \\
\hline 10 & 23.52275 & 20.94850 & 23.59575 & 33.34450 \\
\hline 11 & 22.62775 & 19.55375 & 23.23825 & 32.96025 \\
\hline 12 & 20.93925 & 16.85025 & 22.50150 & 32.19600 \\
\hline 13 & 18.08875 & 12.35825 & 20.97300 & 30.67075 \\
\hline 14 & 13.91800 & 6.59100 & 17.59275 & 27.30050 \\
\hline
\end{tabular}

Thus, the MST_Edmonds algorithm achieves significant improvement in reducing number of nodes with highest possible active time units. At node density 12, STIC produces about 3.65 times more nodes with active time units 14 as compared to MST_Edmonds. On the other hand, CSCA generates about 6.04 times and SDT produces about 10.60 times more nodes with active time units 14 than that of the MST_Edmonds algorithm.

As shown in Table 4 , at node density 12 , about $17.78 \%, 15.76 \%, 18.25 \%$ and $28.43 \%$ of nodes have active time units of 7 for the STIC, MST_Edmonds, CSCA and SDT algorithms, respectively. CSCA, SDT and STIC produce about 1.16, 1.8 and 1.13 times more nodes with active time units of 7, respectively compared to MST_Edmonds. STIC exhibits almost identical performance to CSCA at node density 8 for active time units of 7 but its performance improves with higher node densities and at node density 12, CSCA generates about 1.03 times more nodes than the STIC algorithm.

For all node densities at $N=400$ with sch_len $=15$, the percentage of nodes with active time units 14 generated by all the algorithms is divided by the percentage of nodes with active time units 14 for MST_Edmonds and the result is presented in Table 5. A similar result for the percentage of nodes with active time units of 7 for all algorithms are shown in 
Table 3: Distribution of Energy Usage at node density 10 when $N=400$ and sch_len=15

\begin{tabular}{|c|c|c|c|c|}
\hline Active Time Units & STIC & MST_Edmonds & CSCA & SDT \\
\hline \hline 0 & 100.00000 & 100.00000 & 100.00000 & 100.00000 \\
\hline 1 & 34.25550 & 49.65250 & 32.92550 & 36.96125 \\
\hline 2 & 24.09050 & 23.90775 & 27.71600 & 34.43300 \\
\hline 3 & 21.43850 & 20.36300 & 24.58850 & 32.85200 \\
\hline 4 & 20.84225 & 19.33750 & 22.72950 & 31.91725 \\
\hline 5 & 20.66725 & 18.90950 & 21.67150 & 31.41850 \\
\hline 6 & 20.57775 & 18.67575 & 21.08975 & 31.13300 \\
\hline 7 & 20.48750 & 18.49650 & 20.76725 & 30.97550 \\
\hline 8 & 20.35225 & 18.26925 & 20.57025 & 30.86700 \\
\hline 9 & 20.09375 & 17.86750 & 20.41300 & 30.75500 \\
\hline 10 & 19.58650 & 17.11825 & 20.22425 & 30.58350 \\
\hline 11 & 18.64175 & 15.69600 & 19.90550 & 30.23900 \\
\hline 12 & 16.89850 & 12.99275 & 19.26050 & 29.56425 \\
\hline 13 & 14.11125 & 8.68975 & 17.93850 & 28.17675 \\
\hline 14 & 10.45925 & 3.82150 & 15.03250 & 25.17275 \\
\hline
\end{tabular}

Table 4: Distribution of Energy Usage at node density 12 when $N=400$ and sch_len=15

\begin{tabular}{|c|c|c|c|c|}
\hline Active Time Units & STIC & MST_Edmonds & CSCA & SDT \\
\hline \hline 0 & 100.00000 & 100.00000 & 100.00000 & 100.00000 \\
\hline 1 & 32.11250 & 45.54600 & 29.22275 & 33.57350 \\
\hline 2 & 21.30500 & 20.15525 & 24.64050 & 31.41275 \\
\hline 3 & 18.64075 & 17.27875 & 21.87325 & 30.06450 \\
\hline 4 & 18.07650 & 16.45000 & 20.13850 & 29.25350 \\
\hline 5 & 17.92975 & 16.11175 & 19.12450 & 28.79700 \\
\hline 6 & 17.86925 & 15.92300 & 18.56825 & 28.57275 \\
\hline 7 & 17.77875 & 15.76350 & 18.25325 & 28.43300 \\
\hline 8 & 17.63875 & 15.54050 & 18.06525 & 28.33825 \\
\hline 9 & 17.35225 & 15.13675 & 17.92775 & 28.23750 \\
\hline 10 & 16.83075 & 14.37850 & 17.77125 & 28.08600 \\
\hline 11 & 15.87100 & 12.95975 & 17.49675 & 27.81300 \\
\hline 12 & 14.07800 & 10.26050 & 16.96275 & 27.19850 \\
\hline 13 & 11.34825 & 6.18925 & 15.81425 & 25.96175 \\
\hline 14 & 8.02375 & 2.19675 & 13.27450 & 23.28275 \\
\hline
\end{tabular}


Table 5: Percentage of Nodes with active time units 14 with respect to MST_Edmonds when $N=400$ and sch_len $=15$

\begin{tabular}{|c|c|c|c|c|}
\hline Node Density & STIC & MST_Edmonds & CSCA & SDT \\
\hline \hline 8 & 2.11 & 1.00 & 2.67 & 4.14 \\
\hline 10 & 2.74 & 1.00 & 3.93 & 6.59 \\
\hline 12 & 3.65 & 1.00 & 6.04 & 10.60 \\
\hline
\end{tabular}

Table 6: Percentage of Nodes with active time units 7 with respect to MST_Edmonds when $N=400$ and sch_len $=15$

\begin{tabular}{|c|c|c|c|c|}
\hline Node Density & STIC & MST_Edmonds & CSCA & SDT \\
\hline \hline 8 & 1.09 & 1.00 & 1.08 & 1.51 \\
\hline 10 & 1.11 & 1.00 & 1.12 & 1.67 \\
\hline 12 & 1.13 & 1.00 & 1.16 & 1.8 \\
\hline
\end{tabular}

Table 6. It is observed that MST_Edmonds makes considerable improvement with respect to other algorithms in reducing percentage of nodes with highest possible active time units and this improvement increases with the higher values of node density. Similarly, it also makes moderate improvements in reducing the percentage of nodes with active time units of 7 . Furthermore, these improvements are greater at higher node densities.

\subsubsection{Number of Node Transmissions}

In this section, we study the number of node transmissions needed by our heuristic algorithms as well as by the existing algorithms CSCA and SDT for the broadcast operation. In our experiments, the schedule length did not significantly affect the results for a given node density and thus we describe our results for sch_len=20. CSCA generates the minimum number of messages and this is expected as CSCA was designed to minimize the number of node transmissions. Our proposed algorithms are second next to CSCA as they minimize the number of node transmissions significantly with MST_Edmonds producing the best result. Although the results generated by STIC and MST_Edmonds are similar for lower values of $N$, the difference becomes more noticeable for larger values of $N$. As depicted in Figure 20, at node density 12 for $N=400$, MST_Edmonds, STIC and SDT generate number of node transmissions that is about $25 \%, 34 \%$ and $57 \%$ more than that of CSCA, respectively. A similar pattern of performance is observed for node density 8 and 


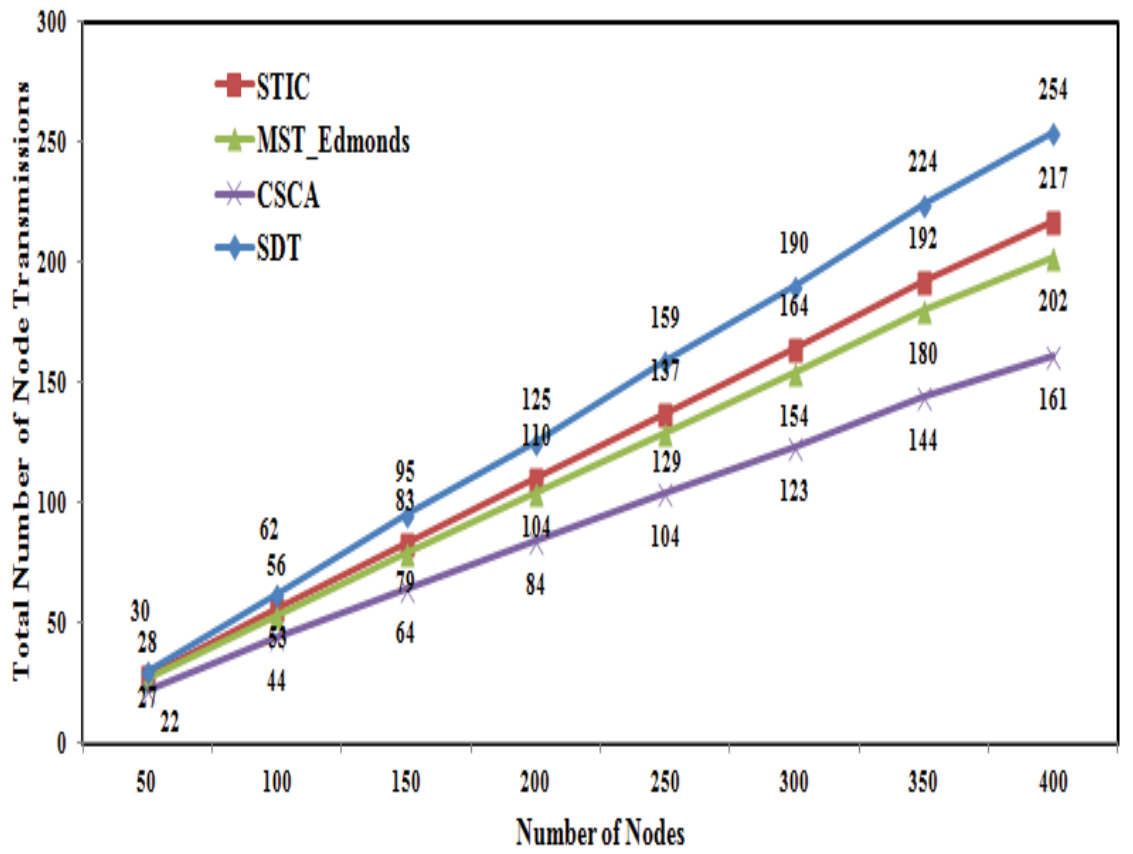

Figure 20: Total number of node transmissions at node density 12 for sch_len $=20$

10 as depicted in Figure 21. The number of node transmissions with respect to CSCA for all algorithms are shown in Table 7 for all node densities when $N=400$ and sch_len=20. It is observed that all the algorithms produce more node transmissions compared to CSCA, and this effect increases with higher values of node density while SDT generates the worst performance.

Table 7: Total Number of Node Transmissions with respect to CSCA when $N=400$, sch_len $=20$

\begin{tabular}{|c|c|c|c|c|}
\hline Node Density & STIC & MST_Edmonds & CSCA & SDT \\
\hline \hline 8 & 1.26 & 1.21 & 1.00 & 1.39 \\
\hline 10 & 1.29 & 1.23 & 1.00 & 1.48 \\
\hline 12 & 1.34 & 1.25 & 1.00 & 1.57 \\
\hline
\end{tabular}




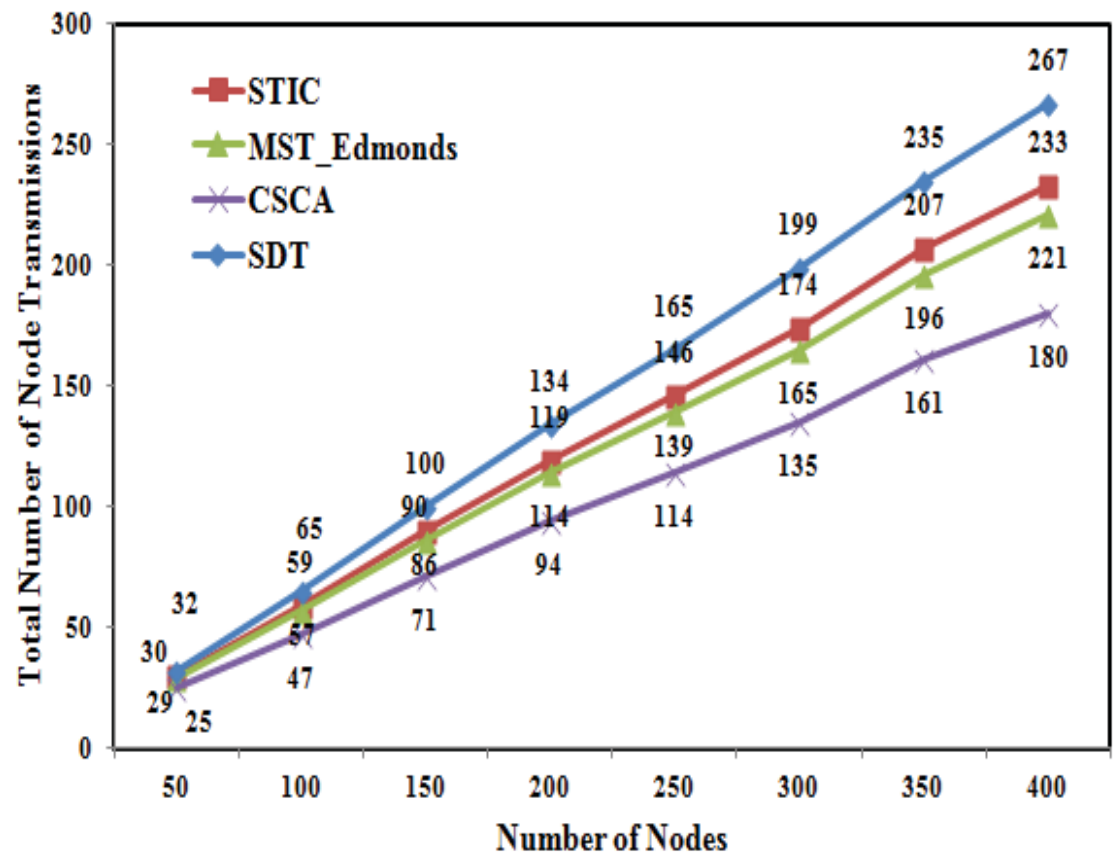

(a) node density 10

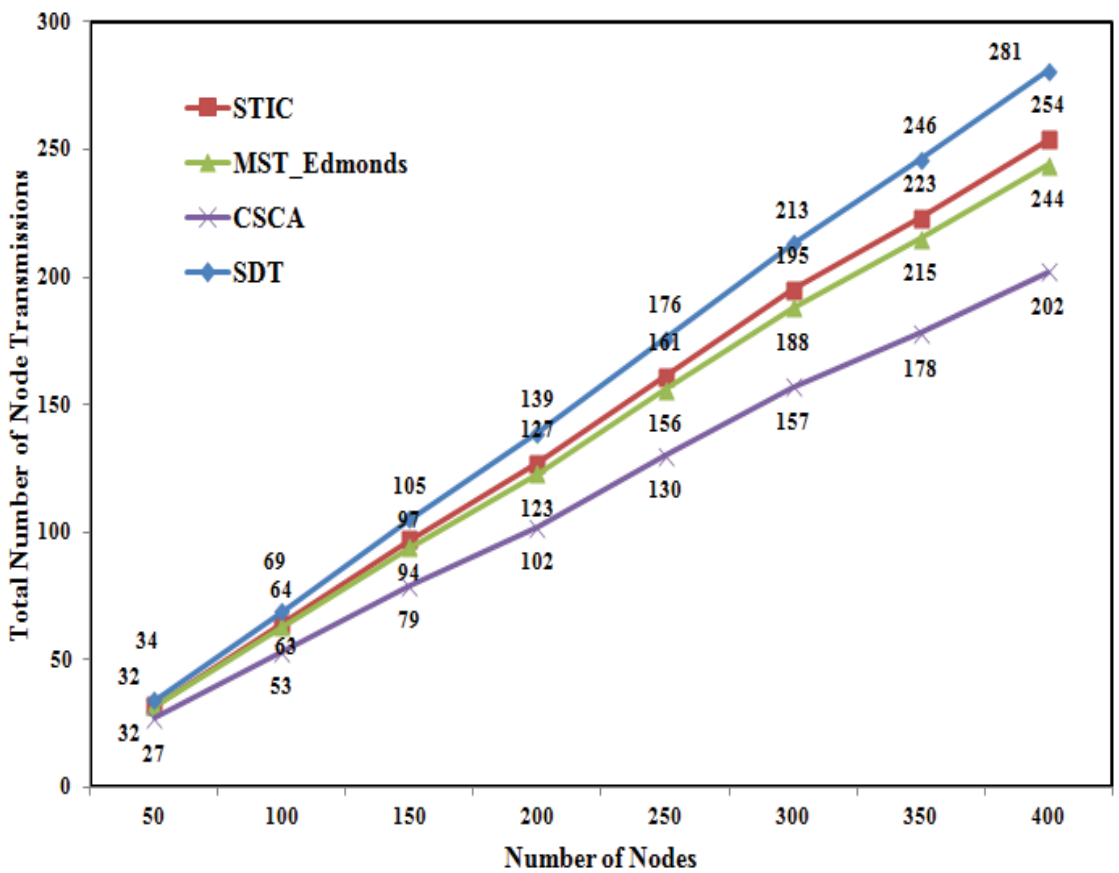

(b) node density 8

Figure 21: Total number of node transmissions for sch_len $=20$ 


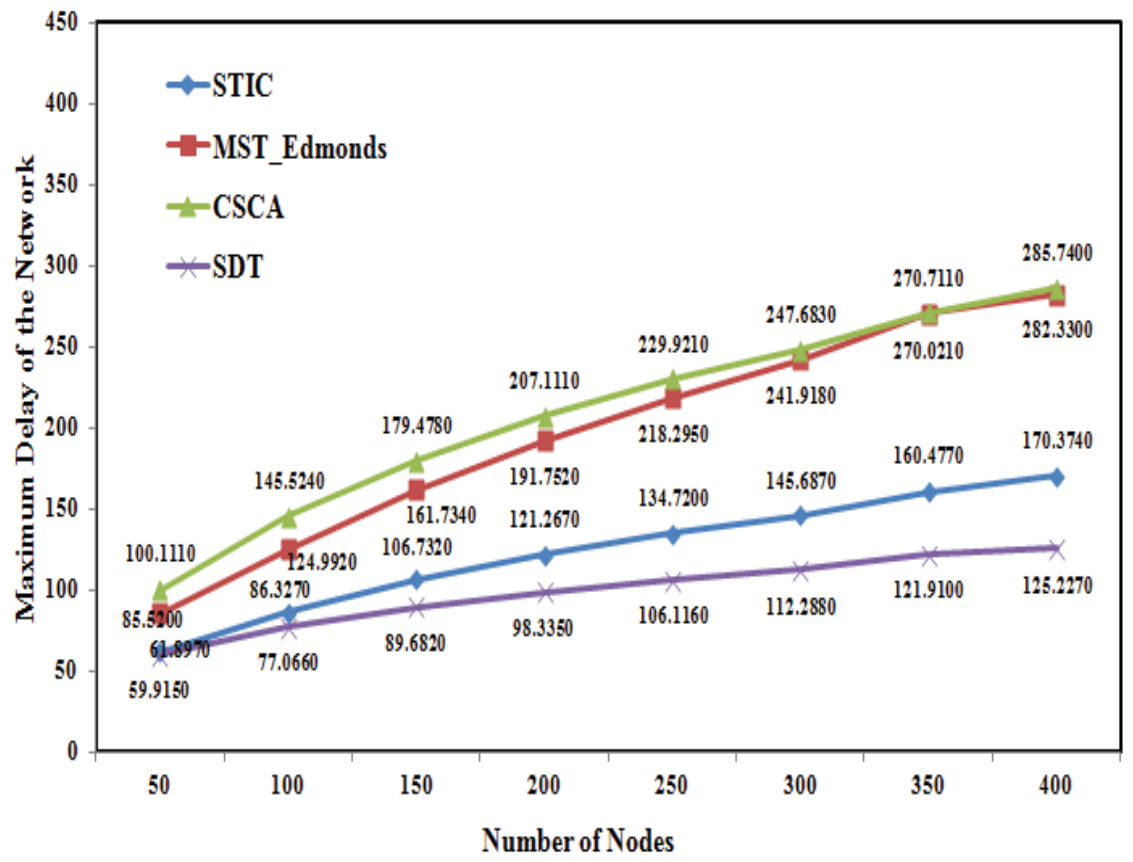

Figure 22: Maximum Delay of the Network at node density 12 for sch_len $=20$

\subsubsection{Maximum Delay of Broadcast Operation}

We describe our results for maximum delay with respect to sch_len 20. At all node densities, SDT generates the smallest value of maximum delay among all the algorithms. The STIC algorithm exhibits the closest performance to it. As depicted in Figure 22, at node density 12 for $N=400$, STIC produces maximum delay which is about $36.05 \%$ larger than that of SDT whereas MST_Edmonds and CSCA generate delay which is about $125.45 \%$ and $128 \%$ larger than that of the same algorithm, respectively. MST Edmonds performs in a similar way to CSCA at node density 12 for higher values of $N$ as shown in Figure 22 but for lower densities, it has worse performance than CSCA for larger values of N. The results for node density 10 and 8 are shown in Figures 23(a) and 23(b), respectively. For all node densities, the maximum delay generated by all the algorithms with respect to SDT are shown in Table 8. It is observed that maximum delay reduces slightly for STIC with higher values of node density while for CSCA, it gradually increases with increasing node densities compared to SDT. 


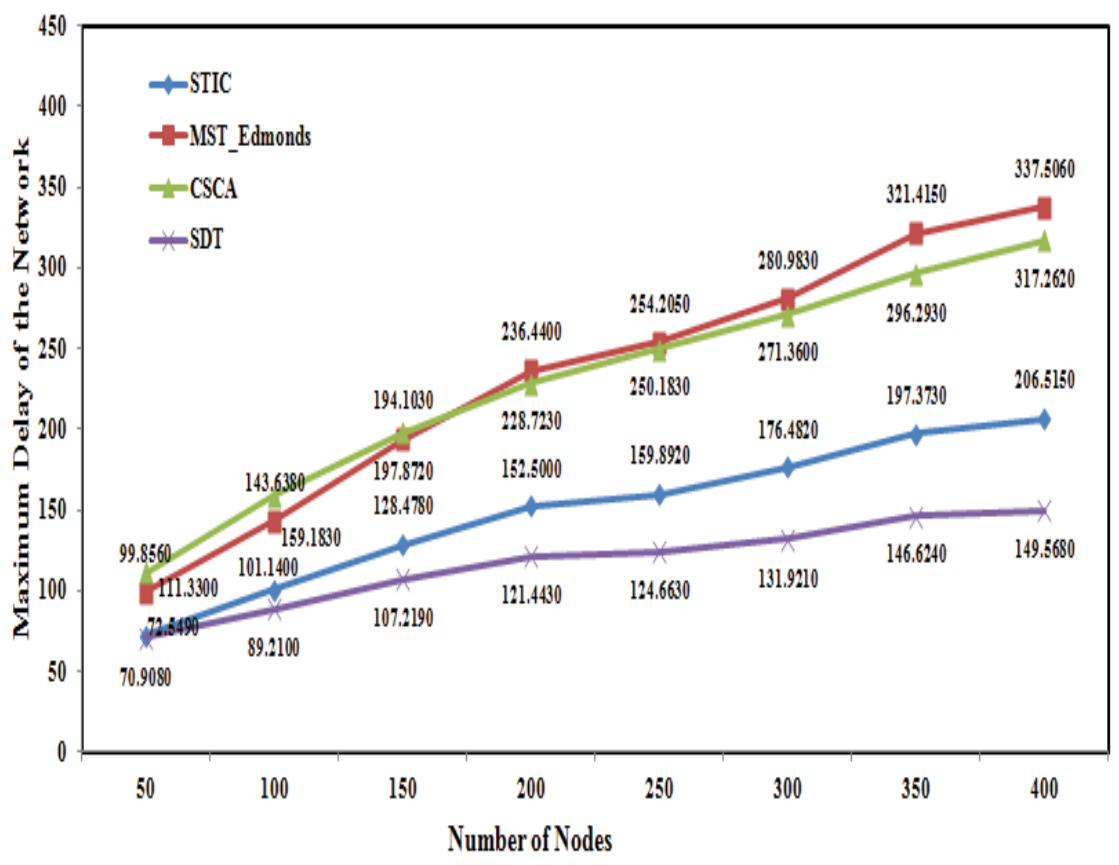

(a) node density 10

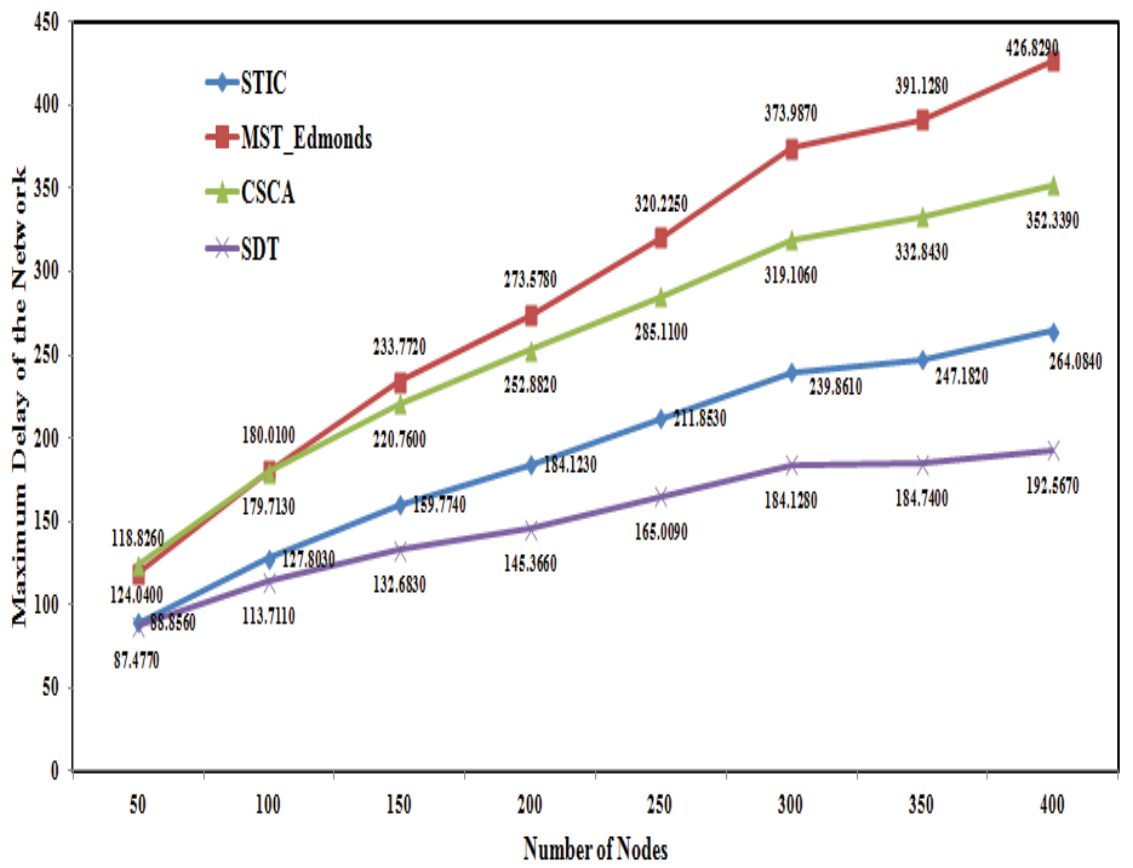

(b) node density 8

Figure 23: Maximum Delay of the Network at node density 10 and 8 for sch_len $=20$ 
Table 8: Normalized Maximum Delay with respect to SDT when $N=400$ and sch_len=20

\begin{tabular}{|c|c|c|c|c|}
\hline Node Density & STIC & MST_Edmonds & CSCA & SDT \\
\hline \hline 8 & 1.37 & 2.22 & 1.83 & 1.00 \\
\hline 10 & 1.38 & 2.26 & 2.12 & 1.00 \\
\hline 12 & 1.36 & 2.25 & 2.28 & 1.00 \\
\hline
\end{tabular}

\subsubsection{Average Delay of Broadcast Operation}

SDT generates the minimum average delay for broadcast operation at all node densities. Figures 24, 25(a) and 25(b), illustrate the result for node density 12, 10 and 8, respectively. STIC always generates the result closest to SDT and particularly at $N=50$, it produces lower delay than the SDT at all node densities. As depicted in Figure 24, at node density 12 for $N=400$, it produces about $22 \%$ more delay as compared to that of the SDT algorithm while MST_Edmonds generates about $116 \%$ more delay than the same algorithm. CSCA generates about $126 \%$ more delay than the SDT algorithm at the same node density for $N=400$. MST_Edmonds and CSCA generate about $77 \%$ and $85 \%$ more delay respectively compared to STIC at node density 12. MST_Edmonds exhibits the worst performance at node density 8 although it produces better result than CSCA upto $N=150$. Its performance improves with increasing node densities as and finally at node density 12, MST_Edmonds generates better result than CSCA for all values of $N$.

Table 9: Normalized Average Delay with respect to SDT when $N=400$ and sch_len=20

\begin{tabular}{|c|c|c|c|c|}
\hline Node Density & STIC & MST_Edmonds & CSCA & SDT \\
\hline \hline 8 & 1.28 & 2.15 & 1.81 & 1.00 \\
\hline 10 & 1.26 & 2.16 & 2.09 & 1.00 \\
\hline 12 & 1.22 & 2.16 & 2.26 & 1.00 \\
\hline
\end{tabular}

For all node densities, the average delay of all the algorithms with respect to SDT is shown in Table 9 for $N=400$ and sch_len=20. It is observed that with higher values of node density the performance of STIC improves while the performance of CSCA degrades compared to the SDT algorithm. 


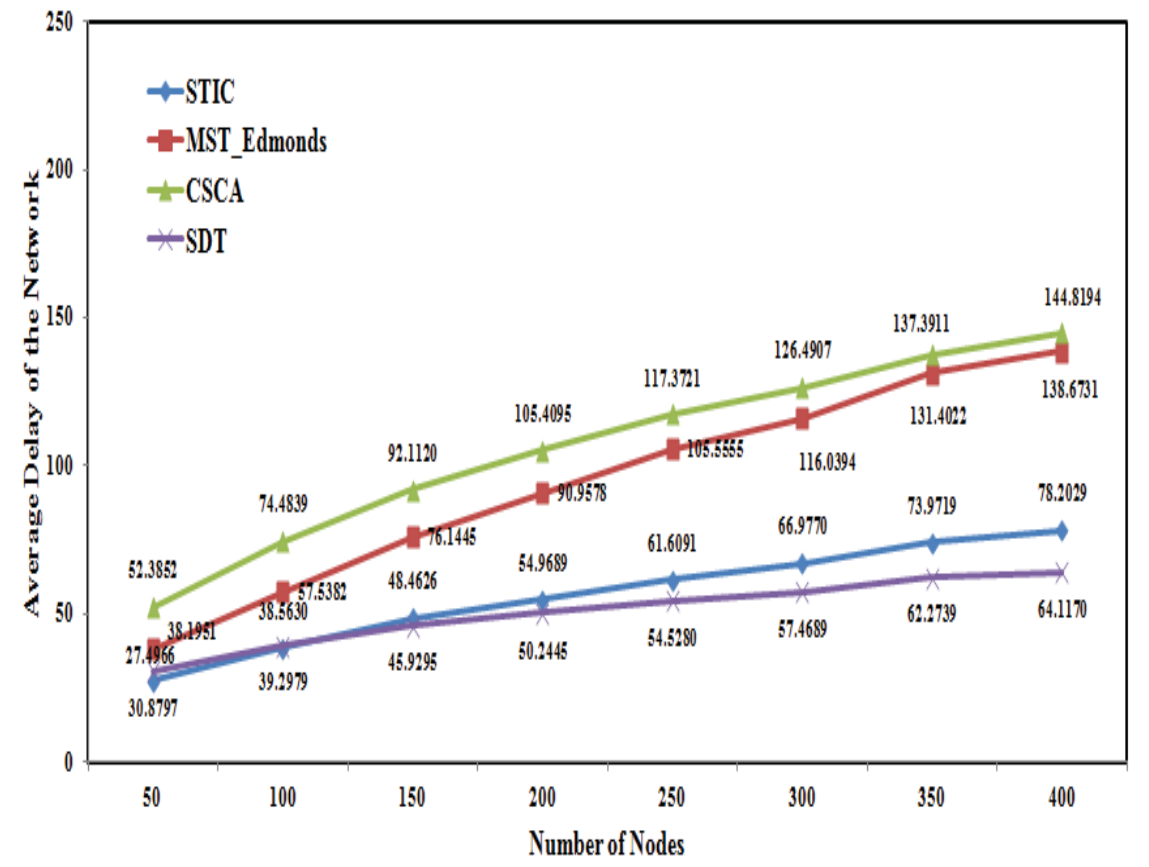

Figure 24: Average Delay of the Broadcast Opeation at node density 12 for sch_len $=20$ 


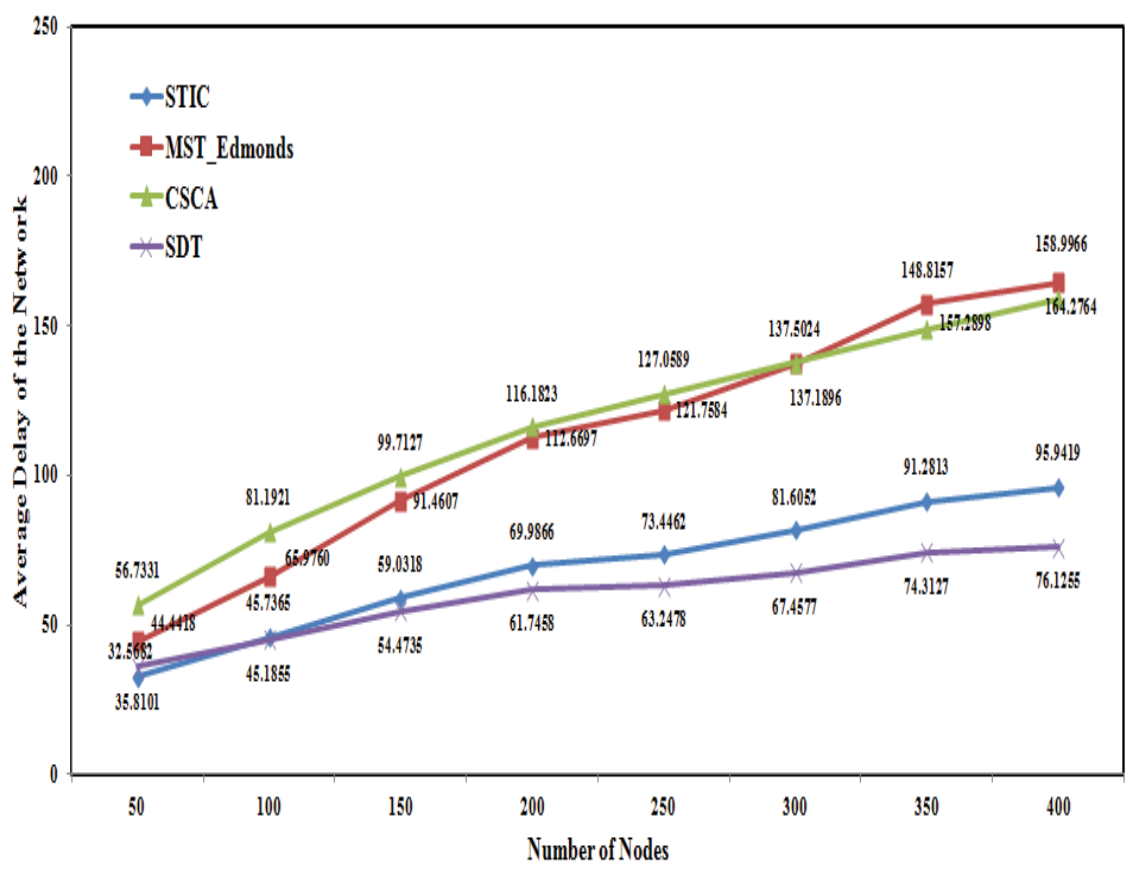

(a) node density 10

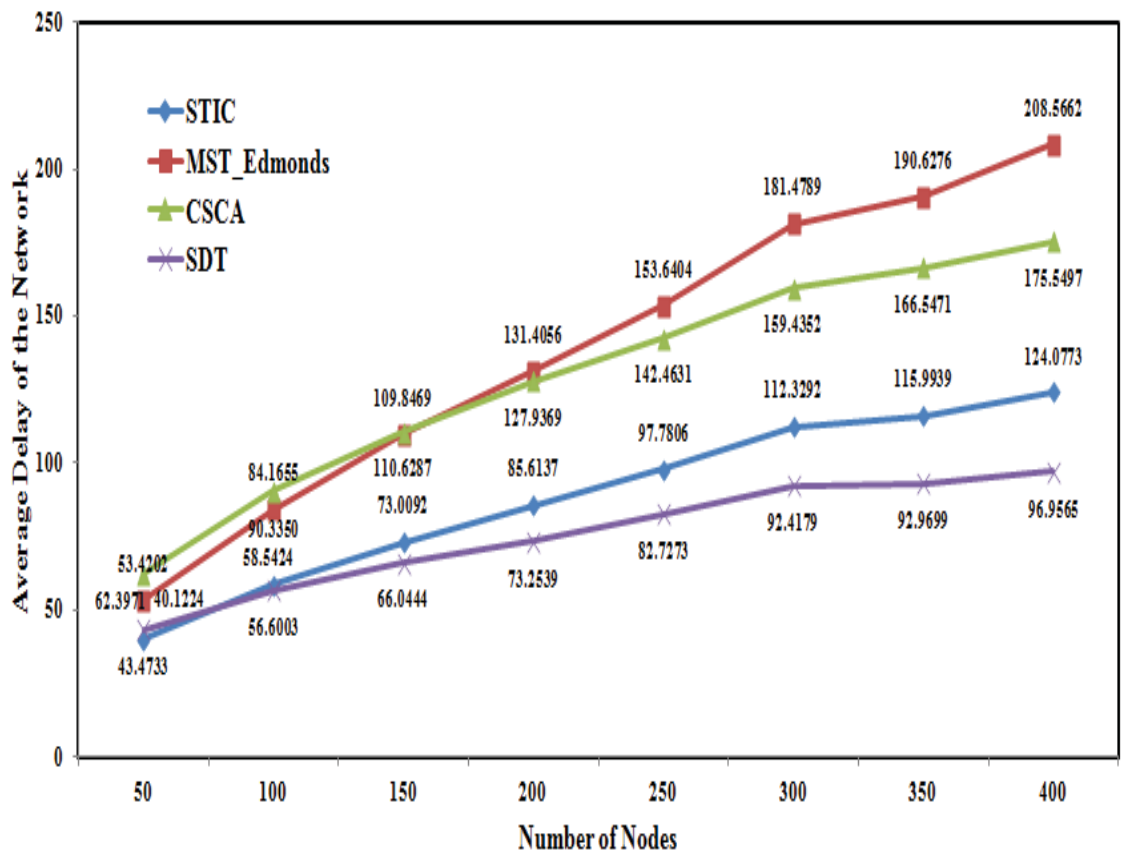

(b) node density 8

Figure 25: Average Delay of the Network at node density 10 and 8 for sch_len $=20$ 


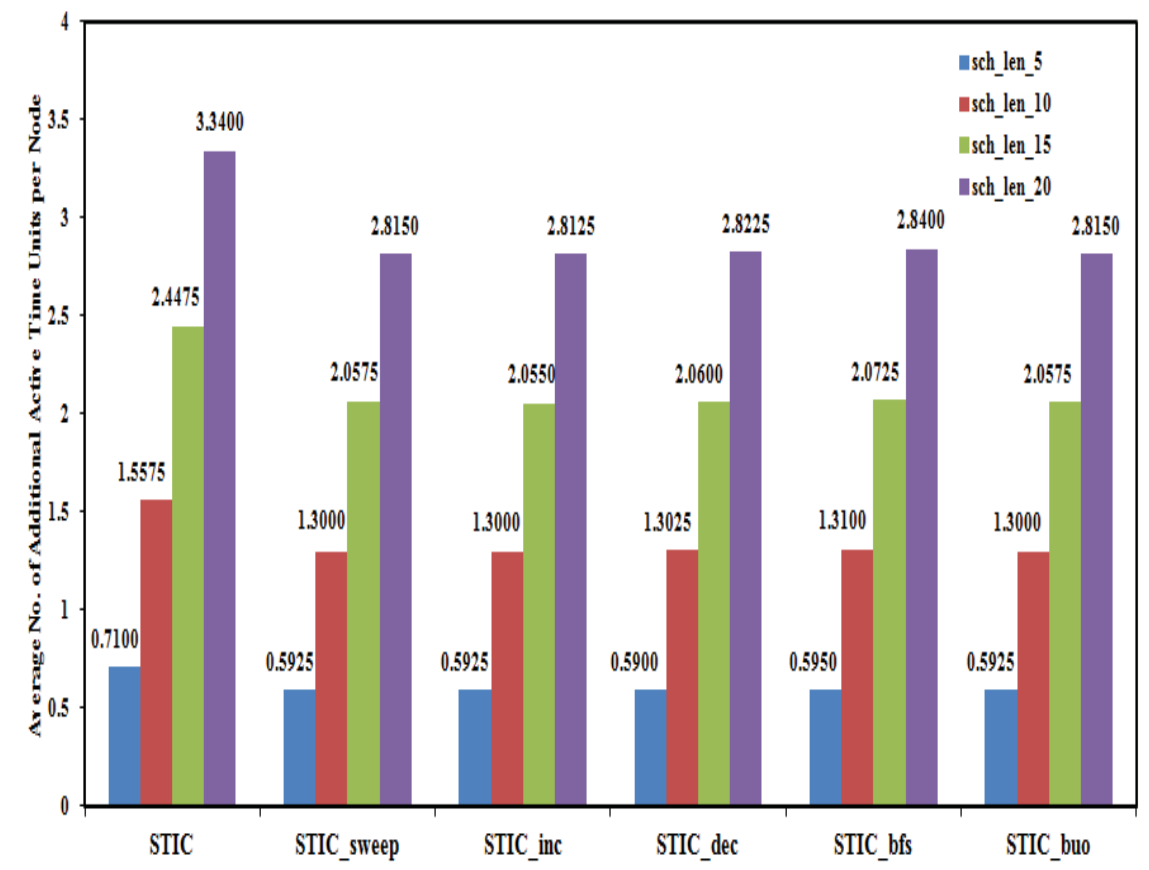

Figure 26: Average no. of additional active time units per node for various versions of STIC algorithm at node density $=12$ and $N=400$

\subsection{Performance Analysis of Various Versions of STIC Algo- rithm}

We apply sweep operations on the broadcast tree generated by the STIC algorithm in order to reduce the cost of the tree. Different variants of sweep operation differ in the order in which nodes of the tree are scanned. In this section we compare the performance of STIC with that produced by applying different type of sweep operations on the same algorithm.

\subsubsection{Average Number of Additional Active Time Units per Node}

Although different versions of sweep operation produce similar results, STIC_inc generates the best result among them. We describe our results for node density 12 and $N=400$. As shown in Figure 26, at sch_len=20 STIC produces average additional active time units which is about $18.75 \%$ more than that of STIC_inc. 


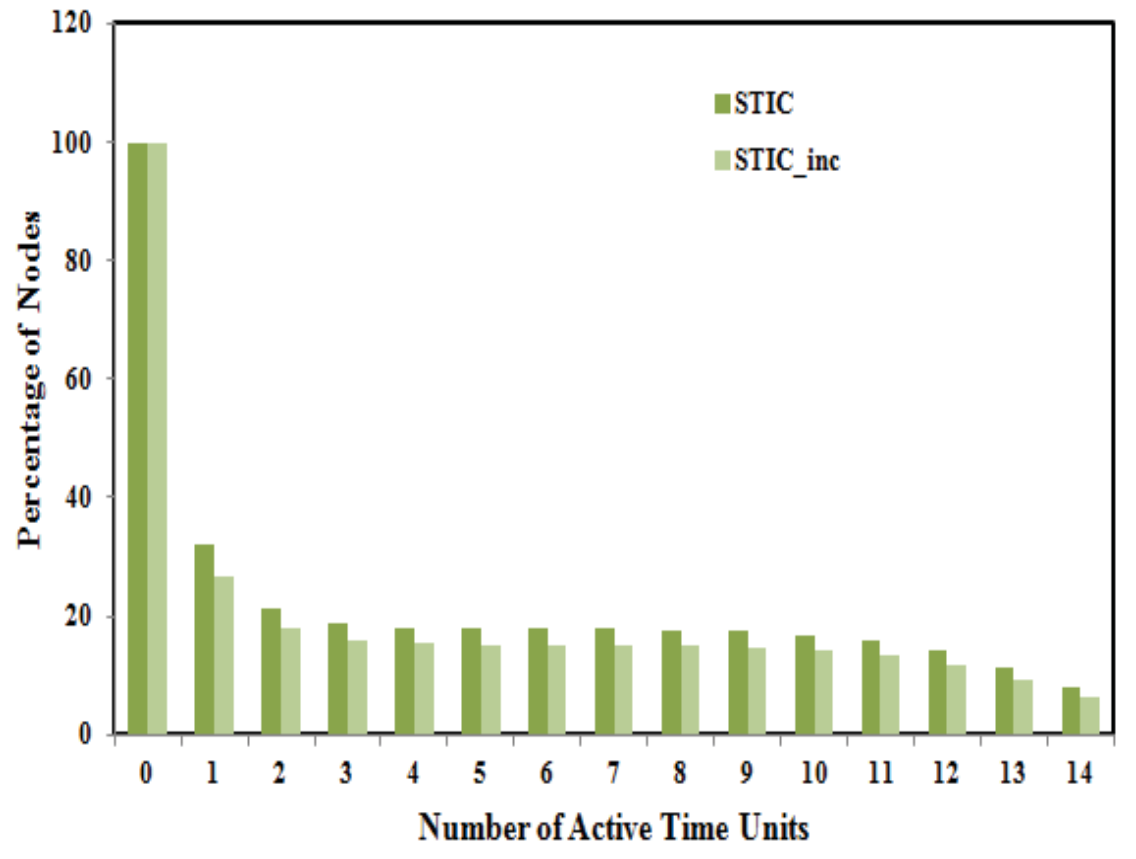

Figure 27: Distribution of Energy Usage for STIC and STIC_inc algorithms at node density $=12$ for $N=400$ and sch_len=15

\subsubsection{Energy Distribution}

We compare the energy usage of STIC with STIC_inc as it gives the best result among all variants of sweep operation. We describe the result for node density $12, N=400$ and sch_len=15. As shown in Figure 27, at node density 12, about 8.02\% nodes have active time units of 14 for STIC while about $6.343 \%$ of nodes have active time units of 14 for STIC_inc. Thus, given a schedule length STIC_inc is more capable of reducing the number of nodes with highest possible active time units. At node density 12, STIC generates about 1.26 times more nodes having active time units of 14 while it produces about 1.18 times more nodes with active time units of 7 compared to STIC_inc.

\subsubsection{Number of Node Transmissions}

STIC_inc gives the best result in terms of number of node transmissions and we analyze the result of STIC and STIC_inc for sch_len=20 at node density 12. As shown in Figure 28, at node density=12 for $N=400$, STIC generates about $17.30 \%$ more node transmissions as 


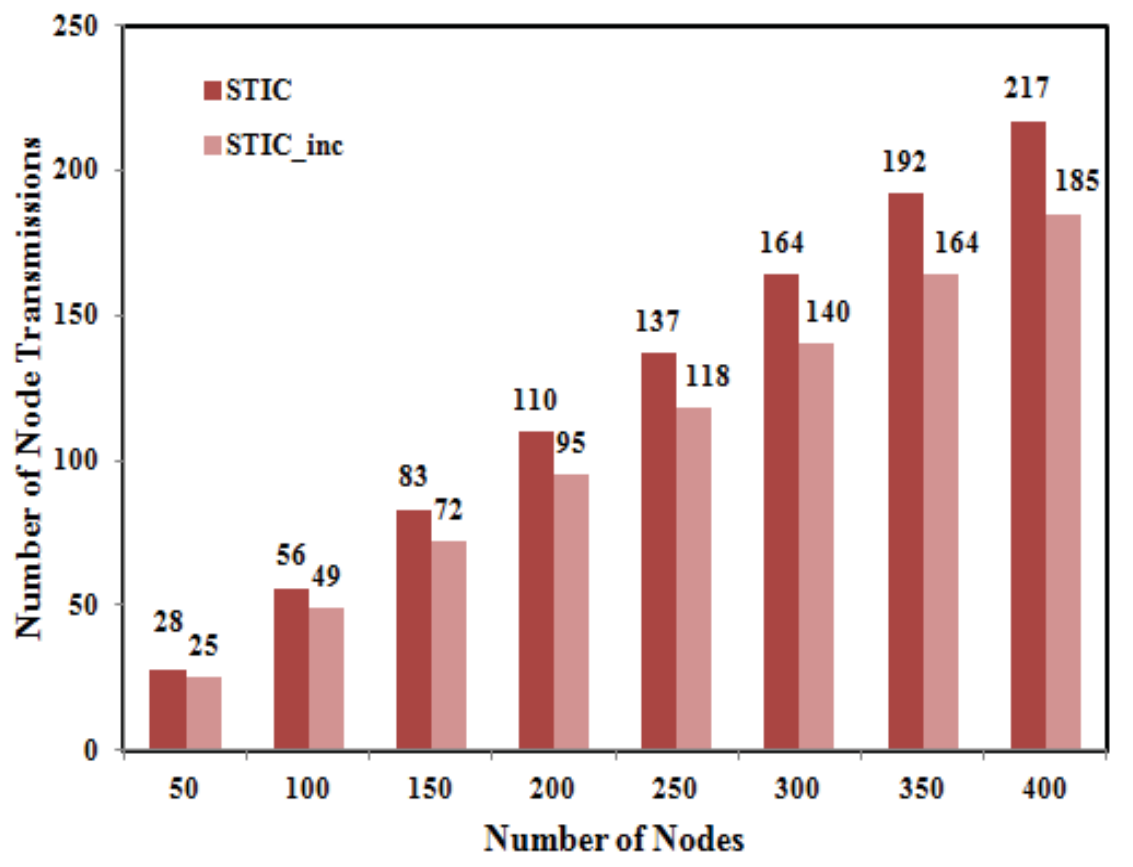

Figure 28: Number of node transmissions for STIC and STIC_inc at node density $=12$ for sch_len $=20$

compared to STIC_inc.

\subsubsection{Maximum Delay of Broadcast Operation}

The maximum delay of the broadcast operation increases if we apply sweep operations on the broadcast tree generated by the STIC algorithm. As a sweep operation changes the structure of broadcast trees, it may increase the delay as the parents of some nodes are changed. Among all versions of sweep operation, STIC_bfs produces the best result. We compare the maximum delay of STIC and STIC_bfs at node density 12 for sch_len 20. As shown in Figure 29, the maximum delay produced by STIC_bfs is slightly higher compared to STIC for lower values of $N$ but the differences become more noticeable at higher values of $N$. As illustrated in Figure 29, for $N=400$, the maximum delay generated by STIC is 170.37 whereas the maximum delay produced by STIC_bfs is 176.04 . 


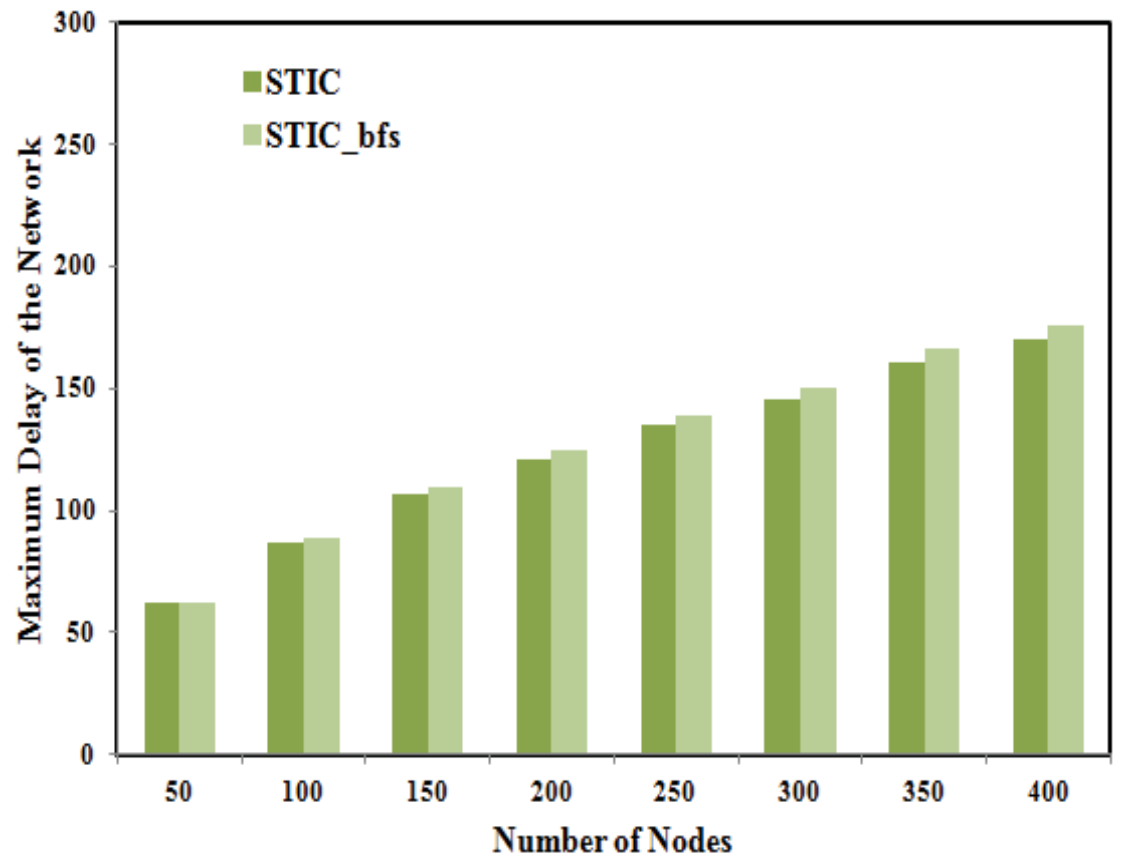

Figure 29: Maximum Delay of the Network for STIC and STIC_bfs at node density 12 for sch_len $=20$

\subsubsection{Average Delay of Broadcast Operation}

The average delay of the broadcast operation also can increase with the application of sweep operations for the same reason mentioned in Section 4.2.4. We compare the average delay of STIC_bfs with that of STIC as STIC_bfs exhibits the best result among all versions of sweep operation. As depicted in Figure 30, the average delay produced by STIC_bfs is slightly higher than that of STIC for lower values of $N$ while the difference increases with higher values of $N$.

\subsection{Performance Analysis of Various Versions of MST_Edmonds Algorithm}

In this section we compare the performance of MST_Edmonds with that produced by applying different types of sweep operation on the broadcast tree generated by MST_Edmonds. 


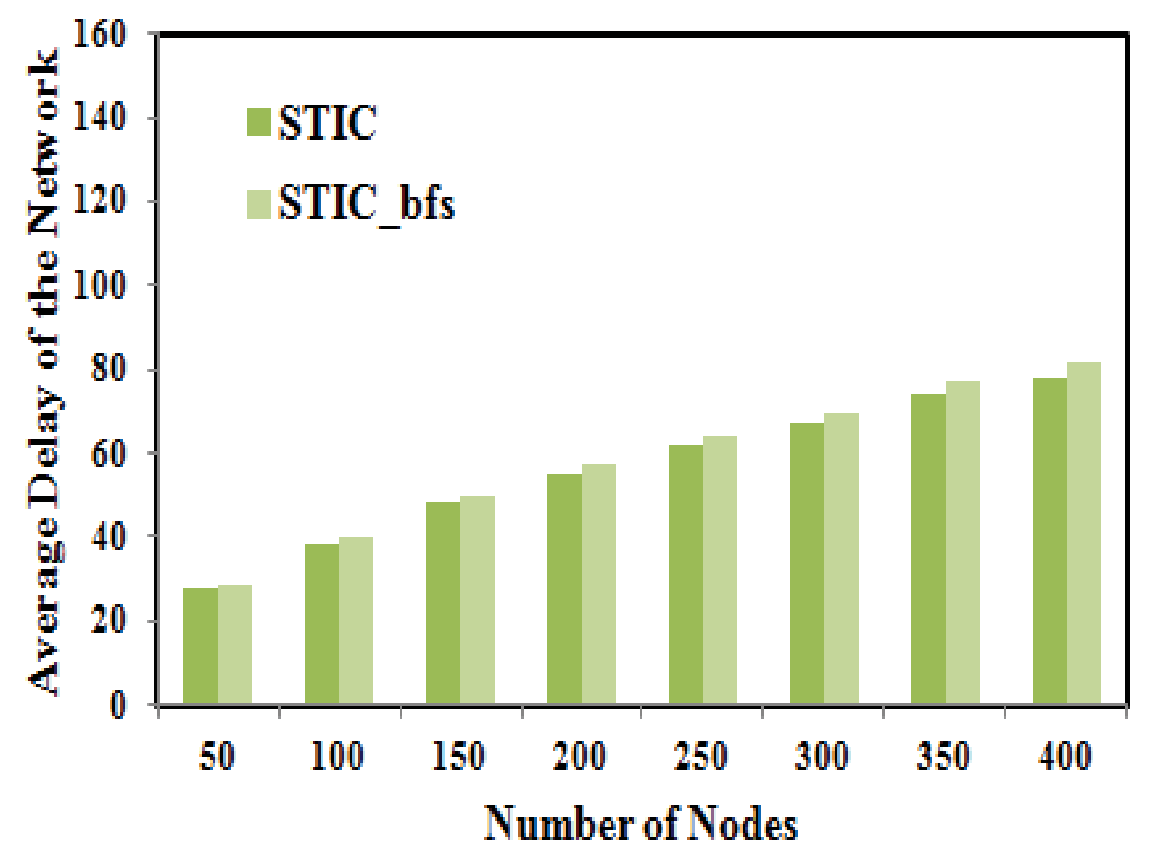

Figure 30: Average Delay of the Network for STIC and STIC_bfs at node density 12 for sch_len $=20$

\subsubsection{Average Number of Additional Active Time Units per Node}

MST_Edmonds_bfs produces the best result among various versions of the sweep operation. As illustrated in Figure 31, at node density=12 for $N=400$ and sch_len=20, MST_Edmonds produces about $16.86 \%$ more average additional active time units per node compared to MST_Edmonds_bfs.

\subsubsection{Energy Distribution}

We compare the energy usage of MST_Edmonds with MST_Edmonds_bfs as it gives the best result among all sweep variants. As shown in Figure 32, at node density 12 for $N=400$ and sch_len $=15$, about $2.20 \%$ and $1.64 \%$ of nodes have active time units of 14 for MST_Edmonds and MST_Edmonds_bfs, respectively. About $13.67 \%$ of nodes have active time units of 7 for MST_Edmonds_bfs while $15.76 \%$ nodes have active time units of 7 for MST_Edmonds. Thus at node density 12, MST_Edmonds generates about 1.34 times more nodes with active time units of 14 while it produces about 1.15 times more nodes with active time units of 7 


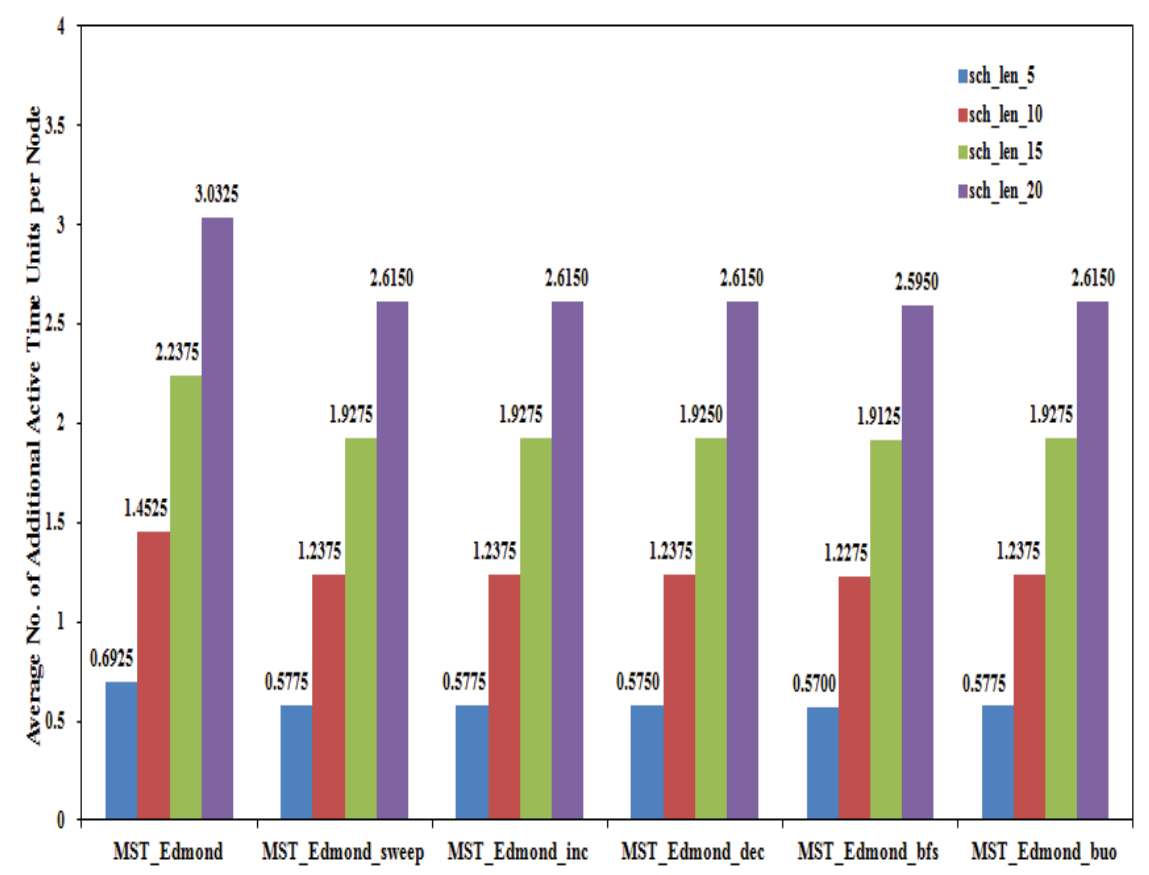

Figure 31: Average no. of additional active time units per node for various versions of MST_Edmonds algorithm at node density $=12$ and $N=400$

compared to MST_Edmonds_bfs.

\subsubsection{Number of Node Transmissions}

MST_Edmonds_bfs gives the best result in minimizing the number of node transmissions. We describe the result of MST_Edmonds and MST_Edmonds_bfs for sch_len=20 at node density 12. As shown in Figure 33 for $N=400$, MST_Edmonds generates about $17.44 \%$ more node transmissions compared to MST_Edmonds_bfs.

\subsubsection{Maximum Delay of Broadcast Operation}

Sweep operations on the broadcast tree generated by the MST_Edmonds algorithm decrease the maximum delay. Sweep operations modify the structure of the broadcast tree by changing the parents of some nodes and this way they may reduce the maximum delay of the broadcast tree. We compare the maximum delay of MSTEdmonds and MST_Edmonds_bfs at node density 12 for sch_len=20 as MST_Edmonds_bfs is best among all variants of sweep operation. As shown in Figure 34, at $N=400$, maximum delay of 




Figure 32: Distribution of Energy Usage for MST_Edmonds and MST_Edmonds_bfs algorithms at node density $=12$ for $N=400$ and sch_len $=15$

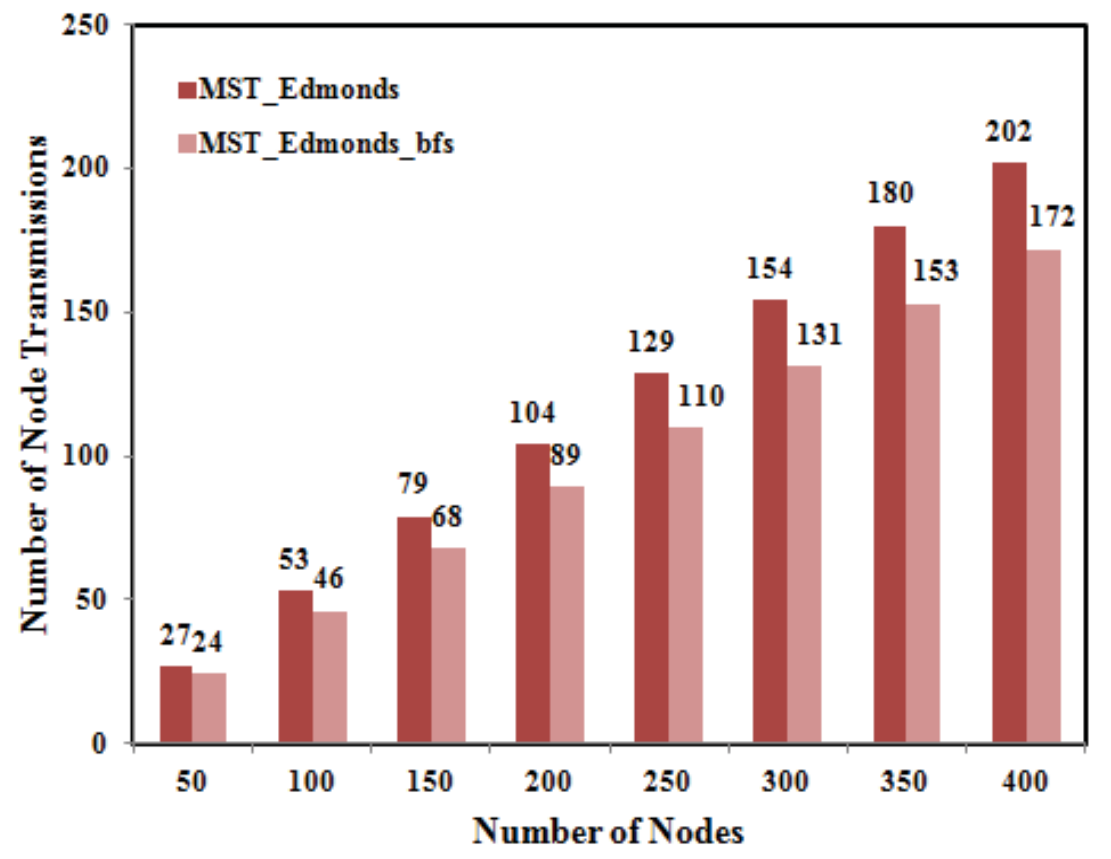

Figure 33: Number of node transmissions for MST_Edmonds and MST_Edmonds_bfs at node density $=12$ for sch_len $=20$ 


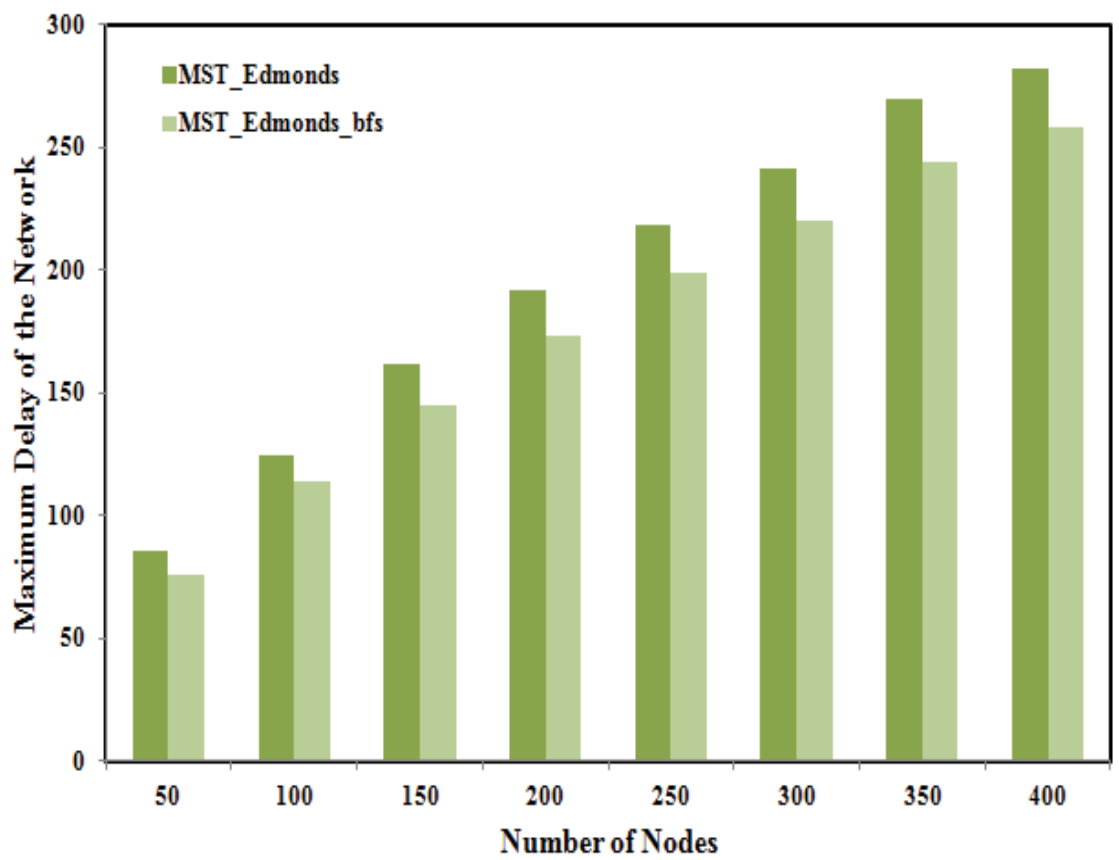

Figure 34: Maximum Delay of the Network for MST_Edmonds and MST_Edmonds_bfs at node density 12 for sch_len $=20$

MST_Edmonds_bfs is 258.742 while the maximum delay produced by MST_Edmonds is 282.33. Thus MST_Edmonds generates about 9.11\% more delay compared to MST_Edmonds_bfs for $N=400$.

\subsubsection{Average Delay of Broadcast Operation}

Sweep operations also reduce the average delay of the broadcast operation. MST_Edmonds_bfs generates the best result among all versions of sweep operation and thus we compare the average delay of MST_Edmonds and MST_Edmonds_bfs at node density 12 for sch_len=20. As depicted in Figure 35, MST_Edmonds produces about $8.17 \%$ more average delay as compared to MST_Edmonds_bfs at $N=400$. 


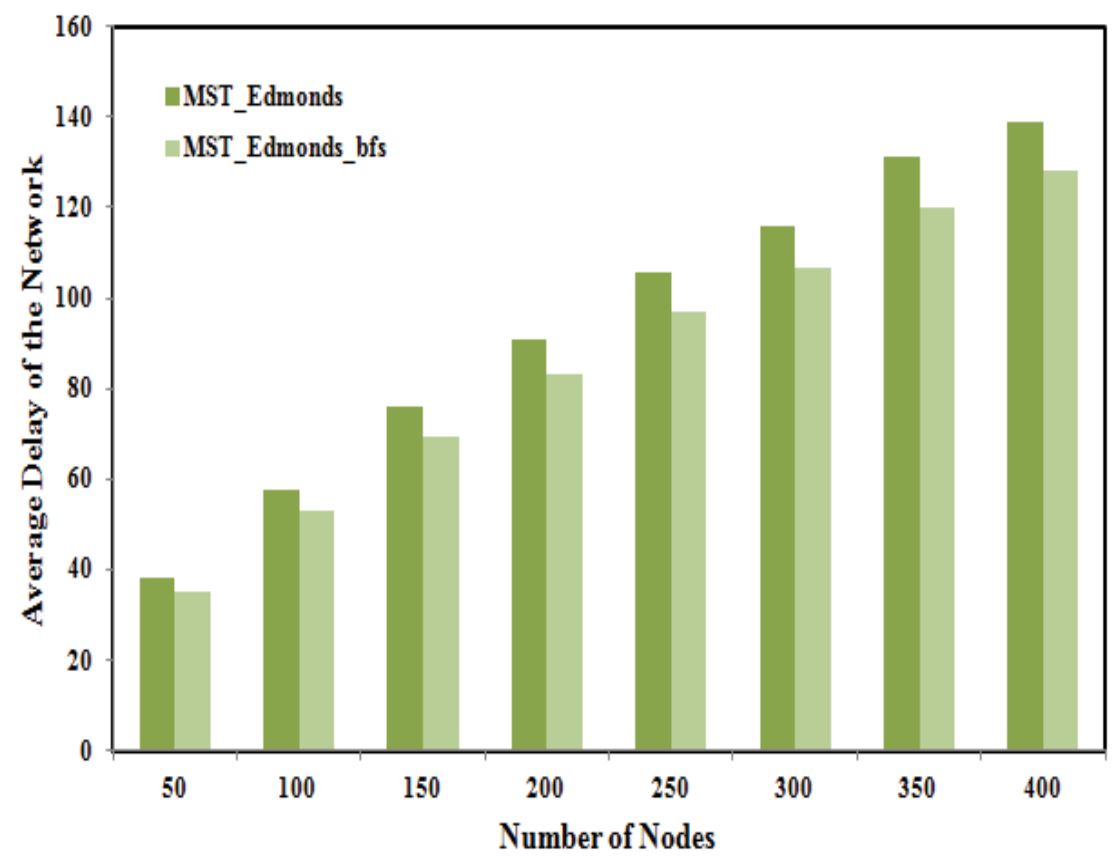

Figure 35: Average Delay of the Network for MST_Edmonds and MST_Edmonds_bfs at node density 12 for sch_len $=20$ 
Table 10: Improvements obtained by the sweep operation at node density 12 for $N=400$ : metric for best variant of sweep divided by metric for algorithm without sweep

\begin{tabular}{|c|c|c|c|c|}
\hline Cost Measure & STIC & MST_Edmonds & CSCA & SDT \\
\hline \hline $\begin{array}{c}\text { Additional active time units } \\
\text { per node for sch_len }=20\end{array}$ & 0.84 & 0.86 & 0.84 & 0.69 \\
\hline $\begin{array}{c}\text { Percentage of nodes with active } \\
\text { time units 14 for sch_len }=15\end{array}$ & 0.79 & 0.75 & 0.81 & 0.61 \\
\hline $\begin{array}{c}\text { Number of Node Transmissions } \\
\text { for } s c h \_l e n=20\end{array}$ & 0.85 & 0.85 & 0.93 & 0.83 \\
\hline Maximum Delay for $s c h \_l e n=20$ & 1.03 & 0.92 & 0.96 & 1.16 \\
\hline Average Delay for $s c h \_l e n=20$ & 1.04 & 0.92 & 0.96 & 1.11 \\
\hline
\end{tabular}

\subsection{Summary of Effect of Sweep Operation}

As shown in Table 10, all variants of sweep operation generally reduce all the cost measures for all our algorithms. The only exceptions are the maximum and average delay for STIC and SDT. However, the sweep variant that gives the best result for different combinations of cost measure and broadcast tree algorithm is not always the same. The best sweep variant for different combinations is presented in Table 11. In the remaining sections, we always use the best sweep variant of the algorithm when comparing with other algorithms.

Table 11: Best version of sweep operation for various combinations of broadcast tree algorithm and cost measure

\begin{tabular}{|c|c|c|c|c|}
\hline cost measure & MST_Edmonds & STIC & CSCA & SDT \\
\hline \hline $\begin{array}{c}\text { Additional active time } \\
\text { units per node }\end{array}$ & bfs & inc & bfs & inc \\
\hline Energy Usage of Nodes & bfs & inc & bfs & inc \\
\hline Number of Node Transmissions & bfs & inc & bfs & inc \\
\hline Maximum Delay & bfs & no sweep & bfs & no sweep \\
\hline Average Delay & bfs & no sweep & bfs & no sweep \\
\hline
\end{tabular}

\subsection{Performance Comparison of all Algorithms with Sweep Operations}

In this section, we compare the performance of STIC, MST_Edmonds, CSCA [18] and SDT [22] after applying the sweep operations on the broadcast tree generated by these 
Table 12: Average Additional Active Time Units per Node for $N=400$ and sch_len $=20$

\begin{tabular}{|c|c|c|c|c|}
\hline Node Density & STIC_inc & MST_Edmonds_bfs & CSCA_bfs & SDT_inc \\
\hline \hline 8 & 3.8800 & 3.6275 & 4.1150 & 4.6075 \\
\hline 10 & 3.2550 & 3.0150 & 3.4650 & 4.0850 \\
\hline 12 & 2.8125 & 2.5950 & 3.0025 & 3.7325 \\
\hline
\end{tabular}

Table 13: Average Additional Active Time Units per Node with respect to MST_Edmonds_bfs for $N=400$ and sch_len $=20$

\begin{tabular}{|c|c|c|c|c|}
\hline Node Density & STIC_inc & MST_Edmonds_bfs & CSCA_bfs & SDT_inc \\
\hline \hline 8 & 1.07 & 1.00 & 1.13 & 1.27 \\
\hline 10 & 1.08 & 1.00 & 1.15 & 1.35 \\
\hline 12 & 1.08 & 1.00 & 1.16 & 1.44 \\
\hline
\end{tabular}

algorithms.

\subsubsection{Average Number of Additional Active Time Units per Node}

For all node densities, we describe our results with respect to $N=400$ and sch_len $=20$. The results are given in Tables 12 and 13. Both STIC_inc and MST_Edmonds_bfs utilize a smaller number of additional active time units per node as compared to CSCA_bfs and SDT_inc, while MST_Edmonds_bfs produces slightly better result than the STIC_inc algorithm. For all node densities and schedule lengths, SDT_inc exhibits the worst performance among all the algorithms. With higher values of node density all algorithms utilize fewer additional active time units per node, which is to be expected.

As depicted in Table 12, at node density 12 for sch_len $=20$, the average additional active time units for MST_Edmonds_bfs and STIC_inc are 2.5950 and 2.8125, respectively. Thus, on average, a node has to stay awake for additional time which about $13 \%$ and $14 \%$ of the schedule to participate in the broadcast operation for MST_Edmonds_bfs and STIC_inc, respectively. CSCA_bfs causes a node to remain alive for about $15 \%$ of the schedule and SDT_inc causes a node to be active for about $19 \%$ of the schedule for the same value of sch_len. At node density 12, STIC_inc, CSCA_bfs and SDT_inc produce about 8.4\%, 16\% and $44 \%$ more additional active time units per node compared to MST_Edmonds_bfs. At the same node density, CSCA_bfs and SDT_inc generate about $6.76 \%$ and $32.7 \%$ more 
additional active time units per node compared to STIC_inc, respectively.

As depicted in Table 10, all the algorithms produce improved results after the application of sweep operation for average additional active time units per node. Although SDT_inc produces the worst result in all node densities, sweep operation makes significant improvement on the result produced by SDT. The relative improvement for SDT is higher than that for MST_Edmonds, thereby reducing the latter's performance advantage. As can be seen from Table 1, at node density 12, SDT produces about $78 \%$ more additional active time units per node compared to MST_Edmonds while SDT_inc generates about $44 \%$ more additional active time units compared to MST_Edmonds_bfs as depicted in Table 13. It is also observed from Table 13, MST_Edmonds_bfs produces better result compared to all other algorithms with increasing values of node density.

\subsubsection{Distribution of Energy Usage}

For all node densities, we show the percentage of nodes with active time units of 14 and 7 produced after application of the sweep operation for $N=400$ and sch_len=15 in Tables 14 and 15 , respectively.

Table 14: Percentage of Nodes with active time units 14 when $N=400$ and sch_len=15

\begin{tabular}{|c|c|c|c|c|}
\hline Node Density & STIC_inc & MST_Edmonds_bfs & CSCA_bfs & SDT_inc \\
\hline \hline 8 & 10.97250 & 4.90050 & 14.88450 & 17.31100 \\
\hline 10 & 8.24525 & 2.83375 & 12.43975 & 15.47150 \\
\hline 12 & 6.34300 & 1.63725 & 10.80100 & 14.30600 \\
\hline
\end{tabular}

Table 15: Percentage of Nodes with active time units 7 when $N=400$ and sch_len=15

\begin{tabular}{|c|c|c|c|c|}
\hline Node Density & STIC_inc & MST_Edmonds_bfs & CSCA_bfs & SDT_inc \\
\hline \hline 8 & 20.61025 & 18.83225 & 21.06150 & 24.17850 \\
\hline 10 & 17.30750 & 15.80750 & 17.70950 & 21.62450 \\
\hline 12 & 15.02175 & 13.66950 & 15.32575 & 19.74850 \\
\hline
\end{tabular}

As shown in Table 14, at node density 12 only $1.64 \%$ of nodes have active time units of 14 for MST_Edmonds_bfs, $6.34 \%$ of nodes have active time units of 14 for STIC_inc, while $10.80 \%$ and $14.31 \%$ nodes have active time units of 14 for CSCA_bfs and SDT_inc, 
respectively. Thus MST_Edmonds_bfs significantly reduces the number of nodes with highest possible active time units. STIC_inc generates about 3.87 times more nodes with active time units of 14 than the MST_Edmonds_bfs algorithm. CSCA_bfs and SDT_inc produce about 6.59 and 8.74 times more such nodes, respectively compared to MST_Edmonds_bfs.

Also as shown in Table 15, at node density 12, only $13.67 \%$ nodes have active time units of 7 for MST_Edmonds_bfs while 15.02\%, $15.33 \%$ and $19.75 \%$ nodes have active time units of 7 for STIC_inc, CSCA_bfs and SDT_inc, respectively. This in turn indicates that STIC_inc, CSCA_bfs and SDT_inc produce about 1.10, 1.12 and 1.44 times more such nodes, respectively compared to MST_Edmonds_bfs. Thus, MST_Edmonds_bfs not only constructs a tree with the lowest average number of additional active time units per node, it also appears to create fewer highly loaded nodes and thus increasing the network lifetime. For all node densities, the percentage of nodes with active time units 14 and 7 for all algorithms with respect to MST_Edmonds_bfs are shown in Tables 16 and 17, respectively.

Table 16: Percentage of Nodes with active time units of 14 with respect to MST_Edmonds_bfs when $N=400$ and sch_len $=15$

\begin{tabular}{|c|c|c|c|c|}
\hline Node Density & STIC_inc & MST_Edmonds_bfs & CSCA_bfs & SDT_inc \\
\hline \hline 8 & 2.24 & 1.00 & 3.04 & 3.53 \\
\hline 10 & 2.91 & 1.00 & 4.39 & 5.46 \\
\hline 12 & 3.87 & 1.00 & 6.60 & 8.74 \\
\hline
\end{tabular}

Table 17: Percentage of Nodes with active time units of 7 with respect to MST_Edmonds_bfs when $N=400$ and sch_len $=15$

\begin{tabular}{|c|c|c|c|c|}
\hline Node Density & STIC_inc & MST_Edmonds_bfs & CSCA_bfs & SDT_inc \\
\hline \hline 8 & 1.09 & 1.00 & 1.12 & 1.28 \\
\hline 10 & 1.09 & 1.00 & 1.12 & 1.37 \\
\hline 12 & 1.10 & 1.00 & 1.12 & 1.44 \\
\hline
\end{tabular}

As can be seen from Table 16 and 17, MST_Edmonds_bfs exhibits better performance with higher values of node density compared to all other algorithms. SDT_inc produces worst performance in all node densities but its performance gap with respect to MST_Edmonds_bfs reduces more compared to that of the SDT with respect to MST_Edmonds. As shown in Table 5, at node density 12, SDT has 10.60 times the number of nodes with 
14 active time units compared to MST_Edmonds, while SDT_inc has 8.74 times such nodes compared to MST_Edmonds_bfs. It is observed from Tables 10, the sweep operation significantly reduces the percentage of nodes with active time units 14 while it moderately reduces the percentage of nodes with active time units of 7 for all algorithms as shown in Table 6 and 17.

\subsubsection{Number of Node Transmissions}

We describe the performance of the algorithms with respect to $N=400$ and sch_len=20 for all node densities and the results are shown in Tables 18 and 19. At all node densities, CSCA_bfs demonstrates the best result while SDT_inc exhibits the worst performance. Our algorithms MST_Edmonds_bfs and STIC_inc are next to CSCA_bfs in reducing the number of total transmissions. MST_Edmond_bfs consistently generates the least number of total broadcast messages as compared to STIC_inc. The result produced by the STIC_inc and MST_Edmonds_bfs are very close for smaller values of $N$, the differences become wider for larger values of $N$. It is observed from Table 19 that the number of node transmissions for Table 18: Number of Node Transmissions when $N=400$ and sch_len=20

\begin{tabular}{|c|c|c|c|c|}
\hline Node Density & STIC_inc & MST_Edmonds_bfs & CSCA_bfs & SDT_inc \\
\hline \hline 8 & 223 & 211 & 192 & 237 \\
\hline 10 & 202 & 189 & 167 & 222 \\
\hline 12 & 185 & 172 & 149 & 210 \\
\hline
\end{tabular}

Table 19: Number of Node Transmissions with respect to CSCA_bfs when $N=400$ and sch_len $=20$

\begin{tabular}{|c|c|c|c|c|}
\hline Node Density & STIC_inc & MST_Edmonds_bfs & CSCA_bfs & SDT_inc \\
\hline \hline 8 & 1.16 & 1.10 & 1.00 & 1.23 \\
\hline 10 & 1.21 & 1.13 & 1.00 & 1.33 \\
\hline 12 & 1.24 & 1.15 & 1.00 & 1.41 \\
\hline
\end{tabular}

all algorithms increases with respect to CSCA_bfs with higher values of node density. The sweep operation considerably improves the performance of SDT. As shown in Table 7, SDT produces about $57 \%$ more node transmissions compared to CSCA while SDT_inc generates about $41 \%$ more node transmissions compared to CSCA_bfs for node density 12(as shown 
in Table 19).

\subsubsection{Maximum Delay of Broadcast Operation}

In contrast to the previous cost measures, all versions of the sweep operation increase the maximum delay for STIC and SDT, while they reduce the maximum delay for MST_Edmonds and CSCA as shown in Table 10. Our results for maximum delay are illustrated in Figures 36 and 37 with respect to sch_len 20. At all node densities, SDT produces the smallest value for maximum delay of the broadcast operation. Our algorithm STIC generates the performance nearest to it. As depicted in Figure 37(b), at node density 8 MST_Edmonds_bfs exhibits better performance than CSCA_bfs below $N=150$ and then the delay of MST_Edmonds_bfs gradually increases with respect to CSCA_bfs. At node density 10, MST_Edmonds_bfs is doing better than CSCA_bfs below $N=350$ while at node density 12, MST_Edmonds_bfs shows better result for all values of $N$ compared to CSCA_bfs. At node density 12 for $N=400$, MST_Edmonds_bfs generates $106.62 \%$, STIC produces about $36.05 \%$ and CSCA_bfs produces about $119.91 \%$ more delay than SDT. The maximum delay of all algorithms with respect to SDT are shown in Table 20. As depicted in Table 20, the Table 20: Normalized Maximum Delay with respect to SDT when $N=400$ and sch_len=20

\begin{tabular}{|c|c|c|c|c|}
\hline Node Density & STIC & MST_Edmonds_bfs & CSCA_bfs & SDT \\
\hline \hline 8 & 1.37 & 2.02 & 1.77 & 1.00 \\
\hline 10 & 1.38 & 2.06 & 2.05 & 1.00 \\
\hline 12 & 1.36 & 2.07 & 2.20 & 1.00 \\
\hline
\end{tabular}

performance of SDT improves compared to the other algorithms with higher values of node density. It is observed that the application of sweep operation improves the performance of the MST_Edmonds_bfs and CSCA_bfs algorithm with respect to SDT. As depicted in Table 8, at node density 12, MST_Edmonds produces about $125 \%$ more delay than SDT while MST_Edmonds_bfs generates about $106.62 \%$ more delay compared to SDT as shown in Table 20. Similary, CSCA generates about 128\%(as shown in Table 8) and CSCA_bfs produces $119.91 \%$ (as shown in Table 20) more delay compared to SDT for the same value of node density. 


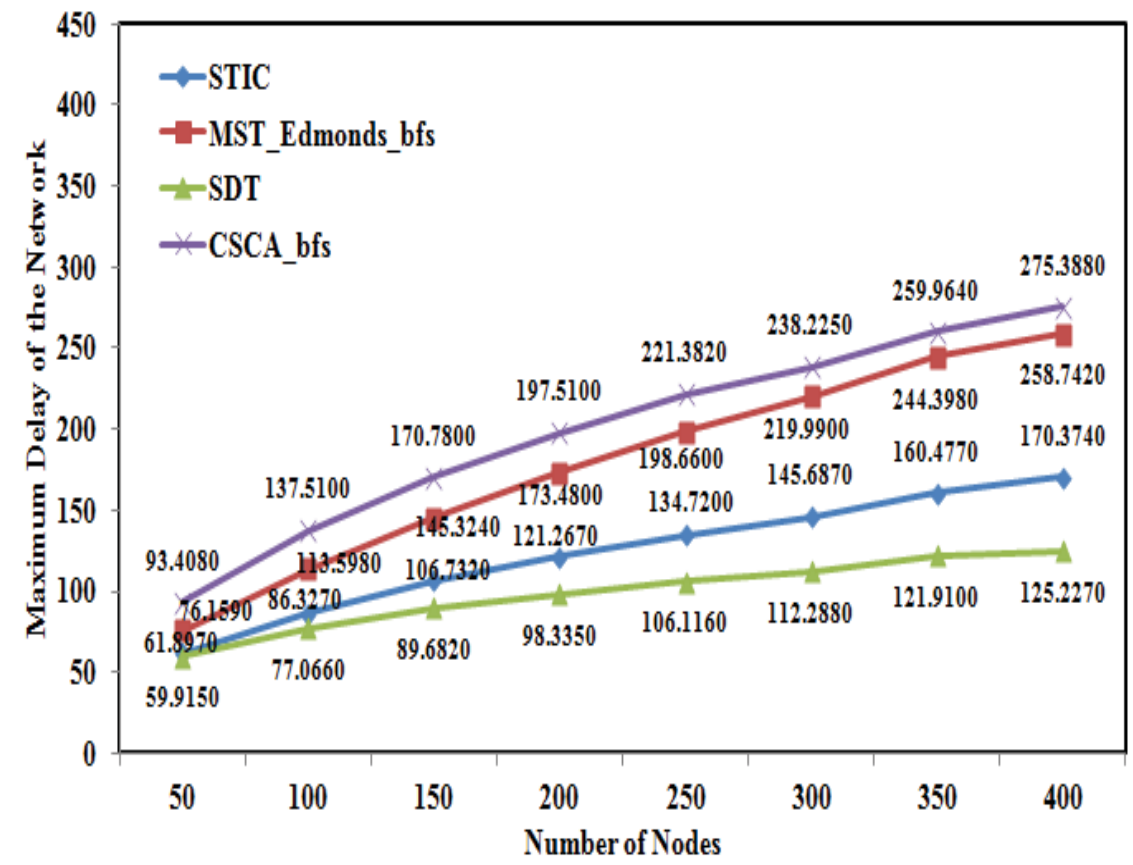

Figure 36: Maximum Delay of the Network at node density 12 for sch_len $=20$

\subsubsection{Average Delay of Broadcast Operation}

As with maximum delay, all versions of the sweep operation increase the average delay for STIC and SDT, while they reduce the average delay for MST_Edmonds and CSCA. At all node densities, SDT obtains the smallest average delay and our algorithm STIC exhibits the nearest performance to it. Although STIC generates less average delay than SDT for smaller values of $N$ at all node densities, its performance degrades with larger values of $N$. At node density 8 MST_Edmonds_bfs exhibits better performance than CSCA_bfs below $N=250$ and then the delay of MST_Edmonds_bfs gradually increases with respect to CSCA_bfs. At node density 10, MST_Edmonds_bfs is doing better than CSCA_bfs below $N=350$ while at node density 12, MST_Edmonds_bfs shows better result for all values of $N$ compared to CSCA_bfs. The average delay of all the algorithms is illustrated in Figures 38 and 39 for sch_len=20. As depicted in Figure 38, at node density 12 for $N=400$, STIC, MST_Edmonds_bfs, CSCA_bfs produces about 21.97\%, $99.94 \%$ and $117.57 \%$ more delay than SDT, respectively. Average delay of all the algorithms with respect to SDT is shown in Table 21 for $N=400$ and sch_len=20. It is noticed SDT performs better than 


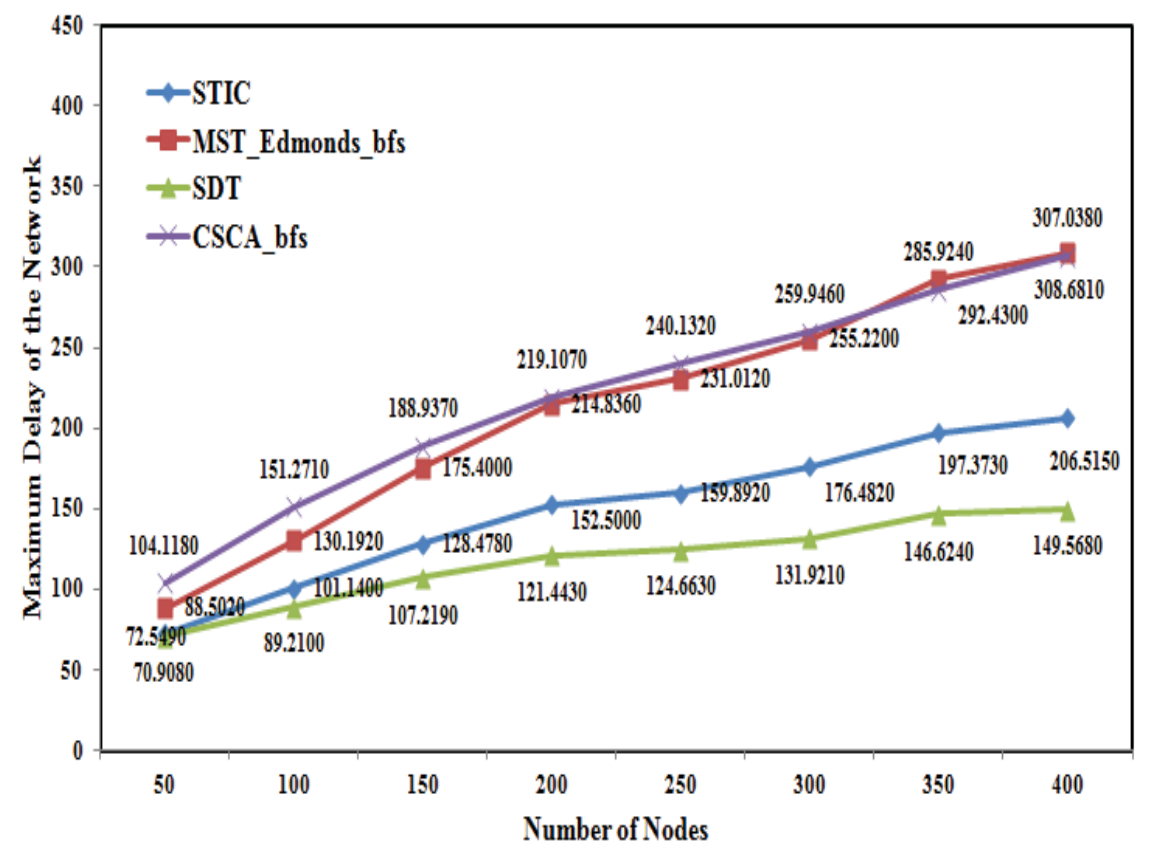

(a) node density $=10$

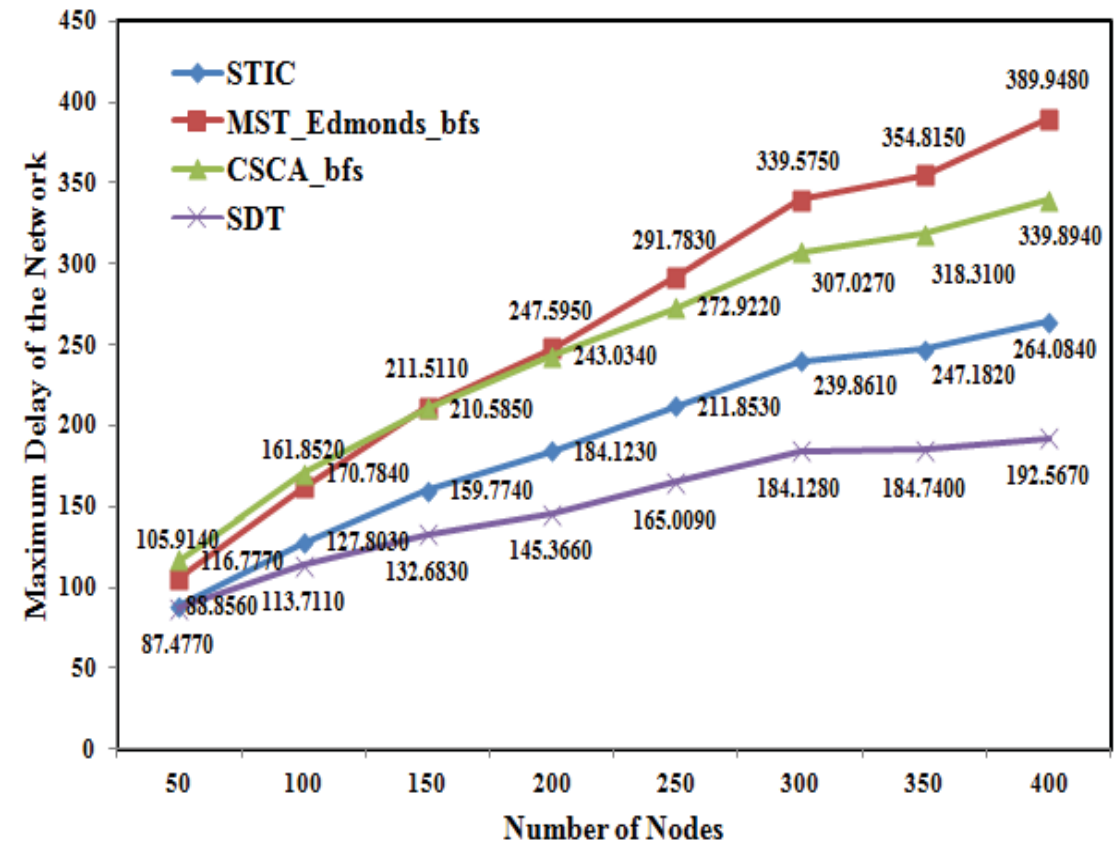

(b) node density $=8$

Figure 37: Maximum Delay of the Network for sch_len $=20$ 




Figure 38: Average Delay of the Network at node density 12 for sch_len $=20$

Table 21: Normalized Average Delay with respect to SDT when $N=400$, sch_len=20

\begin{tabular}{|c|c|c|c|c|}
\hline Node Density & STIC & MST_Edmonds_bfs & CSCA_bfs & SDT \\
\hline \hline 8 & 1.28 & 1.97 & 1.74 & 1.00 \\
\hline 10 & 1.26 & 1.99 & 2.02 & 1.00 \\
\hline 12 & 1.22 & 1.99 & 2.18 & 1.00 \\
\hline
\end{tabular}

other algorithms with increasing values of node density. The sweep operation considerably improves the performance of MST_Edmonds and CSCA compared to SDT. As shown in Table 9, at node density 12 MST_Edmonds produces about $116 \%$ more delay compared to SDT while MST_Edmonds_bfs generates about $99.94 \%$ more delay compared to the same algorithm for $N=400$ as depicted in Table 21. Similarly, CSCA generates about 126\%(as shown in Table 9) more delay compared to SDT while CSCA_bfs generates about 117.57\%(as shown in Table 21) more delay compared to the same algorithm at the same node density for $N=400$. 


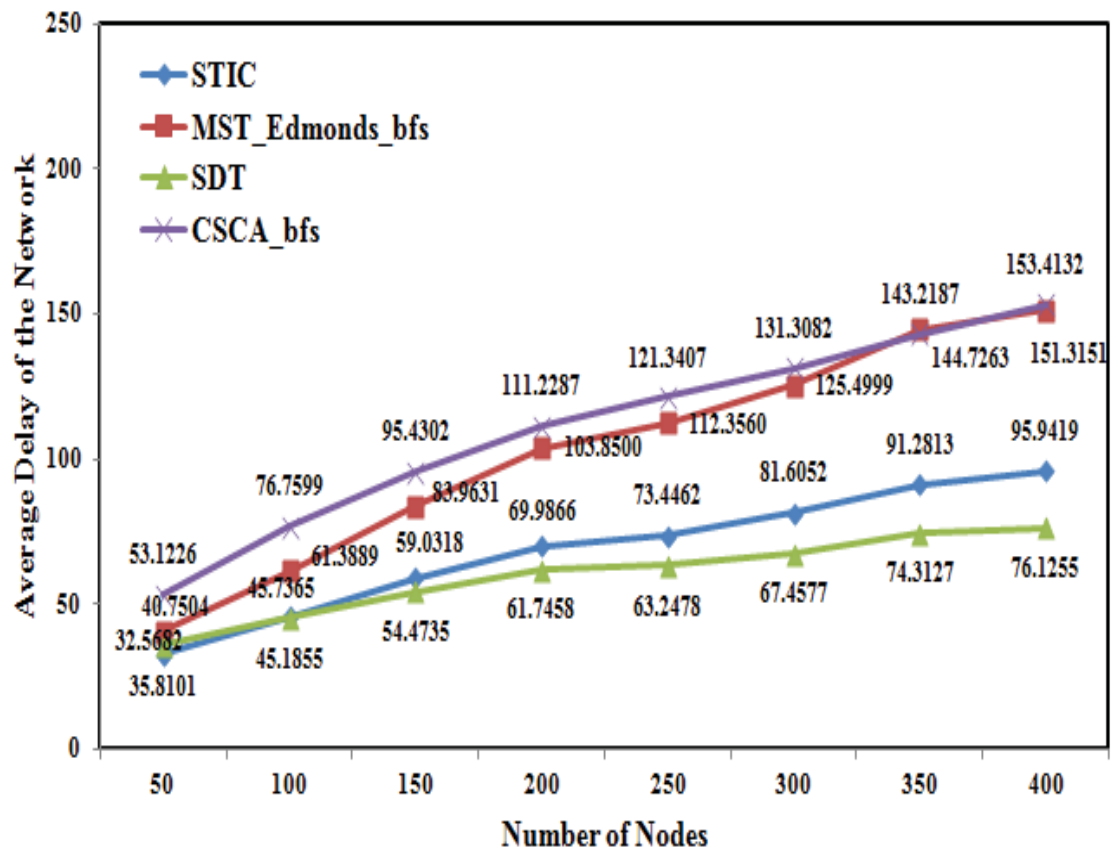

(a) node density $=10$

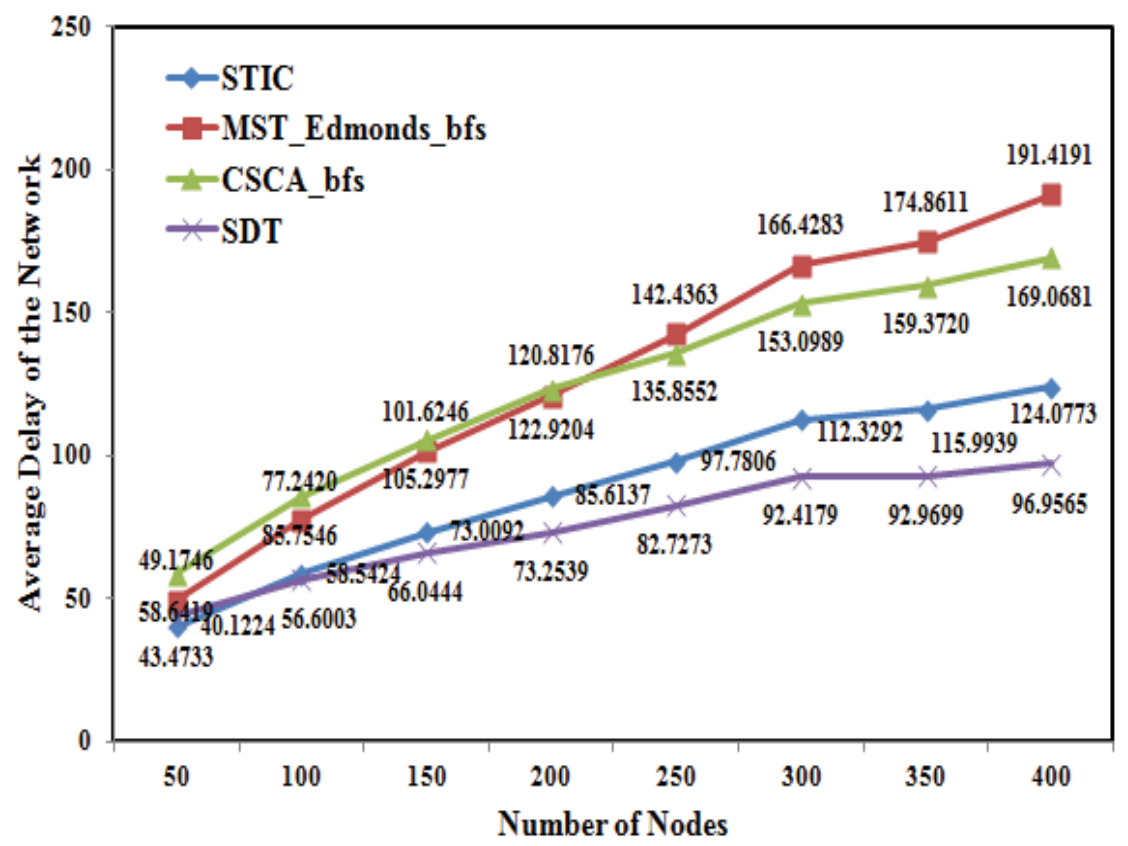

(b) node density $=8$

Figure 39: Average Delay of the Network for sch_len $=20$ 


\subsection{Impact of Node Density}

Node density has a profound impact on all the performance metrics. Given a schedule

length, for all the algorithms we studied, the average additional active time units per node decreases with higher values of node density. As depicted in Table 1, with increasing node densities, not only does MST_Edmonds provides better result, but its difference of performance with other algorithms increases. Similar performance trends are observed for the sweep variants of the algorithms for average additional active time units per node.

Given the schedule length of 15, MST_Edmonds and MST_Edmonds_bfs generate the least number of nodes with active time units 14 in all node densities. With higher values of node density, the performance difference of all the algorithms without sweep operation increases with respect to MST_Edmonds. The performance differences of the sweep variants of all algorithms increases even more compared to MST_Edmonds_bfs with higher node densities except SDT_inc.

The number of node transmissions also decreases with the higher values of node density for all the algorithms. The performance difference of all algorithms with respect to CSCA increases with higher values of node density. Similar patterns of performance are also observed for the sweep variants of the algorithms.

The maximum delay generated by every algorithm reduces as the node density increases. The performance gap of STIC and MSTEEdmonds with SDT reduces while it increases for CSCA with higher node densities. The performance gap of MST_Edmonds_bfs and CSCA_bfs also increases compared to SDT with higher values of node density.

SDT produces minimum average delay at all node densities. The performance gap of STIC with SDT reduces while it increases for CSCA with increasing node densities. MST_Edmonds maintains similar performance gap with respect to SDT at all node densities. The same trends hold for the sweep variants of the algorithms. 


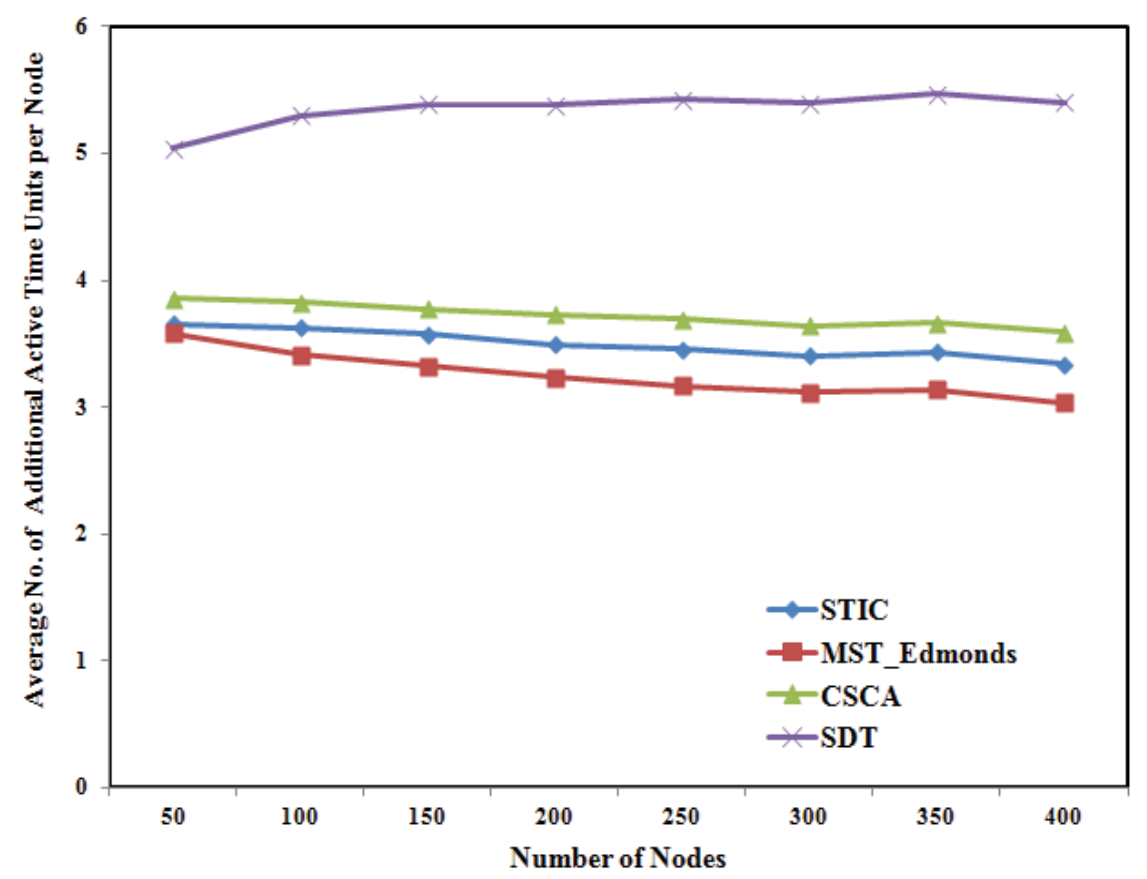

(a) Algorithms without sweep operation at node density 12

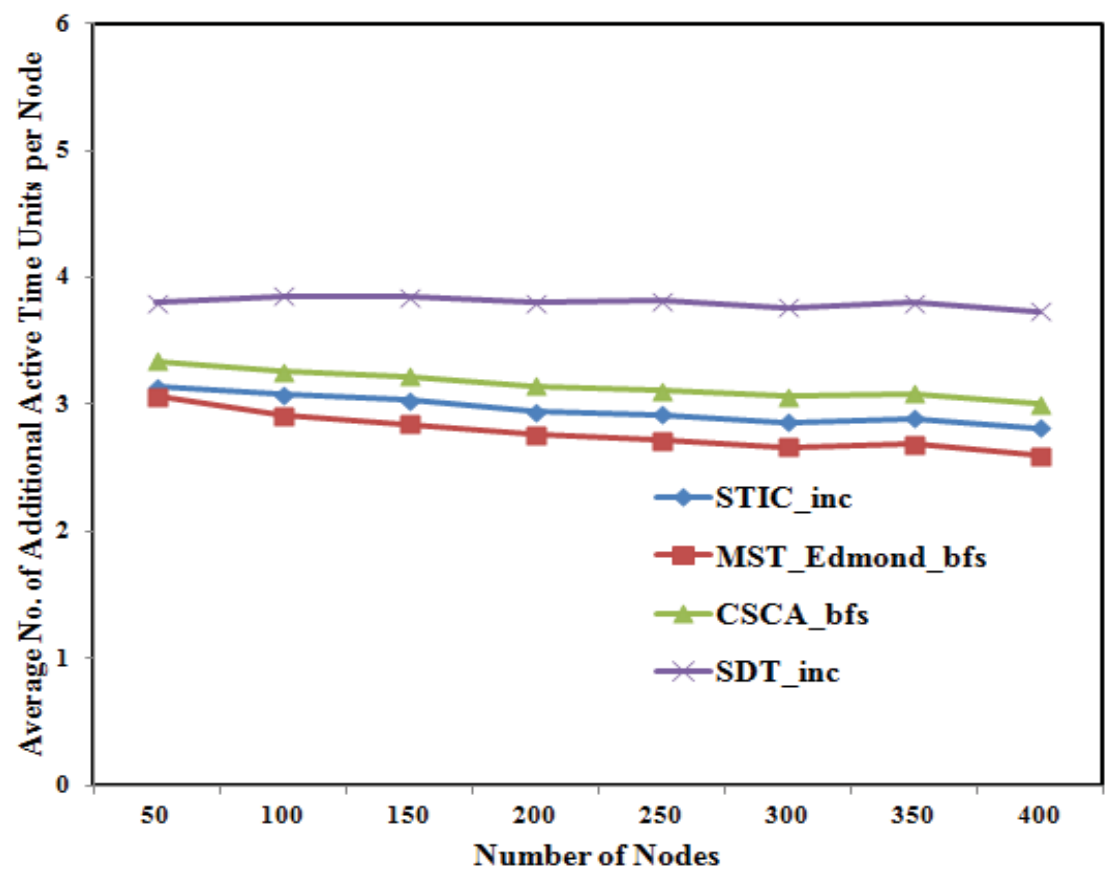

(b) Algorithms with sweep variants at node density 12

Figure 40: Average no. of additional active time units per node for various values of $N$ at node density 12 when sch_len=20 


\subsection{Impact of Number of Nodes}

At every node density, the impact of the number of nodes on additional active time units per node, energy distribution and average node transmissions is small. The average additional active time units for each node decreases slightly with larger values of $N$ for STIC, MST_Edmonds and CSCA at node density 12. As shown in Figure 40(a), SDT starts with a smaller value at $N=50$, then this value increases at $N=100$ after which the value remains the same for higher values of $N$. As depicted in Figure 40(b), similar performance trends are noticed for STIC_inc, MST_Edmonds_bfs and CSCA_bfs. SDT_inc maintains almost the same average additional active time units per node for all values of $N$.

As shown in Figure 41(a), given the sch_len 15, the percentage of nodes with active time units 14 generated by STIC and MST_Edmonds reduces slightly with larger values of $N$. CSCA generates similar percentage of nodes with active time units 14 for all values of $N$. SDT starts with a smaller value at $N=50$ and then increases up to $N=150$ and then maintains almost the same value for other values of $N$. For STIC_inc and MST_Edmonds_bfs, the percentage of nodes with active time units 14 gradually reduces with higher values of $N$ depicted in Figure 41(b). For SDT_inc and CSCA_bfs this percentage slightly decreases at $N=400$ compared to that at $N=50$.

CSCA produces smallest average node transmissions among all the algorithms. As shown in Figure 42(a), at node density 12, average node transmissions for CSCA slightly reduces at $N=400$ compared to that at $N=50$. MST_Edmonds and STIC produce similar values for all $N$. SDT starts with a smaller value at $N=50$ and increases up to $N=150$. It slightly drops at $N=200$ and then maintains almost similar values for remaining $N$. As depicted in Figure 42(b), the average number of node transmissions for CSCA_bfs, MST_Edmonds_bfs and STIC_inc slightly reduces with larger values of $N$. For SDT_inc, average node transmissions starts with a smaller value at $N=50$ and increases upto $N=100$ and then maintains similar result for the remaining values of $N$.

The maximum delay of the broadcast operation increases with larger values of $N$ for all algorithms. SDT generates the minimum value of the maximum delay in all node densities. 


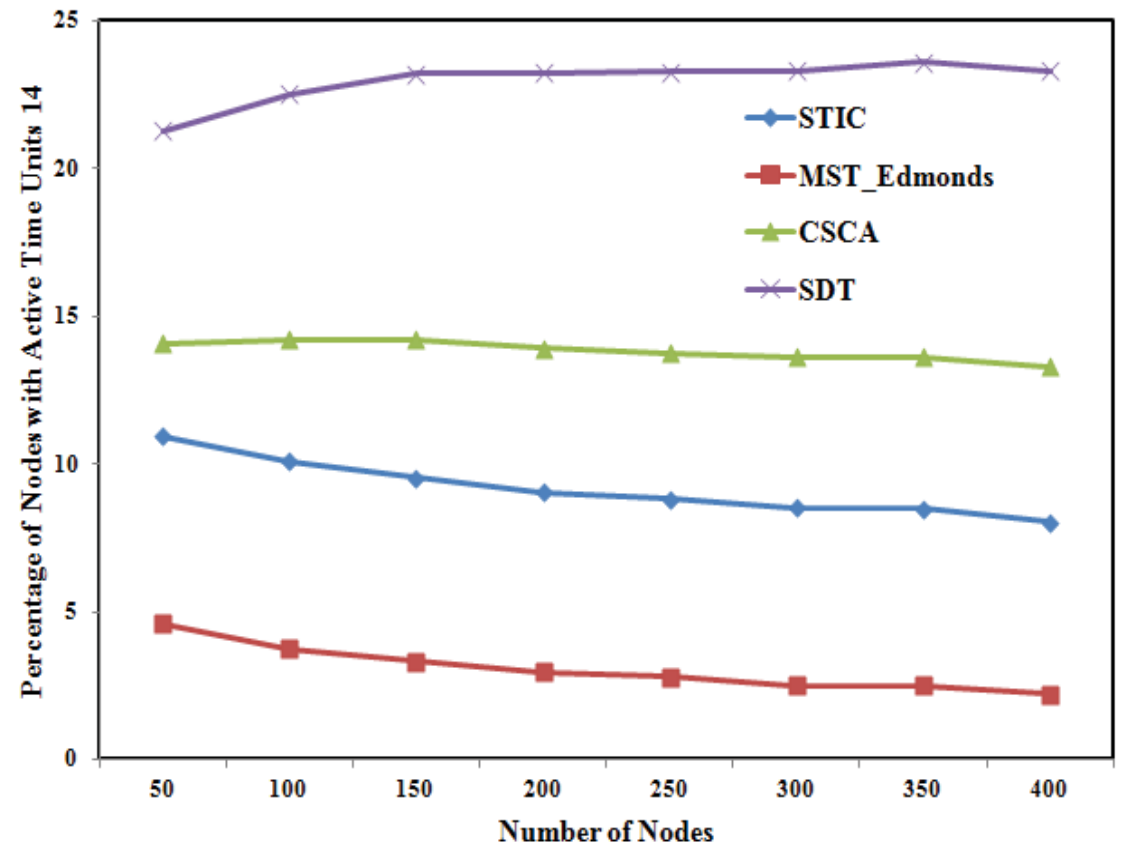

(a) Algorithms without sweep operation at node density 12

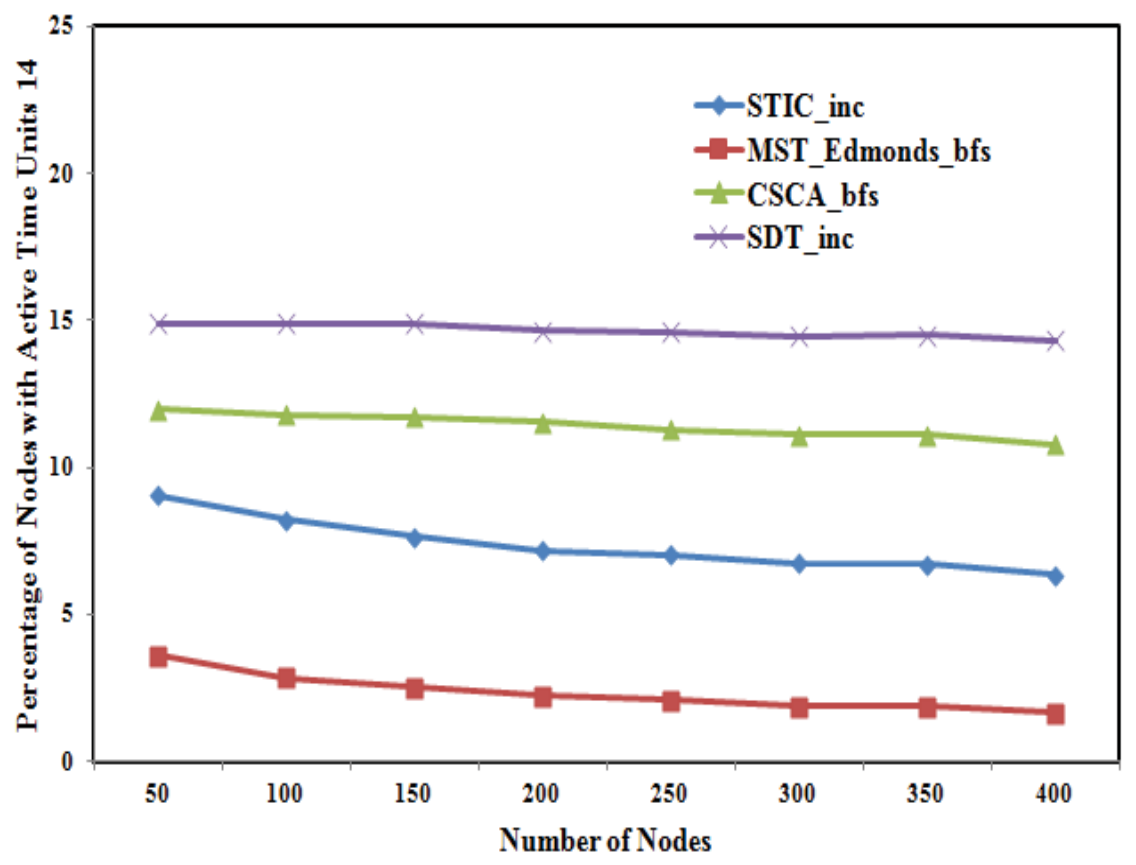

(b) Algorithms with sweep variants at node density 12

Figure 41: Percentage of nodes with active time units 14 for various values of $N$ at node density 12 and sch_len=15 


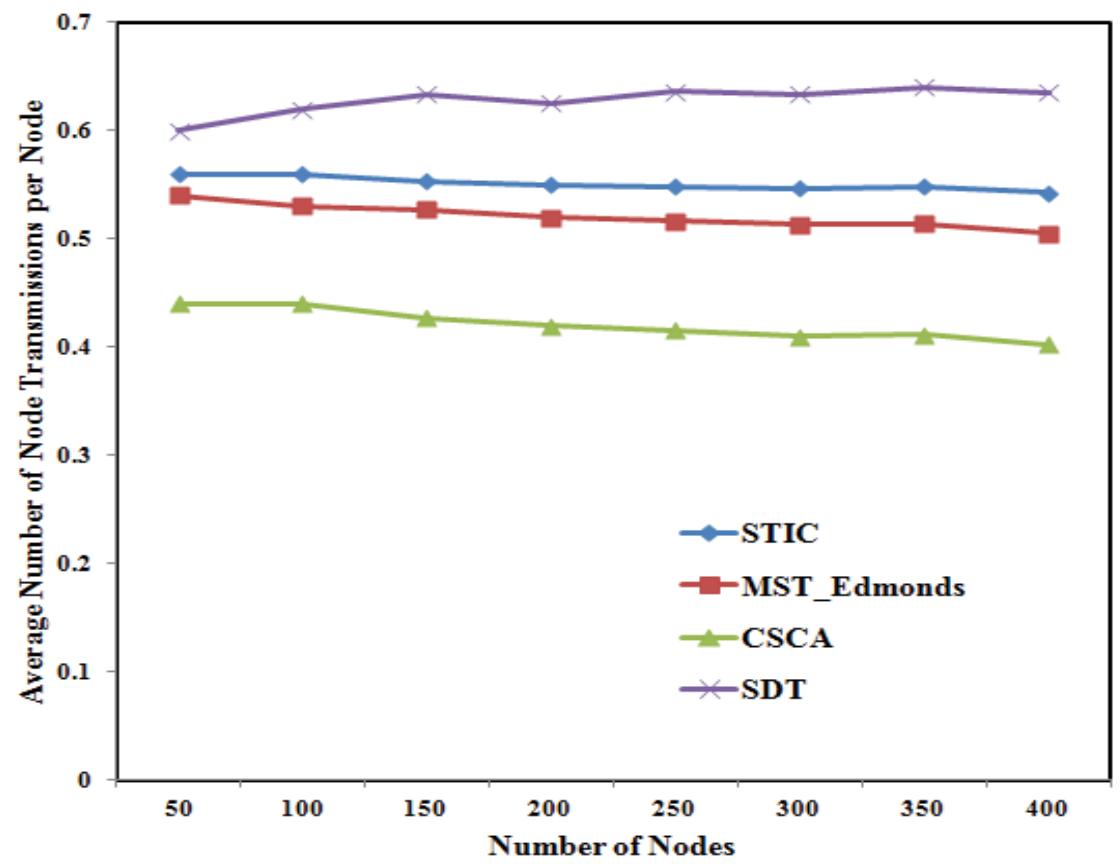

(a) Algorithms without sweep operation at node density 12

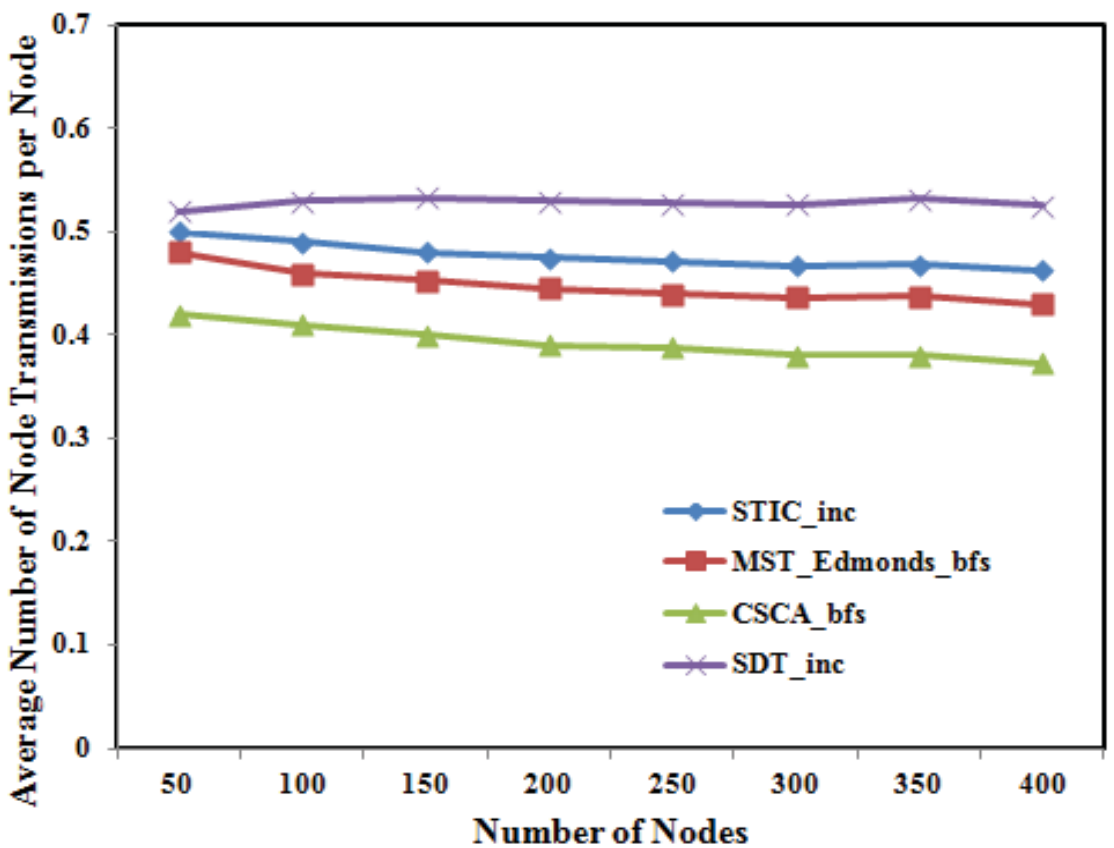

(b) Algorithms with sweep variants at node density 12

Figure 42: Average No. of Node Transmissions for various values of $N$ at node density 12 and sch_len $=20$ 
Our algorithms STIC exhibits the closest performance to it. As shown in Figures 22 and 23 although MST_Edmonds generates smaller delay compared to CSCA at node density 8 and 10 for some values of $N$, it generates better result than the CSCA algorithm for all values of $N$ at node density 12. As illustrated in Figure 36, MST_Edmonds_bfs performs better than CSCA_bfs for node density 12 but for lower densities, it has worse performance than CSCA_bfs for larger values of $N$.

For all algorithms, the average delay of the broadcast operation increases with larger values of $N$. In all node densities, SDT generates the minimum average delay. Our algorithm STIC shows the nearest performance to it. At node density 12, STIC generates smaller delay than SDT up to $N=100$. MST_Edmonds generates better result compared to CSCA for some values of $N$ at node density 8 and 10 and it generates lower delay than CSCA for all values of $N$ at node density 12 as shown in Figure 24. A similar performance trend is also noticed for MST_Edmonds_bfs and CSCA_bfs as shown in Figure 38.

\subsection{Impact of Schedule Length}

Our algorithms reduce the average additional active time units per node for a given value of sch_len. Although the average additional active time units per node per duty cycle is smaller for lower values of sch_len than larger values of sch_len, this does not give an accurate measure of the actual energy consumption with different values of sch_len. Selecting the larger values of sch_len is often advantageous when the algorithm is executing for a longer period of time. We take the least common multiplier of sch_len 5, 10, 15 and 20, which is 60 and determine the total active time units a node needs to be active for a single broadcast within a time period of 60 units if the algorithms work with sch_len 5,10 , 15 and 20. We describe our results at node density 12 for various values of sch_len. As shown in Figure 43, for STIC_inc and $N=400$ a node remains active for a total of 12.5925 time units out of 60 time units for sch_len 5, while it stays awake for only 5.8125 time units out of 60 time units for sch_len 20. Figure 44 shows that with sch_len 5, a node stays awake for 12.5700 time units and 5.5950 time units with sch_len 20 for MST_Edmonds_bfs 


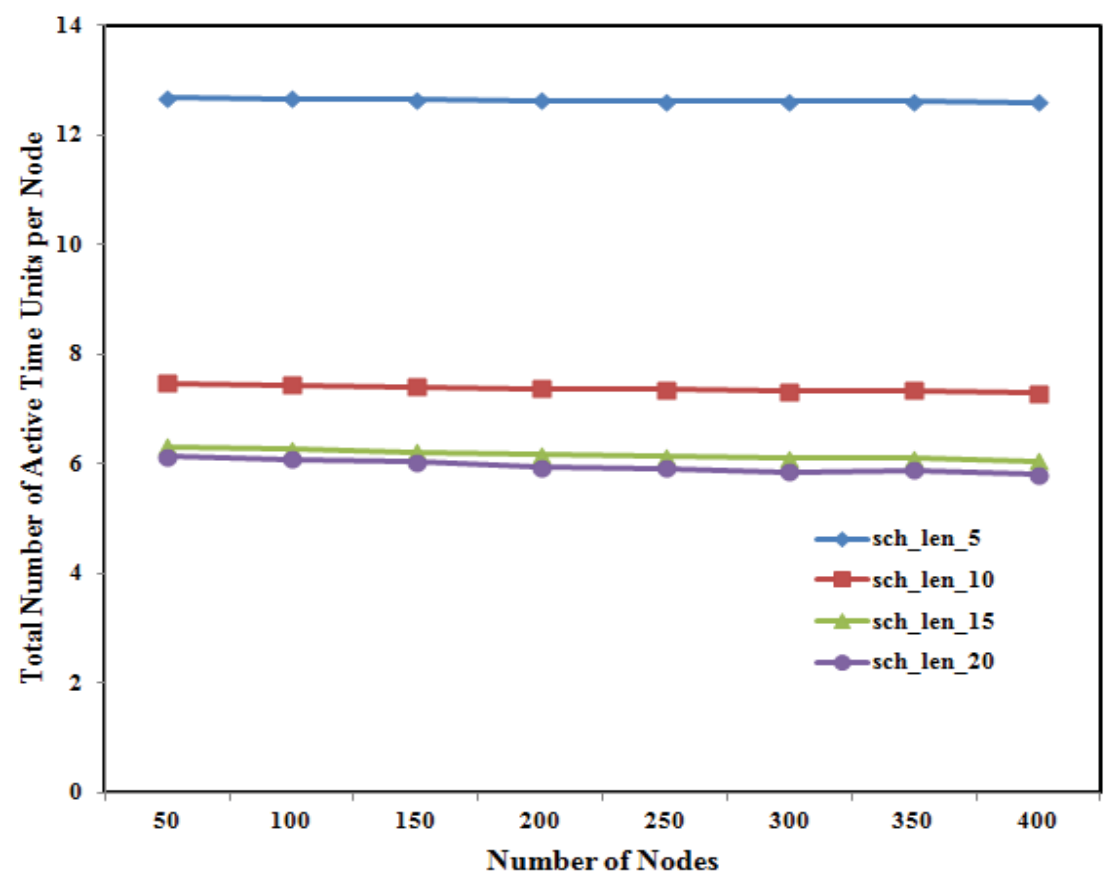

Figure 43: Total Active Time Units per Node for STIC_inc in a time period of 60 at node density $=12$

for the same value of $N$. Clearly it is much more energy-efficient to work with a longer schedule length, if broadcast operations happen infrequently and if a smaller duty cycle is not dictated by other reasons. 


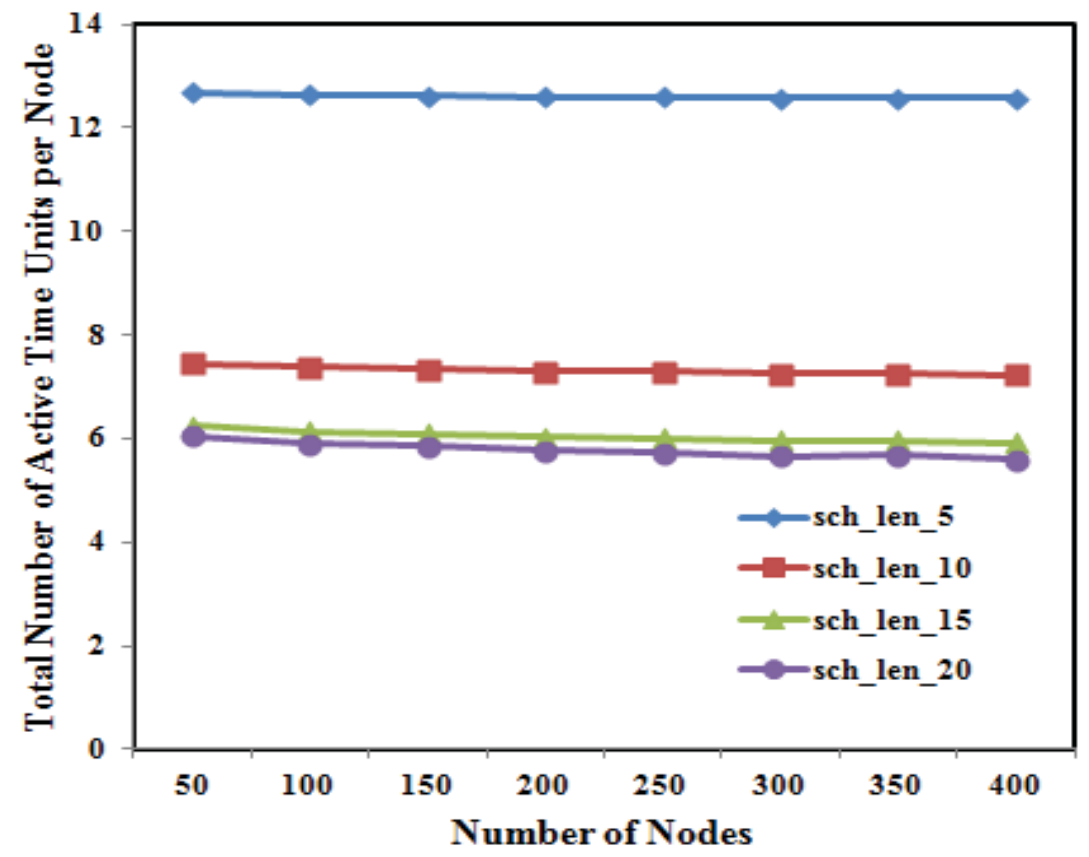

Figure 44: Total Active Time Units per Node for MST_Edmonds_bfs in a time period of 60 at node density $=12$ 


\section{Chapter 5}

\section{Conclusions and Future Work}

In this thesis, we discussed the significance of providing energy efficient broadcast mechanism for duty cycled WSNs in order to ensure the longer network lifetime. The broadcast operation is essential for executing many network activities like routing, topology control, data acquisition, etc. We formulated the problem of constructing an energy efficient broadcast tree for duty cycled WSNs as the MEBT problem and proved that it is NP-hard. We proposed two polynomial time algorithms MST_Edmonds and STIC for constructing an energy-efficient broadcast tree in a duty cycled WSN and then applied several variants of a sweep operation that perform local adjustments on a broadcast tree in order to improve its cost. Simulation results show that our algorithms outperform other previous algorithms in terms of total number of additional active time units and produce the lowest number of highly loaded nodes, with MST_Edmonds being the best of all algorithms. At the same time, they have good performance in terms of maximum and average delay as well as number of node transmissions. MST_Edmonds is better than STIC in terms of the minimum number of transmissions, while the latter algorithm has better delay performance.

The broadcast operation in duty cycled WSNs is highly challenging due to the active/sleep nature of the sensor nodes. Moreover, the problem gets compounded by the unreliable nature of the communication links. We are interested to find broadcast solutions for duty cycled WSNs that will address the unreliable communication links along with a 
mechanism to handle the collisions in order to reduce the number of redundant retransmissions. We proposed two polynomial time heuristic algorithms for the MEBT problem. In future we are interested in finding efficient algorithms with provable performance ratios. Finding a solution for delay efficient broadcasting in duty cycled WSNs is an appealing problem. Adapting the existing broadcast mechanisms of always operational networks for the duty cycled WSNs is also an interesting future research direction. We proposed a centralized solution to construct energy efficient broadcast trees. We can utilize the knowledge of 2-hop neighborhood information of a node to construct distributed and localized algorithms. In this thesis, we construct a broadcast tree with respect to a specific node. However, the construction of a global tree which can be used for broadcast from every node and has acceptable performance is also an attractive problem. Broadcast algorithms that will minimize the maximum energy consumption of every node is also another exciting problem. 


\section{Bibliography}

[1] C. Adjih, P. Jacquet, and L. Viennot. Computing connected dominated sets with multipoint relays. Ad Hoc and Sensor Networks, 1(1-2):27-39, 2005.

[2] I. F. Akyildiz, W. Su, Y. Sankarasubramaniam, and E. Cayirci. A survey on sensor networks. IEEE Communications Magazine, 40(8):102-114, 2002.

[3] I. F. Akyildiz, W. Su, Y. Sankarasubramaniam, and E.Cayirci. Wireless sensor networks: A survey. Computer Networks, 38(2002):393-422, 2002.

[4] J. N. Al-karaki and A. E. Kamal. Routing techniques in wireless sensor networks: A survey. IEEE Wireless Communications, 11(6):6-28, 2004.

[5] N. Aydin, M. Karaca, and O. Ercetin. Energy-optimal scheduling in low duty cycle sensor networks. In SPECTS, 2011: Proceedings of the International Symposium on Performance Evaluation of Computer and TeleCommunication Systems, pages 119$126,2011$.

[6] J. Cartigny, F. Ingelrest, D. Simplot-Ryl, and I. Stojmenovic. Localized lmst and rng based minimum-energy broadcast protocols in ad hoc networks. Ad Hoc Networks, 3(1):1-16, 2005.

[7] J. Cartigny, D. Simplot, and I. Stojmenovic. Localized minimum-energy broadcasting in ad-hoc networks. In IEEEINFOCOM2003: Proceedings of the 22nd Annual Joint Conference of the IEEE Computer and Communications Societies, pages 2210-2217, 2003. 
[8] X. Chen, M. Faloutsos, and S. V. Krishnamurthy. Power adaptive broadcasting with local information in ad hoc networks. In ICNP2003: Proceedings of the 11th IEEE International Conference on Network Protocols, pages 168-178, 2003.

[9] C.-F. Chiasserini and M. Garetto. Modeling the performance of wireless sensor networks. In INFOCOM'04: Proceedings of the 23rd Conference of the IEEE Communications Society, pages 220-231, 2004.

[10] J. Edmonds. Optimum branchings. Journal of Research of the National Bureau of Standards, 71B:233-240, 1967.

[11] Y. Gu and T. He. Data forwarding in extremely low duty-cycle sensor networks with unreliable communication links. In SenSys'0\%: Proceedings of the 5th International Conference on Embedded Networked Sensor Systems, pages 321-334, 2007.

[12] S. Guo, Y. Gu, B. Jiang, and T. He. Opportunistic flooding in low-duty cycle wireless sensor networks with unreliable link. In MOBICOM'09: Proceedings of the 15th Annual International Conference on Mobile Computing and Networking, pages 133-144, 2009.

[13] Z. J. Haas, J. Y. Halpern, and L. Li. Gossip-based ad hoc routing. In INFOCOM'02: Proceedings of Twenty-First Annual Joint Conference of IEEE Computer and Communications Societies, pages 1707-1716, 2002.

[14] Z. J. Haas and M. Nikolov. Towards optimal broadcast in wireless networks. In MSWIM'11: Proceedings of the 14th ACM International Conference on Modeling, Analysis and Simulation of Wireless and Mobile Systems, pages 213-222, 2011.

[15] W. Heinzelman, A. Chandrakasan, and H. Balakrishnan. Energy-efficient communication protocol for wireless microsensor networks. In Proceedings of the 33rd Hawaii International Conference on System Sciences - 2000, pages 1-10, 2000.

[16] W. Heinzelman, J. Kulik, and H. Balakrishnan. Adaptive protocols for information dissemination in wireless sensor networks. In MOBICOM,1999: Proceedings of the 
5th annual ACM/IEEE international conference on Mobile computing and networking, pages 174-185, 1999.

[17] J. Hong, J. Cao, W. Li, S. Lu, and D. Chen. Sleeping schedule-aware minimum latency broadcast in wireless ad hoc networks. In ICC, 2009: Proceedings of the IEEE International Conference on Communications, pages 1-5, 2009.

[18] J. Hong, J. Cao, W. Li, S. Lu, and D. Chen. Minimum-transmission broadcast in uncoordinated duty-cycled wireless ad hoc networks. IEEE Transaction on Vehicular technology, 59(1):307-318, 2010.

[19] F. Ingelrest and D. Simplot-Ryl. Localized broadcast incremental power protocol for wireless ad hoc networks. In ISCC2005: Proceedings of the IEEE Symposium on Computers and Communication, pages 28-33, 2005.

[20] F. Ingelrest, D. Simplot-Ryl, and I. Stojmenovic. Optimal transmission radius for energy efficient broadcasting protocols in ad hoc and sensor networks. IEEE Transactions on Parallel and Distributed Systems, 17(6):536-547, 2006.

[21] C. Intanagonwiwat, R. Govindan, and D. Estrin. Directed diffusion: a scalable and robust communication paradigm for sensor networks. In MOBICOM,2000: Proceedings of the 6th annual international conference on Mobile computing and networking, pages $56-67,2000$.

[22] X. Jiao, W. Lou, J. Ma, J. Cao, X. Wang, and X. Zhou. Minimum latency broadcast scheduling in duty-cycled multihop wireless networks. IEEE Transactions on Parallel and Distributed Systems, 23(1):110-117, 2012.

[23] R. Jurdak, A. G. Ruzzelli, and G.M.P. OHare. Radio sleep mode optimization in wireless sensor networks. IEEE Transactions on Mobile Computing, 9(7):955-968, 2010.

[24] A. Keshavarzian, H. Lee, and L. Venkatraman. Wakeup scheduling in wireless sensor networks. In MOBIHOC,2006: Proceedings of the 7th ACM international symposium on Mobile ad hoc networking and computing, pages 322-333, 2006. 
[25] P. Kysdsnur, R.R. Choudhury, and I. Gupta. Smart gossip: An adaptive gossipbased broadcasting service for sensor networks. In Proceedings of IEEE International Conference on Mobile Ad Hoc and Sensor Systems, pages 91-100, 2006.

[26] P. Levis, N. Patel, D. Culler, and S. Shenker. Trickle: A self-regulating algorithm for code propagation and maintenance in wireless sensor networks. In NSDI'04: Proceedings of the First USENIX/ACM Symposium on Networked Systems Design and Implementation, pages 15-28, 2004.

[27] N. Li and J. C. Hou. BLMST: A scalable, power-efficient broadcast algorithm for wireless networks. In QSHINE2004: Proceedings of the 1st International Conference on Quality of Service in Heterogeneous Wired/Wireless Networks, 2004.

[28] W. Liang. Constructing minimum-energy broadcast trees in wireless ad hoc networks. In MOBIHOC'02: Proceedings of the Third ACM International Symposium on Mobile Ad Hoc Networking and Computing, pages 112-122, 2002.

[29] H. Lim and C. Kim. Multicast tree construction and flooding in wireless ad hoc networks. In Proceedings of ACM International Workshop Modeling, Analysis and Simulation of Wireless and Mobile Systems, pages 61-68, 2000.

[30] W. Lou and J. Wu. On reducing broadcast redundancy in ad hoc wireless networks. IEEE Transactions on Mobile Computing, 1(2):111-122, 2002.

[31] G. Lu, N. Sadagopan, B. Krishnamachari, and A. Goel. Delay efficient sleep scheduling in wireless sensor networks. In INFOCOM'05: Proceedings of the 24th Joint Conference of the IEEE Computer and Communications Societies, pages 2470-2481, 2005.

[32] S.-Y. Ni, Y.-C.Tseng, Y.-S. Chen, and J.-P. Sheu. The broadcast storm problem in a mobile ad hoc network. In MOBICOM'99:Proceedings of International Conference on Mobile Computing and Networks, pages 151-162, 1999.

[33] J. Park and S. Sahani. Maximum lifetime broadcasting in wireless networks. IEEE Transactions on Computers, 54(9):1081-1089, 2005. 
[34] W. Peng and X-C. Lu. On the reduction of broadcast redundancy in mobile ad hoc networks. In MOBIHOC,2000: Proceedings of the 1st Annual Workshop on Mobile and Ad Hoc Networking and Computing, pages 129-130, 2000.

[35] R. C. Prim. Shortest connection networks and some generalizations. Bell System Technical Journal, 36:1389-1401, 1957.

[36] A. Qayyum, L. Viennot, and A. Laouiti. Multipoint relaying for flooding broadcast messages in mobile wireless networks. In HICSS-35'02: Proceedings of the 35th Hawaii International Conference on System Sciences, pages 298-307, 2002.

[37] A. Qayyum, L. Viennot, and A. Laouiti. An enhanced approach to determine a small forward node set based on multipoint relays. In IEEEVTCH2003: Proceedings of the of IEEE Vehicular Technology Conferenece, pages 2774-2777, 2003.

[38] A. G. Ruzzelli, P. Cotan, G. M. P. OHare, R. Tynan, and P. J. M havinga. Protocol assessment issues in low duty cycle sensor networks: The switching energy. In SUTC'06: Proceedings of IEEE International Conference on Sensor Networks, Ubiquitous and Trustworthy Computing, pages 101-108, 2006.

[39] K. Selvakumar and S. Nithya. d2-coloring of a graph. The Journal of Mathematics and Computer Science, 3(2):102-111, 2011.

[40] F. Stann, J. Heidemann, R. Shroff, and M. Z. Murtaza. RBP: Robust broadcast propagaion in wireless networks. In SenSys2006: Proceedings of the 4 th International Conference on Embedded Networked Sensor Systems, pages 58-98, 2006.

[41] I. Stojmenovic. Comments and corrections to dominating sets and neighbor elimination-based broadcasting algorithms in wireless networks. IEEE Transactions on Parallel and Distributed Systems, 15(11):1054-1055, 2004.

[42] I. Stojmenovic, M. Seddigh, and J. Zunic. Dominating sets and neighbor elimination based broadcasting algorithms in wireless networks. IEEE Transactions on Parallel and Distributed Systems, 13(1):14-25, 2002. 
[43] Y. Sun, O. Gurewitz, S. Du, L. Tang, and D. B. Johnson. ADB: An efficient multihop broadcast protocol based on asynchronous duty-cycling in wireless sensor networks. In SenSys'09: Proceedings of the 7th ACM Conference on Embedded Networked Sensor Systems, pages 43-56, 2009.

[44] Y. C. Tseng, S. Y. Ni, and E. Y. Shih. Adaptive approaches for relieving broadcast storms in wireless multihop mobile ad hoc networks. IEEE Transactions on Computers, $52(5): 545-557,2003$.

[45] F. Wang and J. Liu. RBS: A reliable broadcast service for large-scale low duty-cycled wireless sensor networks. In ICC'08: Proceedings of IEEE International Conference on Communications, pages 2416-2420, 2008.

[46] F. Wang and J. Liu. Duty-cycle-aware broadcast in wireless sensor networks. In INFOCOM'09: Proceedings of the 28th Conference on Computer Communications, pages 468-476, 2009.

[47] W. Wang and B. Soong. Collision-free and low-latency scheduling algorithm for broadcast operation in wireless ad hoc networks. IEEE Communications Letters, 11(10):1-3, 2007.

[48] P. Wei and L. Xicheng. AHBP: An efficient broadcast protocol for mobile ad hoc networks. Journal of Computer Science and Technology, 16(2):114-125, 2001.

[49] J. Wieselthier, G. Nguyen, and A. Ephremides. On the construction of energy-efficient broadcast and multicast trees in wireless sensor networks. In IEEE INFOCOM 2000: Proceedings of the 19th Annual Joint Conference of the IEEE Computer and Communications Societies, pages 585-594, 2000.

[50] J. E. Wieselthier, G. D. Nguyen, and A. Ephremides. Distributed algorithms for energyefficient broadcasting in ad hoc networks. In MILCOM,2002: Proceedings of the Military Communications Conference, pages 820-825, 2002. 
[51] J. E. Wieselthier, G. D. Nguyen, and A. Ephremides. Energy-efficient broadcast and multicast trees for wireless networks. Mobile Networks and Applications, 7(6):481-492, 2002.

[52] J. Wu and H. Li. On calculating connected dominating set for efficient routing in ad hoc wireless networks. In DlALM1999: Proceedings of the 3rd International Workshop on Discrete Algorithms and Methods for Mobile Computing and Communications, pages 7-14, 1999.

[53] J. Wu, W. Lou, and F. Dai. Extended multipoint relays to determine connected dominating sets in manets. IEEE Transactions on Computers, 55(3):334-347, 2006. 